\title{
Effects of dapagliflozin and combination therapy with exenatide on food-cue induced brain activation in patients with type 2 diabetes: a randomized controlled trial
}

\author{
Lotje van Ruiten', Dick Veltman², Max Nieuwdorp ${ }^{3}$, Richard IJzerman' \\ ${ }^{1}$ Diabetes Center, Department of Internal Medicine, Amsterdam UMC, location Vumc, Amsterdam, The Netherlands; ${ }^{2}$ Department of Psychiatry, \\ Amsterdam UMC, location Vumc, Amsterdam, The Netherlands; ${ }^{3}$ Department of Experimental Vascular Medicine, Amsterdam UMC, Location AMC, \\ Amsterdam, The Netherlands \\ E-mail: c.ruiten@amsterdamumc.nl
}

\section{BACKGROUND}

Sodium-glucose cotransporter-2 inhibitors (SGLT2i) cause less weight loss than expected based on urinary calorie excretion. This may be explained by SGLT2i-induced alterations in central reward and satiety circuits, leading to increased appetite and food intake. Glucagon-like peptide-1 receptor agonists are associated with reduced appetite and body weight, mediated by direct and indirect CNS effects. We investigated the separate and combined effects of dapagliflozin and exenatide on the CNS in obese type-2 diabetes subjects.

\section{METHODS}

This was a 16-week, double-blind, randomized, placebo-controlled trial. Obese patients with type- 2 diabetes were randomized (1:1:1:1) to dapagliflozin $10 \mathrm{mg}$ with exenatide-matched placebo, exenatide twice-daily $10 \mu \mathrm{g}$ with dapagliflozin-matched placebo, dapagliflozin and exenatide, or double placebo $(\mathrm{n}=64$, age $63.5 \pm 0.9$ years, BMI $31.7 \pm 0.6 \mathrm{~kg} / \mathrm{m}^{2}$ ). Using functional MRI, the effects of treatments on CNS responses to viewing food pictures were assessed after 10 days and 16 weeks of treatment.
Dapagliflozin increased carbohydrate intake $(21.3 \pm 9.6$ grams, $\mathrm{p}=0.029)$. After short-term treatment, dapagliflozin increased $(\mathrm{T}=3.4, \mathrm{p}=0.014)$, whereas exenatide decreased $(\mathrm{T}=3.6, \mathrm{p}=0.011) \mathrm{CNS}$ activation in left putamen. Combination therapy had no effect on responses to food pictures. After longer-term treatment, no changes in CNS activation were observed with dapagliflozin. CNS activation was reduced with exenatide in bilateral insula (right, $\mathrm{T}$ $=4.3, \mathrm{p}=0.003$; left, $\mathrm{T}=3.8, \mathrm{p}=0.016$ ), and with dapagliflozin-exenatide in right amygdala $(\mathrm{T}=3.0, \mathrm{p}=0.032)$.

\section{CONCLUSION}

The discrepancy between observed and expected weight loss with SLGT2i treatment may be explained by changes in appetite and dietary intake due to increased activation in feeding regulation areas in the CNS in response to food stimuli. Exenatide mitigated the dapagliflozin-induced increase in CNS activation, but the exenatide-reducing effect was not as strong as with exenatide monotherapy, partially explaining why the observed weight loss with dapagliflozin-exenatide is not fully additive.

\section{RESULTS}

Weight and HbAlc reduction were largest in the combination group $(-2.8 \pm 0.5 \mathrm{~kg}, \mathrm{p}<0.001 ;-1.2 \pm 0.19 \%, \mathrm{p}<0.001)$.

\section{2}

\section{Cost-effectiveness and cost-utility of foot temperature monitoring for preventing diabetic foot ulcer recurrence: a randomized controlled trial}

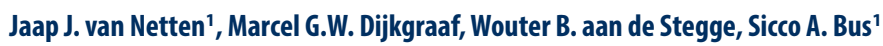

\author{
${ }^{1}$ Amsterdam UMC, University of Amsterdam, Department of Rehabilitation, Amsterdam Movement Sciences, Amsterdam, The Netherlands \\ E-mail:j.j.vannetten@amsterdamumc.nl
}

\section{BACKGROUND}

The skin of people with diabetic foot disease is thought to heat up from weight-bearing activity before it breaks down into ulceration. We assessed cost-effectiveness and cost-utility of at-home foot skin temperature monitoring for the prevention of diabetic foot ulcer recurrence. 
In this outcome-assessor-blinded multicenter RCT, we randomly assigned people at high risk of ulceration to usual care (i.e. podiatric treatment, education, and therapeutic footwear) or usual care plus measuring skin temperatures at 6-8 plantar sites per foot each day (enhanced therapy). Foot care costs from a societal perspective were obtained via questionnaires and electronic health records. Utilities were calculated based on EQ-5D-3L scores. Primary outcome for effectiveness was foot ulcer recurrence in 18 months. Group differences were assessed by calculating $95 \%$ confidence intervals, corrected for bias with accelerated non-parametric bootstrapping. Incremental cost-effectiveness ratios (ICER) and incremental cost-utility ratios (ICUR) were calculated as the ratio between costs and effect/utility differences between enhanced therapy and usual care.

\section{RESULTS}

Total foot care costs per participant during 18 months follow-up were lower in enhanced therapy $(n=151$; $€ 6,067$
(SD: $€ 13,778)$ ) compared to usual care ( $\mathrm{n}=153$; $€ 7,376$ (SD: $€ 15,790) ; \mathrm{p}=0.450$ ). Enhanced therapy was cheaper and more effective in $78 \%$ of the cost-effect pairs. Enhanced therapy had $79 \%$ probability of being cost-effective at a willingness-to-pay of $€ 0$ per participant who remains ulcer-free. Quality-adjusted life years were lower in enhanced therapy (1.085 (SD: 0.33)) than in usual care (1.119 (SD: 0.31$) ; p=0.348)$. Enhanced therapy was cheaper but with lower quality of life in $68 \%$ of cost-utility pairs. Enhanced therapy had $45 \%$ probability of achieving cost-utility at a willingness-to-pay of $€ 50,000$ per QALY gained.

\section{CONCLUSION}

In this first-ever societal-perspective cost-effectiveness RCT in the field of diabetic foot disease, we found temperature monitoring cost-effective in foot ulcer prevention, at the expense of lower quality of life.

\section{3}

\section{Androgens and Development of Post-Transplantation Diabetes Mellitus in Male Kidney Transplant Recipients: A post-hoc analysis of a prospective study}

Suzanne P. Stam ${ }^{1}$, Sara Sokooti ${ }^{1}$, Michele F. Eisenga ${ }^{1}$, Anna van der Veen ${ }^{2}$, Antonio W. Gomes-Neto ${ }^{1}$, Peter R. van Dijk ${ }^{3}$, Jelmer J. van Zanden ${ }^{4}$, Michel J. Vos ${ }^{2}$, Ido P. Kema², André P. van Beek ${ }^{3}$, Stephan J. Bakker ${ }^{1}$, and TransplantLines Investigators ${ }^{5}$

${ }^{1}$ Department of Internal Medicine, Division of Nephrology, University of Groningen, University Medical Center Groningen, Groningen, The Netherlands; ${ }^{5}$ Groningen Transplant Center, University of Groningen, University Medical Center Groningen, Groningen, The Netherlands

E-mail:s.p.stam@umcg.nl

\section{BACKGROUND}

Post-transplantation Diabetes Mellitus (PTDM) effects up to $30 \%$ of all kidney transplant recipients (KTR). Recent studies in mice found that sufficient androgen levels are necessary for $\beta$-cell health and adequate insulin secretion. This raises the question whether a similar relationship might be present in KTR. Hence, we hypothesized that dihydrotestosterone and testosterone are associated with the development of PTDM in male KTR.

\section{METHODS}

We conducted a post-hoc analyses of a prospective single-center cohort study including adult male KTR with a functioning graft $\geq 1$ year post-transplantation. Androgen levels were assessed by liquid chromatography tandem mass spectrometry and the development of PTDM was defined according to the American Diabetes Association's criteria.

\section{RESULTS}

We included 243 male KTR (age $51 \pm 14$ years), with a median dihydrotestosterone $0.9[0.7-1.3] \mathrm{nmol} / \mathrm{L}$ and testosterone of 12.1 [9.4-15.8] nmol/L. During 5.3 [3.7-5.8] years of follow-up, 28 KTR (11.5\%) developed PTDM. A clear association was observed as 15 (19\%), 10 (12\%), and 3 (4\%) male KTR developed PTDM in the respective tertiles of dihydrotestosterone $(\mathrm{p}=0.008)$. In Cox regression analyses, both dihydrotestosterone and testosterone as continuous variables, were inversely associated with the risk to development PTDM, independent of glucose and HbA1c (HR 0.31; 95\%CI [0.16-0.59], p < 0.001 and HR $0.32 ; 95 \% \mathrm{CI}[0.15-0.68], \mathrm{p}=0.003$ respectively).

\section{CONCLUSION}

Our results suggest that low androgen levels are a novel potential modifiable risk factor for the development of PTDM in male KTR. 


\title{
A single hypoglycaemic event drives a prolonged inflammatory response in individuals with and without type 1 or type 2 diabetes
}

\author{
Clementine E.M. Verhulst ${ }^{1}$, Therese W. Fabricius ${ }^{2}$, Julia I.P. van Heck ${ }^{1}$, Rinke Stienstra ${ }^{1,3}$, Cees J. Tack ${ }^{1}$, Ulrik Pedersen-Bjergaard ${ }^{2,4}$, Bastiaan E. de Galan ${ }^{1,5}$ \\ on behalf of the hypo-RESOLVE consortium \\ ${ }^{1}$ Department of Internal Medicine, Radboudumc, Nijmegen, The Netherlands; ${ }^{2}$ Department of Endocrinology and Nephrology, Nordsjollands Hospital, \\ Hillerød, Denmark; ${ }^{3}$ Department of Nutrition, Metabolism and Genomics Group, Division of Human Nutrition and Health, Wageningen University, \\ Wageningen, The Netherlands; ${ }^{4}$ Department of Clinical Medicine, Faculty of Health and Medical Sciences, University of Copenhagen, Denmark; \\ ${ }^{5}$ Department of Internal Medicine, Maastricht University Medical Centre, MUMC+ Maastricht, The Netherlands \\ E-mail: clementine.verhulst@radboudumc.nl
}

\section{BACKGROUND}

Iatrogenic hypoglycaemia acutely activates the immune system and is associated with an increased risk for atherosclerotic disease development during diabetes. Since atherosclerosis is driven by vascular inflammation, we set out to determine the duration of the pro-inflammatory response to a hypoglycaemic event in people with and without diabetes.

\section{METHODS}

Adults with type 1 diabetes $(\mathrm{n}=47)$ or with insulin-treated type 2 diabetes $(n=15)$, and age-matched controls without diabetes $(n=32)$ were recruited to undergo a hyperinsulinaemic-normoglycaemic $(5.21 \pm 0.40 \mathrm{mmol} / \mathrm{L})$-hypoglycaemic $(2.78 \pm 0.13 \mathrm{mmol} / \mathrm{L})$ glucose clamp. During normoglycaemia and hypoglycaemia, and 24 hours, 72 hours and one week later blood was drawn to determine immune cell composition and circulating concentrations of hs-CRP.

\section{RESULTS}

Acute hypoglycaemia increased counts of granulocytes $\left(3.81 \pm 1.44\right.$ vs $\left.4.64 \pm 1.94 \cdot 10^{3} / \mu \mathrm{L}\right)$, lymphocytes $(1.57 \pm$
0.53 vs $\left.2.69 \pm 0.95 \cdot 10^{3} / \mu \mathrm{L}\right)$ and monocytes $(0.40 \pm 0.14$ vs $\left.0.62 \pm 0.22 \cdot 10^{3} / \mu \mathrm{L}\right)$ in whole group (all $\left.\mathrm{p}<0.001\right)$. Granulocyte counts fell below normoglycaemic levels after 72 hours $\left(3.28 \pm 1.33 \cdot 10^{3} / \mu \mathrm{L}, \mathrm{p}<0.001\right)$ and remained suppressed thereafter $\left(3.41 \pm 1.50 \cdot 10^{3} / \mu \mathrm{L}, \mathrm{p}=0.024\right)$, whereas lymphocyte and monocyte counts remained elevated till one week after the hypoglycaemic event (both $\mathrm{p}<0.001$ ) in the whole group. Hs-CRP concentrations increased from $2.07 \pm 3.20 \mu \mathrm{g} / \mathrm{mL}$ during normoglycaemia to $2.71 \pm 3.57$ $\mu \mathrm{g} / \mathrm{mL} 24$ hours after the clamp $(\mathrm{p}<0.001)$ and remained elevated till after a week $(p<0.001)$ compared to levels during euglycemia. This was similar for all subgroups. At all time-points, participants with type 2 diabetes had higher counts of granulocytes, lymphocytes and monocytes and higher hs-CRP compared to all other groups (all $\mathrm{p}<$ 0.01 ), whereas participants with type 1 diabetes had higher hs-CRP than controls $(\mathrm{p}=0.004)$.

\section{CONCLUSION}

A single hypoglycaemic event induces a prolonged immune response in humans independent of the presence of diabetes.

\section{5}

\section{Two years use of flash glucose monitoring is associated with sustained improvement of glycemic control and quality of life (FLARE-NL-6)}

Annel Lameijer ${ }^{1}$, Marion Fokkert ${ }^{2}$, Mireille Edens ${ }^{3}$, Reinold O.B. Gans ${ }^{4}$, Henk J.G. Bilo ${ }^{4,5}$, Peter R. van Dijk ${ }^{1}$

${ }^{1}$ University Medical Center Groningen, Department of Endocrinology, The Netherlands; ${ }^{2} I s a l a$, Department of Clinical Chemistry, Zwolle, The Netherlands; ${ }^{3}$ Isala, Department of Innovation and Science, Zwolle, The Netherlands; ${ }^{4}$ University Medical Center Groningen, Department of Internal Medicine, The Netherlands; ${ }^{5}$ Isala, Diabetes Research Center, Zwolle, The Netherlands

E-mail: a.lameijer@umcg.nl

\section{BACKGROUND}

The FreeStyle Libre (FSL) is a flash glucose monitoring (FGM) system. Previously the Flash Monitor Register in the Netherlands (FLARE-NL-4) study demonstrated positive effects of FSL-FGM use during one year on glycemic control, quality of life and disease burden among persons with diabetes mellitus (DM). The present follow-up study assesses the effects of FSL-FGM after two years.

\section{METHODS}

Patients included in the FLARE-NL4 study who continued FSL-FGM during the one-year study period were invited to participate $(n=687)$. Data were collected using questionnaires. 
(95\% CI -7.5, 2.7)): difference between groups 2.2 (95\% CI $-1.3,5.8) \mathrm{mmol} / \mathrm{mol}$. After two years, continued FSL-FGM

A total of 342 patients agreed to participate: mean age 48.0 ( \pm 15.6$)$ years, $52 \%$ men and $79.5 \%$ type $1 \mathrm{DM}$. HbA1c decreased from 60.7 (95\% CI 59.1, 62.3) $\mathrm{mmol} / \mathrm{mol}$ before the use of FSL-FGM to 57.3 (95\% CI $55.8,58.8) \mathrm{mmol} / \mathrm{mol}$ after one year and $57.8(95 \%$ CI 56.0, 59.5) after two years. At the end of the two-year follow-up period, 260 (76\%) persons still used the FSL-FGM and 82 (24\%) had stopped. Main reason for stopping FSL-FGM was financial constraints (55\%). Concerning the whole two-year period, there was a significant $\mathrm{HbAlc}$ decrease among persons who continued FSL-FGM (-3.5 mmol/mol (95\% CI -6.4, $-0.7)$ ), while HbA1c was unaltered compared to baseline among persons who stopped FSL-FGM $(-2.4 \mathrm{mmol} / \mathrm{mol}$ users had a higher SF-12 mental component score (MCS), a higher EQ-5D Dutch tariff score and felt less often anxious or depressed, compared to persons who discontinued FSL-FGM.

\title{
CONCLUSION
}

Although the considerable number of non-responders limit generalizability, this study suggests that persons who continue to use FSL-FGM for two years may experience sustained improvement of glycemic control and quality of life.

\section{6}

\section{Flash Glucose Monitoring in the Netherlands: increased monitoring frequency is associated with improvement of glycemic parameters}

\author{
Annel Lameijer ${ }^{1}$, Nicole Lommerde ${ }^{2}$, Timothy C. Dunn ${ }^{3}$, Marion J. Fokkert ${ }^{4}$, Mireille A. Edens ${ }^{5}$, Kalvin Kao ${ }^{3}$, Yongjin Xu ${ }^{3}$, R.O.B. Gans ${ }^{2}$, Henk J.G. Bilo ${ }^{2,6}$, \\ Peter R. van Dijk ${ }^{1,6}$ \\ ${ }^{1}$ University Medical Center Groningen, Department of Endocrinology, The Netherlands; ${ }^{2}$ University Medical Center Groningen, Department of Internal \\ Medicine, The Netherlands; ${ }^{3}$ Abbott Diabetes Care, Alameda, USA; ${ }^{4}$ Isala, Department of Clinical Chemistry, Zwolle, The Netherlands; ${ }^{5}$ Isala, Department \\ of Innovation and Science, Zwolle, The Netherlands; ${ }^{6}$ Isala, Diabetes Research Center, Zwolle, The Netherlands \\ E-mail:a.lameijer@umcg.nl
}

\section{BACKGROUND}

The FreeStyle Libre is a widely used system for flash glucose monitoring (FLASH). Insight into the use of FLASH under real life circumstances by larger groups is valuable to evaluate its effects. This study aims to evaluate the association between FLASH frequency and glycemic parameters during real-life circumstances in the Netherlands.

\section{METHODS}

Obtained glucose readings were de-identified and uploaded to a dedicated database when FLASH reading devices were connected to internet. Data between September 2014 and March 2020, comprising 16,331 analyzable readers $(163,762$ sensors) were analyzed. Scan rate per reader was determined and each reader was sorted into 20 equally sized rank ordered groups $(n=817$ each).

\section{RESULTS}

Users performed a median of 11.5 [IQR 7.7-16.7] scans per day. Those in the lowest and highest ventiles scanned on average 3.7 and 40.0 times per day and had an eHbAlc of $8.6 \%(71 \mathrm{mmol} / \mathrm{mol})$ and $6.9 \%(52 \mathrm{mmol} / \mathrm{mol})$, respectively. Increasing scan rates were associated with more time in target range (3.9-10 $\mathrm{mmol} / \mathrm{L})$, less time in hyperglycemia (> $10 \mathrm{mmol} / \mathrm{L}$ ), and a lower standard deviation of glucose. An eHbAlc of $7.0 \%$ (53 mmol/mol) translated in approximately $65 \%$ time in target range, $30 \%$ time in hyperglycemia and $5 \%$ time in hypoglycemia $(<3.9 \mathrm{mmol} / \mathrm{L})$.

\section{CONCLUSION}

These outcomes among Dutch FLASH users suggest that with higher scan rate glycemic control improves.

\section{7}

\section{Impact of the COVID-19 lockdown on behaviour, stress, anxiety and glycaemic control in patients with $\beta$-cell transplantation for type 1 diabetes}

\author{
C.P. Landstra, M.M. Ruissen, H. Regeer, M.F. Nijhoff, B.E.P.B. Ballieux, P.J.M. van der Boog, A.P.J. de Vries, S.D. Huisman, E.J.P. de Koning \\ Leiden University Medical Center, Leiden, The Netherlands \\ E-mail: c.p.landstra@lumc.nl
}


cCS bohn bohn
stafleu van loghum

SSS bohn

CL van loghum

SSS bohn

L van loghum

c) bohn stafleu

L van loghum

cer bohn stafleu

L van loghum

ceg bohn bohn
stafleu van loghum

c8h bohn stafleu van loghum

ç bohn stafleu van loghum

c) bohn stafleu van loghum

CS) bohn D) stafleu L. van loghum

cehn bohn stafleu van loghum
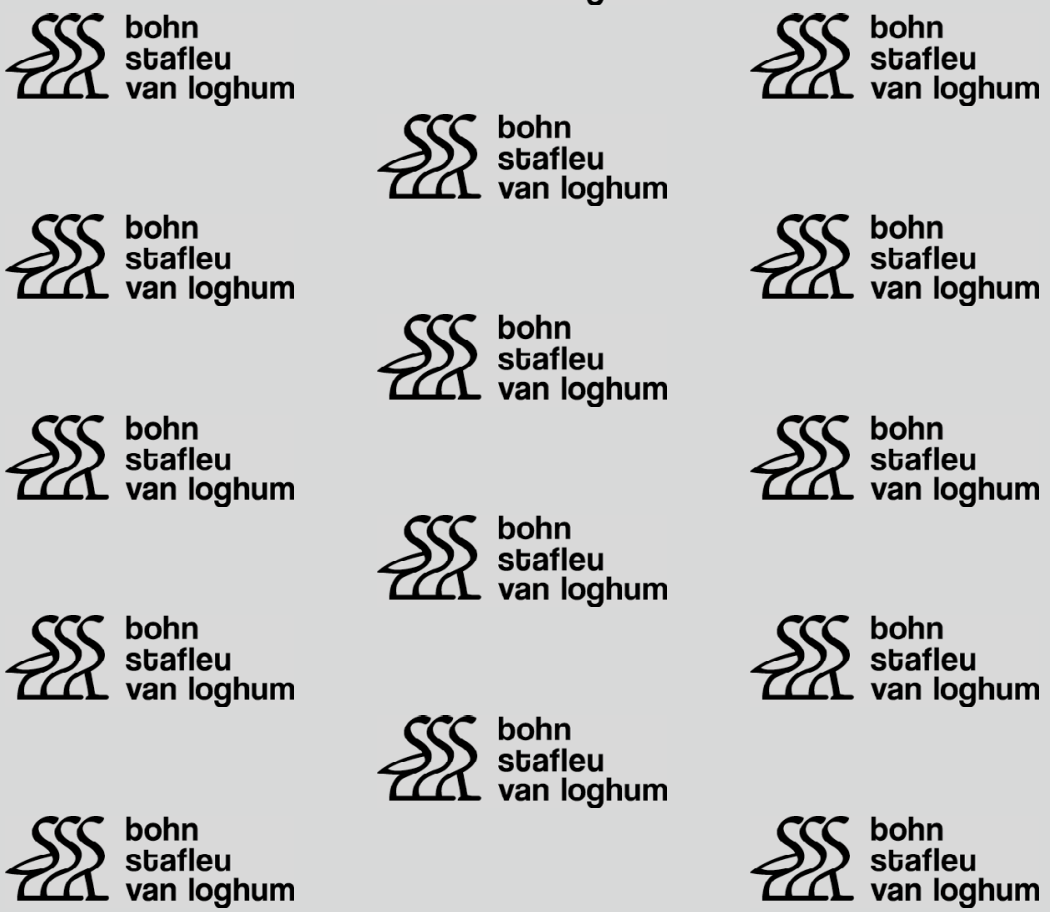

SS lohn

CI van loghum

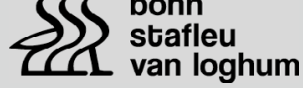

SSS bohn

SSS bohn

CSS bohn stafleu van loghum

SSS bohn stafleu van loghum

SSS bohn

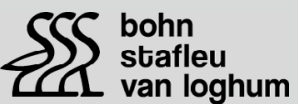

SSS bohn
SSS bohn stafleu van loghum
SSS lohn

SSS bohn

CL van loghum

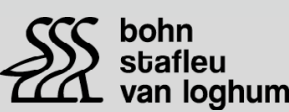

SSS bohn

CL van loghum

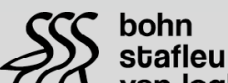

CL $\begin{aligned} & \text { stafleu } \\ & \text { van loghum }\end{aligned}$

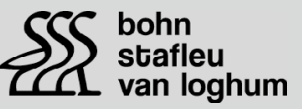

SSS bohn

IL van loghum

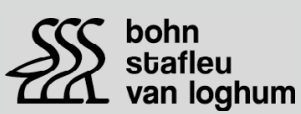

SSS bohn

CL van loghum

SSS lahn $\begin{aligned} & \text { bohn } \\ & \text { vanleu } \\ & \text { van loghum }\end{aligned}$

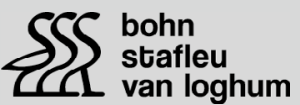

SSS bohn

CL van loghum bohn
stafleu Ll van loghum

SSS bohn II van loghum
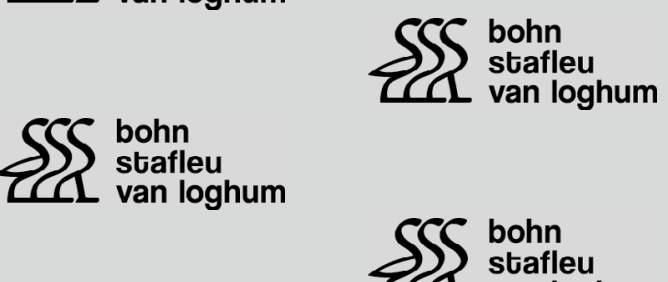
CL van loghum

CI van loghum

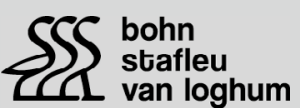

SSS bohn

Cl. van loghum
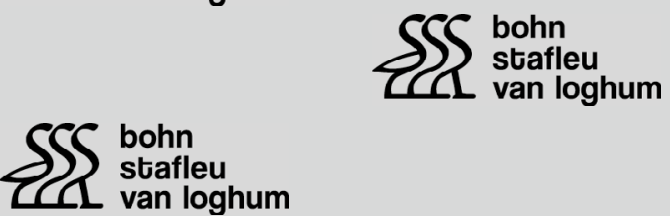

SSS bohn CL van loghum CL van loghum

SSS bohn

CL $\begin{aligned} & \text { stafleu } \\ & \text { van loghum }\end{aligned}$

SSL $\begin{aligned} & \text { bohn } \\ & \text { stafleu } \\ & \text { van loghum }\end{aligned}$

SSS bohn

CL van loghum

SSS bohn

CL van loghum

SSS bohn

CL van loghum

cSc bohn

$\sum$ stafleu

SSl bohn

van loghum

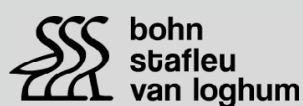

SCS bohn

$\sum \int$ stafleu

L van loghum

SSS bohn

CL van loghum

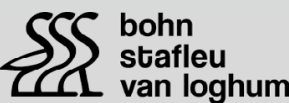

CL $\begin{aligned} & \text { stafleu } \\ & \text { van loghum }\end{aligned}$

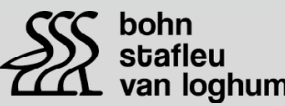

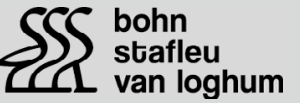
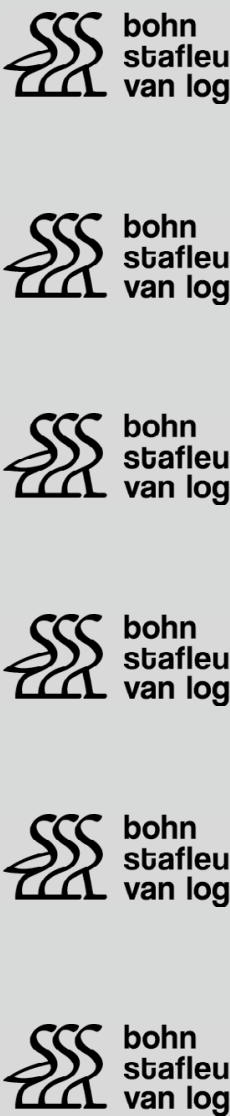

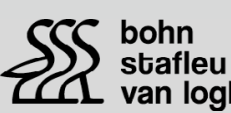

SSS $\begin{aligned} & \text { bohn } \\ & \text { stafleu } \\ & \text { van log }\end{aligned}$

SSS lohn

SSS bohn

ces bohn

CLL van log

L van log
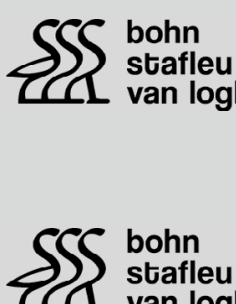

$3 \int \begin{aligned} & \text { bohn } \\ & \text { stafleu } \\ & \text { van log }\end{aligned}$

$\sum S \begin{aligned} & \text { bohn } \\ & \text { stafleu } \\ & \text { van log }\end{aligned}$ 
CS bohn

Stafleu

L van loghum

bohn

L van loghum

SSS bohn

II van loghum

bohn

5) stafleu

L van loghum

S bohn

stafleu

Lan loghum

Cohn

stafleu

L van loghum

SSS bohn

I van loghum

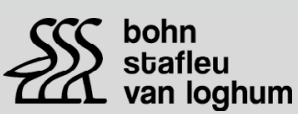

SSS bohn

CL van loghum

bohn

stafleu

Lan loghum
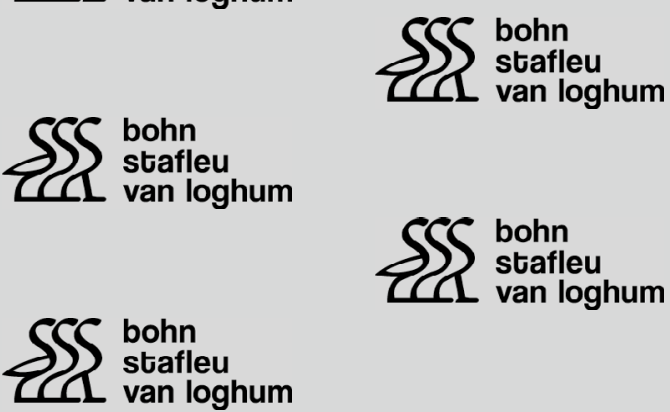

36 bohn

Stafleu

L van loghum

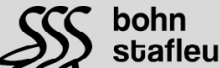

IL van loghum

3 bohn

stafleu

van loghum

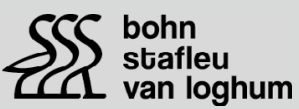

bohn

D) stafleu

Lan loghum

So bohn

stafleu

Lan loghum

SC bohn

stafleu

L van loghum

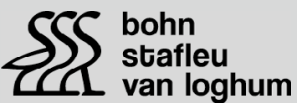

SSS bohn

Cl van loghum

SSS bohn

CL van loghum

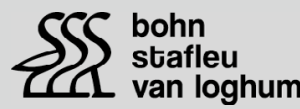

SSS $\begin{aligned} & \text { bohn } \\ & \text { stafleu }\end{aligned}$

CL van loghum

SSS bohn

CL van loghum

SCS bohn

bohn
stafleu

CL van loghum

SSS bohn

P) stafleu

IL van loghum

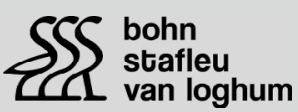

SSS bohn stafleu

van loghum

35 bohn

stafleu

L van loghum

SSS bohn

van loghum

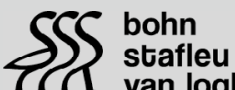

(1) stafleu

van loghum

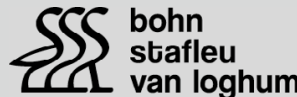

-) stafleu

3f bohn

stafleu

van loghum

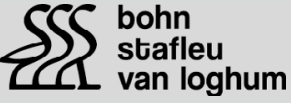

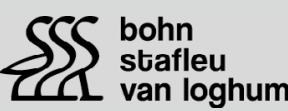
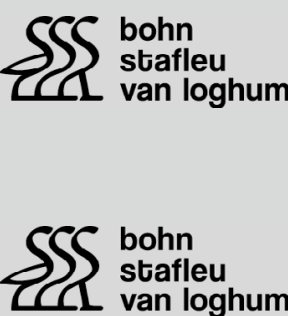

SSS bohn

CI van loghum

SSS bohn

¿L $\begin{aligned} & \text { stafleu } \\ & \text { van loghum }\end{aligned}$

SS lohn $\begin{aligned} & \text { bohn } \\ & \text { stafleu }\end{aligned}$

II van loghum

SSS bohn

Cl van loghum

ces bohn

IL van loghum

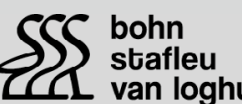

cCs bohn C) stafleu CLL van logh

SSS bohn CI van logh

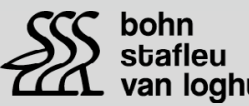

\&SS bohn stafleu van loghu

SSS bohn CI van loghu
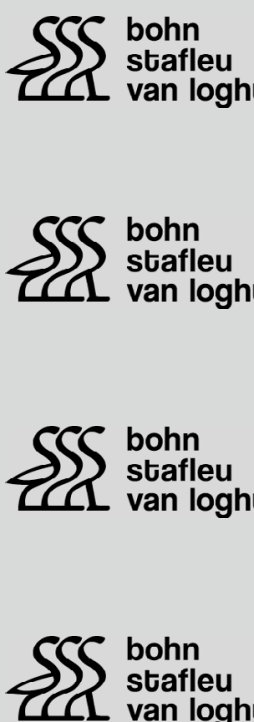

IL van logh
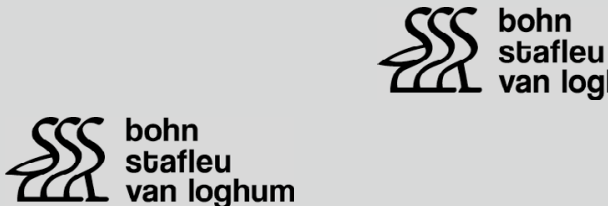

SSS lohn $\begin{aligned} & \text { bohn } \\ & \text { stafleu } \\ & \text { van loghum }\end{aligned}$

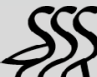

bohn stafleu

van loghu
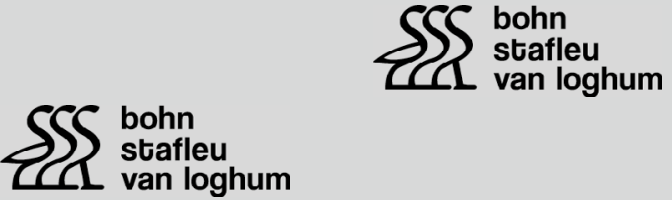


\section{BACKGROUND}

Patients with severely complicated type 1 diabetes (T1D) who receive $\beta$-cell transplantation have multiple risk factors for severe COVID-19 including the use of immunosuppression (IS). Lockdown strategies impact both mental and physical health, but this impact is expected even greater in patients at high risk for severe COVID-19. We therefore investigated the behavioural, mental and physical implications of the nationwide lockdown in islet and pancreas transplant $(\beta$-cell $\mathrm{Tx})$ recipients.

\section{METHODS}

To study the effect on glycaemic control, all patients with T1D and an islet transplantation or pancreas transplantation with non-optimal graft function using IS were eligible. As control group, patients with T1D without IS were included. Lockdown behaviour and self-reported changes in anxiety, stress, physical activity, weight, and glycaemic control were assessed using questionnaires. HbAlc and continuous glucose monitoring (CGM) metrics during lockdown were compared to before lockdown. isolation behaviour compared to patients with T1D alone $(\mathrm{n}=272)$, with $45.8 \%$ vs $14.0 \%(\mathrm{p}<0.001)$ reporting not leaving the house at all. A previous $\beta$-cell Tx was the most important predictor of isolating at home during the lockdown. Fear of coronavirus infection was higher in $\beta$-cell Tx recipients (VAS $5.0(3.0-7.0)$ vs 3.0(2.0-5.0), $\mathrm{p}=0.004)$ and glycaemic control worsened during lockdown as assessed by HbAlc $(\Delta \mathrm{HbA1c}+1.67 \pm 8.74$ vs $-1.72 \pm 6.15 \mathrm{mmol} /$ mol, $\mathrm{p}=0.006)$ as well as CGM $(\Delta$ time in range $-4.5 \%$ $(-6.0 \%-1.5 \%)$ vs $+3.0 \%(-2.0 \%-6.0 \%), \mathrm{p}=0.038)$. Among $\beta$-cell Tx recipients, $29.2 \%$ reported more difficulty with glycaemic control, $40.0 \%$ decreased physical activity, $41.7 \%$ weight gain, $29.2 \%$ increased anxiety and $33.3 \%$ increased stress since the lockdown started.

\section{CONCLUSION}

The COVID-19 pandemic and subsequent lockdown add additional fear of infection, deterioration of glycaemic control and more stringent social isolation behaviour when patients with T1D also have a $\beta$-cell Tx. Health care professionals should be aware of these behavioural, mental and physical implications to provide extra support.

\title{
RESULTS
}

$\beta$-cell Tx recipients $(n=51)$ showed more stringent social

8

\section{Sustained improvement of glycaemic outcomes in people with type 1 diabetes one year into the COVID-19 pandemic}

\author{
Namam Ali ${ }^{1}$, Soumia El Hamdaoui ${ }^{1}$, Giesje Nefs ${ }^{2,3,4}$, Cees J. Tack ${ }^{1}$, Bastiaan E. de Galan ${ }^{1,5,6}$ \\ ${ }^{1}$ Department of Internal Medicine, Radboud University Medical Center, Nijmegen, The Netherlands; ${ }^{2}$ Radboud Institute for Health Sciences, Department of \\ Medical Psychology, Radboud University Medical Center, Nijmegen, The Netherlands; ${ }^{3}$ Center of Research on Psychological disorders and Somatic diseases \\ (CoRPS), Department of Medical and Clinical Psychology, Tilburg University, Tilburg, The Netherlands; ${ }^{4}$ Diabeter, National treatment and research center for \\ children, adolescents and adults with type 1 diabetes, Rotterdam, The Netherlands; ${ }^{5}$ Department of Internal Medicine, Maastricht University Medical \\ Center+, Maastricht, Tthe Netherlands; ${ }^{6}$ CARIM School for Cardiovascular Disease, Maastricht University, Maastricht, The Netherlands \\ E-mail: Namam.Ali@radboudumc.nl
}

\section{BACKGROUND}

Since the start of the COVID-19 pandemic, several lockdown measures have been implemented, including changes in health care accessibility, that could have affected health outcomes for people with type 1 diabetes. A few studies examined short-term glycaemic effects of the first COVID-19 wave, but no study addressed effects of following COVID-19 waves. This study investigated, in people with type 1 diabetes, parameters of glycaemic control one year after the first wave of the COVID-19 pandemic and determined factors associated with improvement.

\section{METHODS}

This was an observational cohort study in people with type 1 diabetes, aged $\geq 16$ years. We compared $\mathrm{HbAlc}$ and flash glucose monitoring (FGM) glucometrics between the prelock-down period and after one-year of follow-up. Using regression analysis, we assessed baseline factors associated with $\mathrm{HbA} 1 \mathrm{c}$ reduction of at least $5 \mathrm{mmol} / \mathrm{mol}$.

\section{RESULTS}

A total of 330 people with type 1 diabetes participated in the study. After one year of follow-up after the COVID-19 lockdowns, mean $( \pm \mathrm{SD})$ HbA1c decreased from $63.5 \pm$ 11.0 to $58.8 \pm 9.8 \mathrm{mmol} / \mathrm{mol}(\mathrm{p}<0.001)$. The time in target range (glucose, $3.9-10.0 \mathrm{mmol} / \mathrm{l}$ ) increased from $55.8 \pm$ 17.7 to $58.4 \pm 17.2 \%(\mathrm{p}=0.013)$. The proportion of times with glucose $\leq 3.9 \mathrm{mmol} / \mathrm{l}$ or $>13.9 \mathrm{mmol} / 1$ fell from $5.0 \pm$ 5.0 to $3.7 \pm 3.8 \%$ and from $15.0 \pm 14.1$ to $12.6 \pm 11.8 \%$, respectively (both $\mathrm{p}<0.05$ ). Glucose variability fell from 38.5 \pm 7.5 to $36.5 \pm 6.0 \%(\mathrm{p}<0.001)$ and the median (IQR) 
duration of hypoglycaemic events decreased from 90 [69$118]$ to 76 [59-91] minutes ( $\mathrm{p}<0.001)$. The proportion of time the sensor is active increased from $77.6 \pm 29.7$ to 87.2 $\pm 22.6 \%(\mathrm{p}<0.001)$. In regression analysis, smoking, FGM use and higher $\mathrm{HbAlc}$ at baseline were associated with an $\mathrm{HbAlc}$ decrease of at least $5 \mathrm{mmol} / \mathrm{mol}$.

\section{CONCLUSION}

This study shows improvement in a range of glycaemic outcomes in individuals with type 1 diabetes over one year after the start of the COVID-19 pandemic, particularly in smokers and participants using FGM and with higher HbAlc.

\title{
9
}

\section{A common variant in the ketohexokinase gene is associated with fructosuria and cardiometabolic outcomes}

\begin{abstract}
A.M Buziau, C.G. Schalkwijk, J.L.J.M. Scheijen, P.I.H.G. Simons, C.J.H. van der Kallen, S.J.P.M. Eussen, N.C. Schaper, P.C. Dagnelie, M.M.J. van Greevenbroek, A. Wesselius, C.D.A. Stehouwer, M.C.G.J. Brouwers
\end{abstract}

Maastricht University, Maastricht University Medical Centre+, CARIM, Maastricht, The Netherlands

\section{BACKGROUND}

There is an ongoing discussion on whether fructose per se is disadvantageous for cardiometabolic health. Recently, pharmacological inhibition of ketohexokinase (KHK), the first enzymatic step of fructolysis, was shown to reduce intrahepatic lipid content in humans. The study of individuals carrying functional variants in the KHK gene will provide more insight into the lifelong effects of inhibition of $\mathrm{KHK}$, as these individuals have been exposed from birth to a KHK protein with less enzymatic activity. The aims of the present study were: 1) to study whether a common missense variant in KHK (rs2304681) is a functional variant that affects fructosuria (similar to pharmacological inhibition of KHK); and 2) to study the association between rs2304681 and cardiometabolic outcomes.

\section{METHODS}

First, linear regression analyses were performed to study the association between rs 2304681 and 24 h urinary fructose levels (quantified by tandem mass spectrometry), with adjustment for age, sex, and type 2 diabetes (T2D), in a population-based cohort (the Maastricht Study, $\mathrm{n}=1,471$ ). Second, we used summary-level data on the association of rs2304681 with T2D, hypertension, and myocardial infarction, obtained from publicly available databases.

\section{RESULTS}

First, the rs2304681 minor A allele (frequency: 0.36) was associated with higher $24 \mathrm{~h}$ urinary fructose levels (unstandardized beta: 0.064; 95\%CI: 0.027-0.100). There was no statistical interaction between rs2304681 and T2D (P for interaction $=0.61)$. Second, fixed effects meta-analyses showed protective effects of the rs2304681 minor A allele on T2D (OR: $0.985 ; 95 \%$ CI: 0.975-0.99; $\mathrm{n}=1,331,670$ ) and myocardial infarction (OR: 0.976; 95\% CI: 0.961-0.992; $\mathrm{n}=$ $583,191)$. Two studies both showed a protective effect on the risk of hypertension (OR: 0.988; 95\% CI: 0.976-0.999; n $=440,285$ and Z-score: $-2.59 ; \mathrm{n}=192,763)$.

\section{CONCLUSION}

Lifelong impairment of KHK activity (reflected by rs2304681) is associated with fructosuria and protection from cardiometabolic disease. These findings suggest that fructose per se has harmful cardiometabolic effects, which may be mitigated by pharmacological inhibition of KHK.

10

\section{Older age, polypharmacy, and low systolic blood pressure are associated with more hypotension- related adverse events in patients with type 2 diabetes treated with antihypertensives}

\author{
Martina Ambrož ${ }^{1}$, Sieta de Vries ${ }^{1}$, Klaas Hoogenberg ${ }^{2}$, Petra Denig ${ }^{1}$ \\ ${ }^{1}$ Department of Clinical Pharmacy and Pharmacology, University Medical Center Groningen, Groningen, The Netherlands; ${ }^{2}$ Department of Internal \\ Medicine, Martini Hospital, Groningen, The Netherlands
}

\section{BACKGROUND}

Low systolic blood pressure (SBP) levels while being treated with antihypertensives may cause hypotension-related adverse events (hrAEs), especially in the elderly, women, and frail patients. We wanted to assess the association between the occurrence of hrAEs and low SBP levels, age, sex, and polypharmacy among type 2 diabetes (T2D) patients treated with antihypertensives. 


\section{METHODS}

We used the Groningen Initiative to ANalyse Type 2 diabetes Treatment (GIANTT) database which includes primary care T2D patients from the north of the Netherlands. Patients treated with $\geq 1$ antihypertensive drug and $\geq 1$ SBP measurement between 2012 and 2014 were included. The outcome was the presence of an hrAE (postural hypotension, dizziness, weakness/tiredness, and syncope) in 90 days before or after the lowest recorded SBP level. Age $(\geq$ 70 vs. $<70$ years), sex (women vs. men), polypharmacy ( 5 to 9 drugs or $\geq 10$ drugs vs. $<5$ drugs), and SBP level $(<130$ or $\geq 130 \mathrm{mmHg}$ ) were included as determinants. Logistic regression analyses were conducted for age, sex and polypharmacy, including the SBP level and their interaction, adjusted for confounders. level $<130 \mathrm{mmHg}$ and $5.4 \%$ experienced an hrAE. Patients with an SBP level $<130 \mathrm{mmHg}$ had a significantly higher occurrence of hrAEs than patients with a higher SBP level (6.2\% vs. $4.0 \%$; $\mathrm{p}<0.014)$. Older patients $(6.6 \%$ vs. $4.2 \%$; $\mathrm{p}$ $<0.032)$ and patients with polypharmacy $(<5$ drugs $3.8 \%$ vs. 5 - 9 drugs $5.8 \%$ vs. $\geq 10$ drugs $10.0 \%$; p < 0.050 ) were more likely to experience an hrAE. The association with sex and the interactions between the determinants and SBP level were not significant.

\section{CONCLUSION}

Older patients and those with polypharmacy are particularly at risk of hrAEs. Possible negative effects of treatment to low SBP targets should be regularly evaluated in all T2D patients.

\title{
RESULTS
}

We included 21,119 patients, $49 \%$ were $\geq 70$ years old, $52 \%$ were women, $57 \%$ had polypharmacy, $61 \%$ had an SBP

\section{1}

\section{Sex disparities in the quality of medication treatment among patients with type 2 diabetes}

\author{
Marit Geelink ${ }^{1}$, Martina Ambrož ${ }^{1}$; Kirsten Smits² , Sieta de Vries ${ }^{1}$, Petra Denig ${ }^{1}$
}

${ }^{1}$ Department of Clinical Pharmacy and Pharmacology, University Medical Center Groningen, Groningen, The Netherlands; ${ }^{2}$ Department of Dentistry, Radboud University Medical Center, Nijmegen, The Netherlands

BACKGROUND

Sex differences in risk factor levels and clinical outcomes have been observed for patients with type 2 diabetes (T2D). Whether these differences might be due to disparities in medication prescribing is not clear. We wanted to determine whether there have been sex disparities in medication prescribing among patients with T2D.

\section{METHODS}

A cohort study was conducted using the Groningen Initiative to ANalyze Type 2 diabetes Treatment (GIANTT) database, which includes data of primary care patients with T2D from the north of the Netherlands. Data on demographics, physical examinations, laboratory measurements and prescribing were extracted for 2012 and 2014. A set of validated prescribing quality indicators (PQIs) assessing prevalent medication, starting, and intensifying medication, and safety of glucose-, lipid-, blood pressure- and albuminuria-lowering medication were used to assess potential sex differences by multivariate logistic regression analyses, adjusted for age and body mass index.

\section{RESULTS}

We included 22,853 patients ( $50.5 \%$ women). Women were less often treated with metformin $(85.5 \%$ vs. $88.3 \%$; OR 0.77, 95\% CI 0.70-0.85), and were less often prescribed a renin angiotensin aldosterone inhibitor (RAAS-i) when treated with multiple blood pressure lowering medicines (85.3\% vs. $90.1 \%$; OR $0.66,95 \%$ CI $0.59-0.73$ ) or when having albuminuria $(81.3 \%$ vs $85.8 \%$; OR $0.78,95 \%$ CI 0.63 $0.96)$. Statin treatment was less frequently started $(30.6 \%$ vs. $35.2 \%$; OR $0.82,95 \%$ CI $0.70-0.95)$ and intensified (44.1\% vs. $48.5 \%$; OR $0.84,95 \%$ CI $0.71-1.00$ ) in women. There were no sex differences in starting or intensifying glucose-, blood pressure- and albuminuria-lowering medication or in safety-related issues.

\section{CONCLUSION}

Sex disparities in medication prescribing among T2D patients were seen, including potential undertreatment with RAAS-i and starting and intensifying statin treatment in women. Such disparities may in part explain the higher risks for cardiovascular and renal complications observed in women. Further research is needed to assess whether sex disparities have changed over time. 


\title{
Seven weeks of oral histidine supplementation improves glycemic control in type 2 diabetes patients
}

\author{
M.V. Warmbrunn' ${ }^{1 *}$, I. Attaye ${ }^{1}$, J. Aron-Wisnewsky ${ }^{1}$, M. Aerts ${ }^{1}$, M.R. Soeters ${ }^{2}$, D.R. van Raalte ${ }^{3}$, A.K. Groen ${ }^{1}$, F. Backhed ${ }^{4}$, M. Nieuwdorp ${ }^{1,4}$ \\ ${ }^{1}$ Department of Internal and Vascular Medicine, Amsterdam University Medical Centers, Location AMC, Amsterdam, The Netherlands; ${ }^{2}$ Department of \\ Endocrinology and Metabolism, Amsterdam UMC, Location AMC at University of Amsterdam, Amsterdam, The Netherlands; ${ }^{3}$ Diabetes Center, \\ Department of Internal Medicine, Amsterdam UMC, Location VUMC at VU University Medical Centers, Amsterdam, The Netherlands; ${ }^{4}$ Wallenberg \\ Laboratory, University of Gothenburg, Göteborg, Sweden \\ ${ }^{*}$ Corresponding author: M.V. Warmbrunn \\ E-mail:m.v.warmbrunn@amsterdamumc.nl
}

\section{BACKGROUND}

In-vivo and in-vitro studies have suggested that histidine could drive metabolic alterations in obese type $2 \mathrm{DM}$ (T2D) humans via the production of imidazole proprionate $(\operatorname{ImP})$, the production of which might be driven via an altered gut microbiota composition. Since there are currently no data on direct oral histidine supplementation on several markers of cardiometabolism in obese humans with T2D nor on its related metabolite production of ImP by the gut microbiota. We aim to study pharmacokinetic levels of plasma ImP levels as a result of orally administrated histidine and assess the role of the gut microbiome in this respect.

\section{METHODS}

Interventional controlled study with 44 subjects: 22 healthy controls (11 Caucasian and 11 of SAS ethnicity), 22 type 2 diabetes mellitus (11 Caucasian and 11 of SAS ethnicity) on stable metformin use. Subjects take $4 \mathrm{~g}$ of oral histidine once a day for seven weeks. After the first two weeks, seven days of antibiotics (vancomycine, ciprofloxacine \& metronidazole) will be added to suppress the gut microbiome followed by a four weeks recovery period. Plasma and urinary $\operatorname{ImP}$, histidine, urocanate and glutamate levels will be measured as well as glucose and insulin levels.

\section{RESULTS}

An interim analysis of $\mathrm{n}=10$ subjects with $\mathrm{T} 2 \mathrm{D}$ revealed an increase of serum histidine by $1.76 \mu \mathrm{mol} / \mathrm{L}$ [95\% CI 0.75 to 2.78 ] after two weeks. After 7 weeks, there was a significant decrease of fasting plasma glucose $(-1.80 \mathrm{mmol} / \mathrm{L}$ [95\% CI -2.91 to -0.69$])$ and HOMA-IR (-2.61 [95\% CI -4.40 to -0.82$])$. After microbiota depletion, there was also an increase in plasma $\mathrm{ImP}(1239.57 \mathrm{nmol} / \mathrm{L}$ [95\% CI 770.07 to 1709.08]), compared to baseline. In addition to this, systolic blood pressure $(-11.85 \mathrm{mmHg}$ [95\%CI -18.76 to $-4.93])$ and diastolic blood pressure $(-5.58 \mathrm{mmHg}$ [95\% CI -9.49 to -1.66$]$ ) was decreased.

\section{CONCLUSION}

Oral histidine intake increases plasma histidine but not ImP levels, however, broadspectrum antibiotic treatment increases ImP levels. Furthermore, histidine intake improves glycemic control over time.

13

\section{Diabetes complications are associated with an altered inflammatory state in type 1 diabetes: a proteomics approach}

\author{
Mandala Ajie ${ }^{1}$, Julia van Heck, Rinke Stienstra, Cees Tack ${ }^{1}$ \\ ${ }^{1}$ Department of Internal Medicine, Radboudumc, Nijmegen, The Netherlands
}

\section{BACKGROUND}

Type 1 diabetes can lead to the development of vascular complications. Vascular complications are known to be partly driven by an altered inflammatory state. In this study, we aimed to identify a link between disease characteristics and inflammatory state characterized by a proteomic approach in patients with type 1 diabetes.

METHODS

243 type 1 diabetes patients (M:F ratio 1.17 , age $52 \pm 16$ years, duration of diabetes $28 \pm 16$ years, BMI $25.8 \pm 4.4$, mean HbAlc $66 \pm 13 \mathrm{mmol} / \mathrm{mol}$ ) were recruited in this cross-sectional study. Blood samples were drawn and the levels of circulating inflammatory proteins in the serum were measured using 96-plex inflammatory panel proximity extension assay (Olink Proteomics, Uppsala, Sweden. Statistical analyses were performed using R programming language (version 4.0.3).

\section{RESULTS}

Diabetes duration was positively correlated with circulating inflammatory proteins in our cohort. 27/92 proteins 
were positively correlated with duration of diabetes. Pathway enrichment analysis showed strong correlations between the proteins and immune cell migration ( $\mathrm{p}$-value $\left.1.75 \times 10^{-11}\right)$ and a number of signaling pathways such as ERK1 and ERK2 cascade (p-value $3.89 \times 10^{-10}$ ). Glycemic control (HbA1c) positively affected the levels of $10 / 92$ proteins in the panel. No association was found between circulating inflammatory proteins and other characteristics.

We also found strong correlations between diabetic complications and elevated inflammatory markers, independent of diabetes duration and glycemic control. Nephropathy was positively associated with $16 / 94$ proteins. Nephropathy was shown to be particularly enriched in proteins related to positive chemotaxis ( $\mathrm{p}$-value $3.60 \times 10^{-4}$ ) and Ras signaling (p-value 0.019). Stroke was associated with $11 / 92$ proteins, of which 9 proteins overlapped with the ones associated with nephropathy.

\section{CONCLUSION}

Glucose control (HbAlc) and diabetes duration seem to drive an enhanced inflammatory state in T1D patients. Moreover, diabetic complications are independently associated with increased circulating levels of several inflammatory proteins. Future studies are needed to determine the mechanisms of action underlying the association between the identified pathways, such as Ras cascade and chemokine signaling and diabetic complications.

\title{
14
}

\section{Increased fat and inflammation in livers of deceased DM patients without diagnosed liver disease}

\author{
A. Korn ${ }^{1,2}$, E. Bos ${ }^{1}$, C. Nadeem ${ }^{1}$, H.W.M. Niessen ${ }^{1,2,3}$, S. Simsek ${ }^{4,5}$, P.A.J. Krijnen ${ }^{1,2}$ \\ ${ }^{1}$ Department of Pathology, Amsterdam University Medical Centre (AUMC) Location VUmc, Amsterdam, The Netherlands; ${ }^{2}$ Amsterdam Cardiovascular Sciences, \\ Amsterdam, The Netherlands; ${ }^{3}$ Department of Cardiac Surgery, Amsterdam UMC Location VUmc, Amsterdam, The Netherlands; ${ }^{4}$ Department of Internal \\ Medicine, Northwest Clinics, Alkmaar, The Netherlands; ${ }^{4}$ Department of Internal Medicine, Amsterdam UMC Location VUmc, Amsterdam, The Netherlands \\ E-mail:a.korn@amsterdamumc.nl
}

\section{BACKGROUND}

Diabetes has been strongly associated with non-alcoholic fatty liver disease (NAFLD), which increases risk of severe liver disease as well as extra-hepatic microvascular disorders. NAFLD includes excessive fat accumulation and increased inflammation, but is often diagnosed relatively late due to limitations of current diagnostic techniques and light clinical manifestations in the early stages of development. This study aimed to analyse hepatic fat and inflammation in DM patients without previous diagnosis of NAFLD.

\section{METHODS}

Liver tissue was obtained at autopsy from $34 \mathrm{DM}$ patients and 69 non-diabetic control patients. The hepatic fat (percentage and amount of cells) and inflammatory cell markers CD45 and CD68 were studied through immunohistochemical analysis.

\section{RESULTS}

We observed a 5-fold increase in fat percentage (3.8 \pm 7.4 , $\mathrm{p}<0.0001)$ and an 8 -fold increase in amount of fat cells in the livers of DM patients $(233.3 \pm 342.3$, p $<0.0001)$ compared to the non-diabetic controls (fat percentage: $0.8 \pm$ 2.6; fat cells: $29.7 \pm 77.5)$. This increase was consistently observed when separating patients on sex or age. CD45 was decreased in the diabetic livers $(p=0.003)$, whereas CD68 was increased in DM patients $(\mathrm{p}=0.001)$ compared to the controls. When separating patients on sex, similar results were observed, whereas age separation showed no differences between diabetic or non-diabetic liver tissue. No correlation was found between hepatic fat and inflammation.

\section{CONCLUSION}

DM patients showed significantly more hepatic fat, both in percentage and in amount of cells, regardless of sex and age. Additionally, presence of macrophages was increased. These data indicate that hepatic fat and inflammation are also increased in DM patients that are not diagnosed with NAFLD, and suggest early intervention could be helpful to reduce further development of hepatic or extra-hepatic disease. 


\title{
The association between muscle mass and diabetes in different weight groups
}

\author{
D. Sizoo ${ }^{1,2}$, L. de Heide' ${ }^{1}$, M. Emous' ${ }^{1}$, T. van Zutphen ${ }^{2}$, E.N van Roon ${ }^{1}$, A. van Beek ${ }^{3}$ \\ ${ }^{1}$ Medical Center Leeuwarden, Leeuwarden, The Netherlands; ${ }^{2}$ Campus Fryslân, University of Groningen, Leeuwarden The Netherlands; ${ }^{3}$ Department of \\ Endocrinology, Universitair Medisch Centrum Groningen, Groningen, The Netherlands \\ E-mail: D.sizoo@rug.nl
}

\section{BACKGROUND}

In the elderly, low muscle mass has gained considerable attention as a predictor for type 2 diabetes and mortality. In obesity, lower muscle mass is also associated with increased mortality. However, it is unknown how muscle mass relates to several comorbidities throughout a wide range of weight classes. We therefore aimed to examine the association between muscle mass and obesity-related comorbidities in different weight classes.

\section{METHODS}

Adult participants were included from the Lifelines cohort study. Muscle mass was estimated with 24-hour creatinine excretion and stratified for gender. Low muscle mass was defined as $<-1$ SD of the creatinine excretion, and compared to high muscle mass ( $>1 \mathrm{SD}$ ). Multivariate logistic regression analysis was used to assess the association between muscle mass and diabetes, stratified by gender and BMI groups and corrected for age, waist- and hip circumference.

\section{RESULTS}

The population consisted of 132,714 participants $(55,385$ male and 77,329 female), with a respective mean age of 44.9 and 43.9. In males, $13.9 \%$ had a low muscle mass and $14.1 \%$ of the females had a low muscle mass. In table 1 , the
Table 1. Multivariate logistic regression of the association between muscle mass and diabetes, correct for age, waist- and hip circumference.

\begin{tabular}{lcccc} 
& OR & Cl lower & Cl higher & p-value \\
\hline Multivariate & 1.76 & 1.49 & 2.08 & $<0.001$ \\
\hline Male & 2.12 & 1.62 & 2.78 & $<0.001$ \\
BMI 18.5-25 & 3.08 & 1.19 & 7.95 & 0.02 \\
BMI 25-30 & 1.78 & 1.23 & 2.57 & 0.002 \\
BMI 30+ & 1.64 & 1.11 & 2.43 & 0.014 \\
\hline Female & 1.49 & 1.2 & 1.86 & $<0.001$ \\
BMI 18.5-25 & 1.22 & 0.7 & 2.11 & 0.491 \\
BMI 25-30 & 1.3 & 0.9 & 1.88 & 0.165 \\
BMI 30+ & 1.48 & 1.10 & 2.0 & 0.009 \\
\hline
\end{tabular}

regression results are shown. Overall, males had an higher OR for diabetes (in association with low muscle mass) than females. In males, the OR for diabetes increased with decreasing BMI class whereas in females the effect was highest in the group BMI $30+$.

\section{CONCLUSION}

Our findings show that diabetes is associated with a lower muscle mass, especially in males. This effect varies between the different BMI groups and genders, with higher risks in lower BMI classes for males.

\section{Supplementation of methylglyoxal in drinking water does not affect the cerebral microvasculature and cognitive function in non-diabetic mice}

\author{
Eline Berends ${ }^{1,2}$, Naima Amiri, ${ }^{1,2}$, Marjo P.H. van de Waarenburg ${ }^{1,2}$, Jean L.J.M. Scheijen ${ }^{1,2}$, Denise J.H.P. Hermes ${ }^{3,6}$, Robert J. van 0ostenbrugge ${ }^{2,4}$, \\ Sébastien Foulquier ${ }^{2,5,6 *}$, Casper G. Schalkwijk ${ }^{1,2 *}$ \\ ${ }^{1}$ Department of Internal Medicine, Maastricht University, Maastricht, The Netherlands; ${ }^{2}$ School for Cardiovascular Diseases (CARIM), Maastricht, The \\ Netherlands; ${ }^{3}$ Department of Psychiatry and Neuropsychology, Maastricht University, Maastricht, The Netherlands; ${ }^{4}$ Department of Neurology, Maastricht \\ University Medical Centre, Maastricht, The Netherlands; ${ }^{5}$ Department of Pharmacology and Toxicology, Maastricht University, Amsterdam, The \\ Netherlands; ${ }^{6}$ School for Mental Health and Neuroscience, Maastricht, The Netherlands \\ *Shared last-authorship \\ E-mail: e.berends@maastrichtuniversity.nl
}

\section{BACKGROUND}

Diabetes is associated with cerebral small vessel disease (cSVD) and cognitive impairment, however, the underlying mechanism remains unclear. Methylglyoxal (MGO), a by-product of glycolysis and a precursor in the formation of advanced glycation end products (AGEs), is increased in diabetes and associated with microvascular dysfunction. Our group has previously shown that MGO is increased in brain tissue of diabetic rats. We therefore investigated the role of increased levels of circulating MGO microvascular inflammation and 
cognitive function.

\section{METHODS}

2-3 months old male $\mathrm{C} 57 \mathrm{Bl} / 6 \mathrm{~J}$ mice were treated with MGO (50 mmol/l, drinking water) or not (control) for 3 months ( $\mathrm{n}=17$ per group), during which a range of cognitive tests were performed. After sacrifice, MGO and AGEs in plasma and brain were measured by UHPLC-MS/MS, plasma inflammatory markers were measured by ELISA and cortical microvessels were isolated for further analysis.

\section{RESULTS}

In the MGO group, plasma MGO was increased 2-fold ( $\mathrm{p}<$ 0.0001 ) and free plasma MGO-derived hydroimidazolone-1 (MG-H1) and Ne-(1-carboxyethyl)lysine (CEL) were increased 1.2 -fold $(\mathrm{p}=0.01)$ and 1.7 -fold $(\mathrm{p}=0.01)$, respectively. In brain tissue, there was a 1.4-fold and 1.1- fold increase in free MG-H1 $(p=0.02)$ and CEL $(p=0.001)$ in comparison to controls. Cognitive function remained unchanged after MGO treatment. MGO did not change expression of plasma inflammatory markers IFN- $\gamma$, IL-10, IL-1 $\beta$, IL- 6 , TNF- $\alpha$ and CXCL1. In cortical microvessels, expression of inflammatory genes VCAM-1, ICAM-1, sirtuin-1 and AGE receptor, were unchanged.

Plasma MGO and MGO-derived AGEs and brain MGO-derived AGEs were increased by supplementation of MGO in drinking water to a level comparable to that in diabetes. This was however not associated with microvascular inflammation and cognitive impairment.

\section{CONCLUSION}

Our findings indicate that increased levels of circulating MGO by itself does not cause microvascular inflammation nor cognitive decline.

\title{
17
}

\section{An integrated e-health support of shared decision-making and self-management (POWER2DM) for patients with diabetes mellitus and their healthcare professionals}

\author{
Merel Ruissen ${ }^{1}$, José David Torres-Peña, Bas Uitbeijerse, Antonio Pablo Arenas de Larriva, Sasja Huisman, Albert de Graaf, Eelco de Koning, \\ Javier Delgado-Lista, Jacob Sont ${ }^{1}$, POWER2DM Consortium \\ ${ }^{1}$ Leiden University Medical Center, Leiden, The Netherlands \\ E-mail:m.m.ruissen@lumc.nl
}

\section{BACKGROUND}

Although there are several e-health systems that aim to support diabetes care, systems that integrate the complex variety of aspects relevant for diabetes self-management (DSM) are still lacking. We developed and field-tested an e-health system integrating medical, psychological and behavioral aspects and connected wearables to support patients and healthcare professionals in shared decision-making and DSM (POWER2DM).

\section{METHODS}

Patients with type 1 (T1DM) and type 2 diabetes (T2DM) were recruited from hospital outpatient diabetes clinics in The Netherlands and Spain and randomized to POWER2DM or usual care for 37 weeks. We assessed the change in HbAlc, quality of life (QoL) (WHO-5) and DSM (DSMQ-R) and compared outcomes between the POWER2DM and usual care group.

\section{RESULTS}

In total 226 patients were included (T1DM: $\mathrm{n}=108$, T2DM: $\mathrm{n}=118$ ). In the POWER2DM group HbA1c decreased from
$60.6(\mathrm{SD} 14.7) \mathrm{mmol} / \mathrm{mol}(7.7(1.3) \%)$ at baseline to 56.7 (12.1) $\mathrm{mmol} / \mathrm{mol}(7.3(1.1) \%)(\mathrm{p}<0.001)$, with no significant change in $\mathrm{HbAlc}$ in the usual care group (between-group difference: $-0.24 \%, p=0.008$ ). Subgroup analysis showed an improvement in $\mathrm{HbAlc}$ in patients with T2DM $(-0.63 \%, \mathrm{p}<0.001)$, but not in patients with T1DM $(0.01 \%, \mathrm{p}=0.84)$. QoL improved in patients with T1DM in the POWER2DM group compared to usual care (baseline: 15.7 (3.8), end: 16.3 (3.5), between-group difference: $\mathrm{p}=$ 0.047), but not in patients with T2DM. DSM improved in both patients with T1DM (7.3 (1.2) to 7.7 (1.2), p < 0.005) and T2DM (6.5 (1.3) to 6.7 (1.3), p < 0.01) within the POWER2DM group, without a significant difference between the POWER2DM and usual care group.

\section{CONCLUSION}

POWER2DM improves HbAlc compared to usual care in patients with T2DM and improves QoL in patients with T1DM. DSM tended to improve in all patients with diabetes, without a significant between-group difference. 


\title{
Opinions about blood pressure care and therapy adherence on the level of healthcare providers and healthcare system: a qualitative study
}

\author{
S.E. Van Grondelle' ${ }^{1}$ S. van Bruggen ${ }^{1}$, J. Meijer ${ }^{1}$, A.T.A. Mairuhu' ${ }^{5}$, Y.W.J. Sijpkens ${ }^{4}$, E. van Duin ${ }^{3}$, B. Veen ${ }^{3}$, M.C.M. van der Salm ${ }^{3}$, A.T.P. van Ittersum ${ }^{4}$, \\ M.L. Bots' ${ }^{2}$, G.E.H.M. Rutten², H.M.M. Vos ${ }^{1}$, M.E. Numans' ${ }^{1}$, R.C. Vos ${ }^{1}$ \\ ${ }^{1}$ Department of Public Health and Primary Care/LUMC-Campus, Leiden University, Leiden, The Netherlands; ${ }^{2} J u l i u s$ Center for Health Sciences and

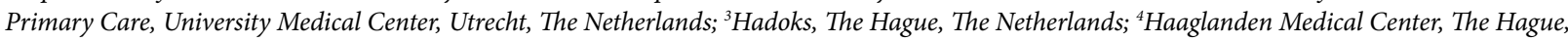 \\ The Netherlands; ${ }^{5} \mathrm{HAGA}$ Hospital, The Hague, The Netherlands \\ E-mail: s.e.van_grondelle@lumc.nl
}

\section{BACKGROUND}

Elevated blood pressure is a common cause for cardiovascular morbidity and mortality. People with elevated blood pressure and type 2 diabetes have an even higher risk on developing cardiovascular complications. An uncontrolled blood pressure despite prescription of blood pressure lowering medication is common. Once people have more chronic conditions, like type 2 diabetes, blood pressure control is only reached in $40-60 \%$. One of the reasons for this is therapy non-adherence. Therapy non-adherence can be addressed on the individual level, the level of the healthcare provider and the healthcare system level. Since less is known about the level of the healthcare providers and the level of the healthcare system, we aimed to explore the facilitators and barriers on these levels in blood pressure care for people with elevated blood pressure with specific focus on therapy adherence.

\section{METHODS}

Qualitative focus group study among healthcare providers in the Hague, The Netherlands. Data were analysed using the theoretical domains framework (TDF) and the behaviour change wheel (BCW).

\section{RESULTS}

The domains 'knowledge', 'interpersonal skills', 'memory, attention and decision process', 'emotions', 'intentions' and 'social influences' of the TDF were found to be relevant on the healthcare provider level. 'Resources' and 'goals' were found to be relevant on the system level. Facilitators in these domains were good interpersonal skills, paying attention to behavioural factors, and the belief that treatment improves health outcomes. Barriers were related to time, interdisciplinary collaboration, technical and financial issues, availability of blood pressure devices and education of people with elevated blood pressure.

\section{CONCLUSION}

This study shows the need for better collaboration between primary and secondary care, more team-based care with pharmacists and social workers, tools to improve interpersonal skills and more time for patient-healthcare provider communication.

\section{Patient preferences regarding education about rotating injection sites}

\author{
Bert Jan Potter van Loon ${ }^{1}$, Prem Adhien ${ }^{2}$, Eric Benning ${ }^{3}$, Alex Muller ${ }^{4}$, Gerben Mol ${ }^{5}$ \\ ${ }^{1} O L V G$, Amsterdam, The Netherlands; ${ }^{2}$ Service Apotheek van Waert, Rotterdam, The Netherlands; ${ }^{3}$ Wilhelmina Ziekenhuis, Assen, The Netherlands; \\ ${ }^{4}$ Diaconessenhuis Utrecht, The Netherlands; ${ }^{5}$ Meander Medisch Centrum, Amersfoort, The Netherlands \\ E-mail: pottervanloon@olvg.nl
}

\section{BACKGROUND}

Lipohypertrophy is found in 20-50 \% of insulin-injecting persons despite extensive coverage on injection technique in guidelines. One of the factors responsible is repetitive injections at the same site. In order to examine what type of education aimed at rotating injection sites would be preferred by persons with diabetes we set out an enquiry.

\section{METHODS}

A questionnaire was handed out to 85 insulin-injecting persons with diabetes mellitus in 5 centres with one open question, 1 qualitative question and 7 yes/no questions.

\section{RESULTS}

85 persons filled in the questionnaire; 55 male and $30 \mathrm{fe}$ male, ages 18-92 years, insulin duration 1-69 years. One person responded having had no education on rotating injection sites; 10 persons responded receiving little education, 62 sufficient and 12 a lot. Yes/No questions: Persons with diabetes valued education by doctor: $68 \%$; education 
cCS bohn bohn
stafleu van loghum

SSS bohn

CL van loghum

SSS bohn

L van loghum

c) bohn stafleu

L van loghum

cer bohn stafleu

L van loghum

ceg bohn bohn
stafleu van loghum

c8h bohn stafleu van loghum

ç bohn stafleu van loghum

c) bohn stafleu van loghum

CS) bohn D) stafleu L. van loghum

cehn bohn stafleu van loghum
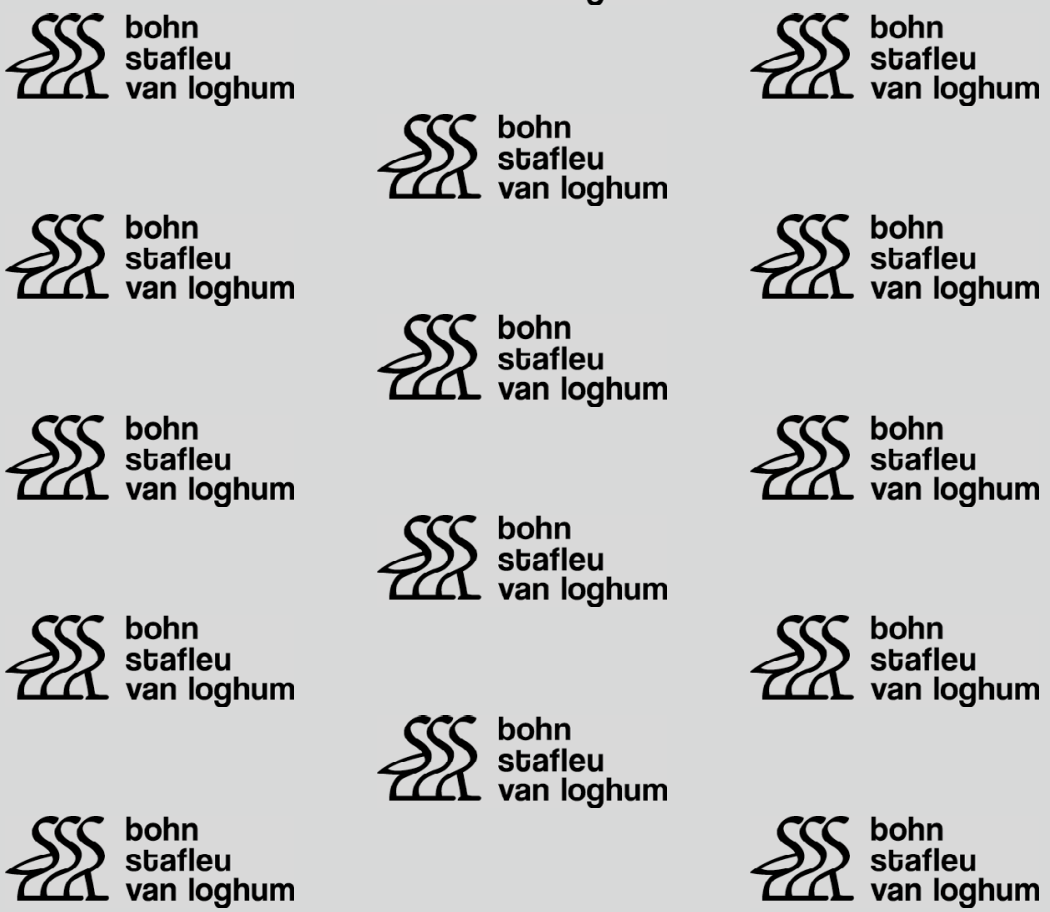

SS lohn

CI van loghum

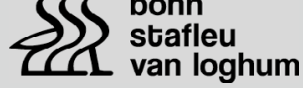

SSS bohn

SSS bohn

CSS bohn stafleu van loghum

SSS bohn stafleu van loghum

SSS bohn

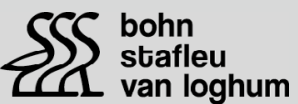

SSS bohn
SSS bohn stafleu van loghum
SSS lohn

SSS bohn

CL van loghum

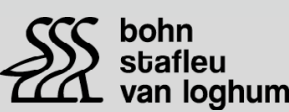

SSS bohn

CL van loghum

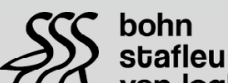

CL $\begin{aligned} & \text { stafleu } \\ & \text { van loghum }\end{aligned}$

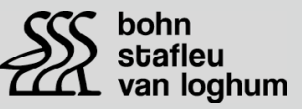

SSS bohn

IL van loghum

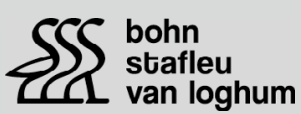

SSS bohn

CL van loghum

SSS lahn $\begin{aligned} & \text { bohn } \\ & \text { vanleu } \\ & \text { van loghum }\end{aligned}$

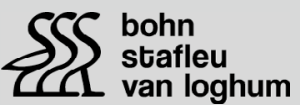

SSS bohn

CL van loghum bohn
stafleu Ll van loghum

SSS bohn II van loghum
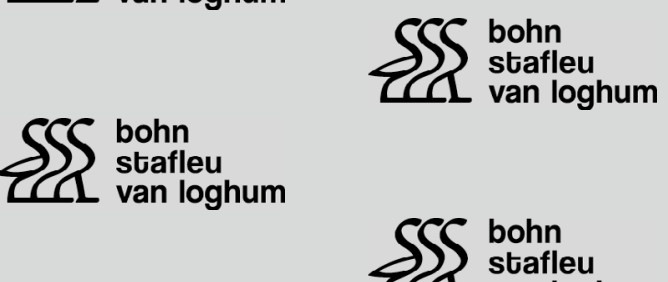
CL van loghum

CI van loghum

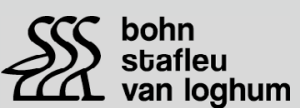

SSS bohn

Cl. van loghum
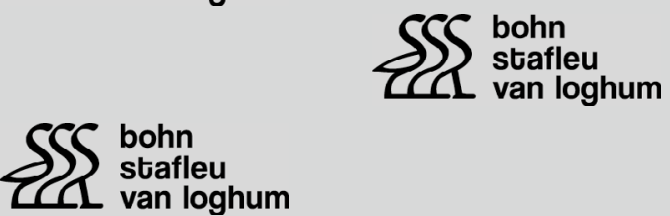

SSS bohn CL van loghum CL van loghum

SSS bohn

CL $\begin{aligned} & \text { stafleu } \\ & \text { van loghum }\end{aligned}$

SSL $\begin{aligned} & \text { bohn } \\ & \text { stafleu } \\ & \text { van loghum }\end{aligned}$

SSS bohn

CL van loghum

SSS bohn

CL van loghum

SSS bohn

CL van loghum

cSc bohn

$\sum$ stafleu

SSl bohn

van loghum

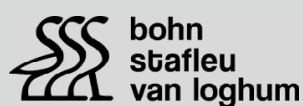

SCS bohn

$\sum \int$ stafleu

L van loghum

SSS bohn

CL van loghum

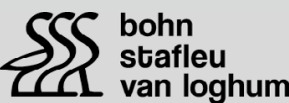

CL $\begin{aligned} & \text { stafleu } \\ & \text { van loghum }\end{aligned}$

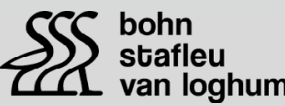

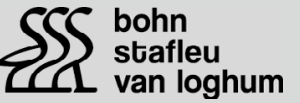
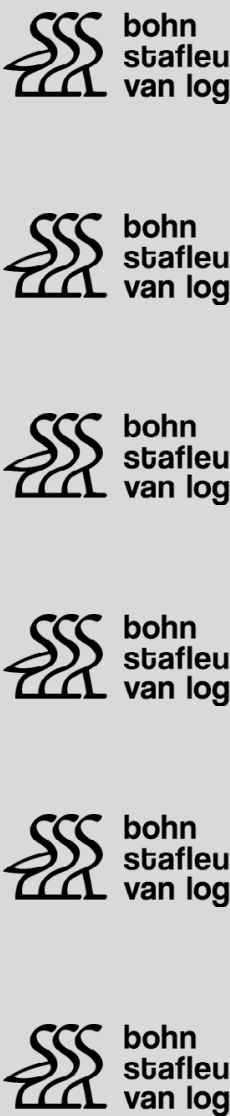

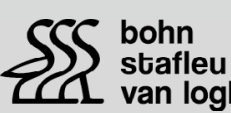

SSS $\begin{aligned} & \text { bohn } \\ & \text { stafleu } \\ & \text { van log }\end{aligned}$

SSS lohn

SSS bohn

ces bohn

CLL van log

L van log
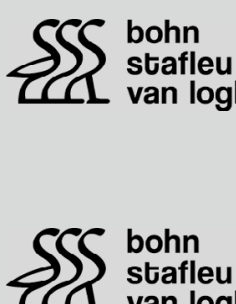

$3 \int \begin{aligned} & \text { bohn } \\ & \text { stafleu } \\ & \text { van log }\end{aligned}$

$\sum S \begin{aligned} & \text { bohn } \\ & \text { stafleu } \\ & \text { van log }\end{aligned}$ 


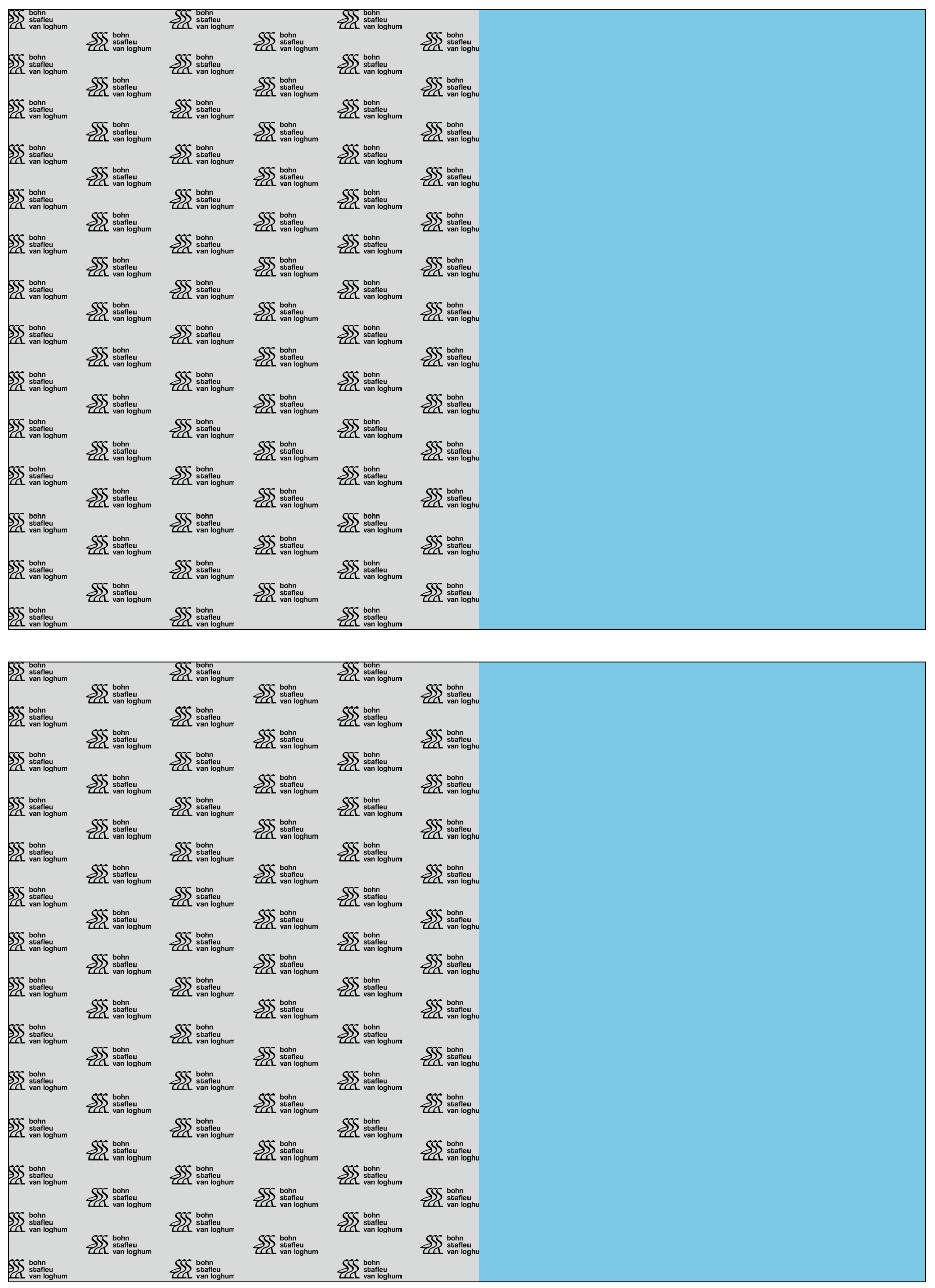


bij diabetes educator: $85 \%$; education by pharmacy: $9 \%$; demonstration of feeling one's own lipohypertrophy: 73\%; receiving a link to YouTube to watch how one can feel one's own lipohypertrophy: 47\%; feeling an artificial lipohypertrophy on a plastic model: $33 \%$ and receiving a pamphlet how to palpate one's own lipohypertrophy: 65\%. 43 out of 85 persons gave a suggestion by answering het open question. There was a preference for visual education of one's own lipohypertrophies (10x); several persons expressed the importance of repeated education $(22 \mathrm{x})$

\section{CONCLUSION}

The majority of insulin-injecting persons with diabetes experienced sufficient education on rotating injection sites; they note the importance of repeated education and prefer conventional educational techniques.

\title{
20
}

\section{Acute vagal nerve stimulation enhances hepatic glucose uptake in anaesthetized rats}

\author{
C.W. Hoornenborg ${ }^{1,2}$, T.H. van Dijk ${ }^{3}$, J.E. Bruggink' ${ }^{1}$, F. Kuipers ${ }^{3,4}$, A.P. van Beek², G. van Dijk ${ }^{1}$ \\ ${ }^{1}$ Department of Behavioral Neuroscience; Groningen Institute for Evolutionary Life Sciences (GELIFES), University of Groningen, Groningen, \\ The Netherlands; Department of ${ }^{2}$ Endocrinology, ${ }^{3}$ Laboratory Medicine and ${ }^{4}$ Pediatrics, University of Groningen, University Medical Center Groningen, \\ Groningen, The Netherlands \\ E-mail:c.w.hoornenborg@rug.nl
}

\section{BACKGROUND}

Modulation of the nervous system through electrical stimulation has allowed the development of treatments to some severe clinical conditions. Among these, vagal nerve stimulation (VNS) has emerged as a new tool to treat obesity and type 2 diabetes (T2DM). Indeed, VNS has previously been shown to cause substantial weight reduction in humans and laboratory animals. However it is not clear if VNS could directly alleviate T2DM or that this is an indirect effect due to the weight loss. Therefore this study uses acute VNS in rats.

\section{METHODS}

After overnight fast, adult male Wistar rats underwent either vagal nerve stimulation (VNS+; $\mathrm{n}=11$ ) or sham stimulation (VNS-; $\mathrm{n}=11$ ) for 120 minutes under anesthesia. Before the stimulation protocol, the rats received a bolus of stable isotope labelled glucose $\left[6,6-{ }_{2} \mathrm{H}_{2}\right]$ glucose. Endogenous glucose production (EGP) and clearance (MCR) were calculated by kinetic analysis from the wash-out of injected D-[6,6-2H2]glucose from the circulation.

\section{RESULTS}

VNS+ resulted in lower glucose levels compared to the VNS- group ( $p<0.05)$, with similar insulin levels. The wash-out of $\mathrm{D}$-[6,6- $\left.{ }^{2} \mathrm{H}_{2}\right]$ glucose was significantly faster in the VNS+ group compared to the VNS- group $(\mathrm{p}<0.01)$. Endogenous glucose production (EGP) and clearance (MCR) by the liver were calculated by kinetic analysis from the wash-out period of injected D-[6,6-2H2]glucose from the circulation. EGP was similar in both groups $(\mathrm{p}=0.06)$ but the MCR was higher in the VNS+ group compared to the VNS- group $(\mathrm{p}<0.001)$.

\section{CONCLUSION}

This study shows that acute VNS has a direct stimulatory effect of hepatic glucose uptake, which is independent of insulin. These effects highlight the potential utilization of VNS as a tool to treat T2DM.

\section{1}

\section{Treatment with Semaglutide $2.4 \mathrm{mg}$ Leads to Improvements in Cardiometabolic Risk Factors in the STEP 1 Trial}

\author{
W. Garvey' ', M. Bhatta, M. Davies, J.E. Deanfield, U. Khalid, M. Kosiborod, R. Kushner, D.M. Rubino, N. Zeuthen, S. Verma, Diederik Smit² (presenting author) \\ ${ }^{1}$ Department of Nutrition Sciences, University of Alabama at Birmingham, Birmingham, USA; ${ }^{2}$ Elisabeth-TweeSteden Ziekenhuis, Tilburg, The Netherlands \\ E-mail: garveyt@uab.edu
}

\section{BACKGROUND}

We evaluated the effect of semaglutide $2.4 \mathrm{mg}$ vs placebo on cardiometabolic risk factors in STEP 1.

\section{METHODS}

STEP 1 (NCT03548935) was a randomised (2:1), dou- 
Table 2. Results on the use of once-weekly semaglutide in patients with type 2 diabetes in SURE Netherlands

\begin{tabular}{|c|c|c|c|c|c|c|}
\hline \multirow[b]{2}{*}{ Parameter } & \multicolumn{2}{|c|}{ Semaglutide $2,4 \mathrm{mg}$ ( $\mathrm{n}=1306$ ) } & \multicolumn{2}{|c|}{ Placebo ( $n=655)$} & \multirow[b]{2}{*}{$\begin{array}{l}\text { Estimated treatment difference } \\
\qquad[95 \% \mathrm{Cl}] \\
\text { (absolute, or estimated treatment } \\
\text { ration expressed as percent) }\end{array}$} & \multirow[b]{2}{*}{ p-value } \\
\hline & Baseline & $\begin{array}{l}\text { Change } \\
\text { (absolute or ratio to } \\
\text { baseline expressend } \\
\text { as percent change) }\end{array}$ & Baseline & $\begin{array}{l}\text { Change } \\
\text { (absolute or ratio to } \\
\text { baseline expressed } \\
\text { as percent change) }\end{array}$ & & \\
\hline Waist circumference, cm & $114,6 \pm 14,8$ & $-13,54$ & $114,8 \pm 14,4$ & $-4,13$ & $-9,42[-10,30,-853]$ & $<0.0001$ \\
\hline Blood pressure, mmHg & $126 \pm 14$ & $-6,16$ & $126 \pm 14$ & $-1,06$ & $-5,10[-6,34,-1,57]$ & $<0.0001$ \\
\hline Systolic & $80 \pm 10$ & $-2,83$ & $80 \pm 10$ & $-0,42$ & & $<0.0001^{*}$ \\
\hline \multicolumn{7}{|l|}{ Diastolic } \\
\hline Lipids, mg/dL & $126,21(47,4)$ & $-22 \%$ & $1267,94(49,0)$ & $-7 \%$ & $-16 \%[-19,-13]$ & $<0.0001^{*}$ \\
\hline Triglycerides & $137,52(27,5)$ & $-6 \%$ & $140,15(25,9)$ & $-1 \%$ & $-6 \%[-8,-4]$ & $<0.0001^{*}$ \\
\hline Non-HDL cholesterol & $110,29(31,6)$ & $-3 \%$ & $112,45(29,8)$ & $+1 \%$ & $-4 \%[-6,-2]$ & $0,0011^{*}$ \\
\hline \multicolumn{7}{|l|}{ LDL cholesterol } \\
\hline CRP, mg/L & $3,87(151,1)$ & $-53 \%$ & $3,87(135,5)$ & $-15 \%$ & $-44 \%[-49,-39]$ & $<0.0001^{*}$ \\
\hline HOMA-IR & $3,02(63,1)$ & $-33 \%$ & $2,99(65,4)$ & $-6 \%$ & $-29 \%[-33,-24]$ & $<0.0001^{*}$ \\
\hline Fasting plasma glucose, mg/dL & $95,4 \pm 10,7$ & $-8,35 \%$ & $94,7 \pm 10,5$ & $-0,48 \%$ & $-7,87[-9,04,-6,70]$ & $<0.0001^{*}$ \\
\hline Fasting serum insulin, mlU/mL & $12,9(58,6)$ & $-26 \%$ & $12,8(61,2)$ & $-7 \%$ & $-21 \%[-26,-17]$ & $<0.0001^{*}$ \\
\hline
\end{tabular}

Results are presented for treatment policy estimand (effect regardless of treatment adherence or use of rescue intervention).

Values are mean \pm standard deviation or geometric mean (coefficient of variation in \%).

The ratio to baseline and the corresponding baseline value were log-transformed prior to analysis.

* Not adjusted for multiplicity

$\mathrm{Cl}=$ confidence interval; $C \mathrm{RP}=$ C-reactive protein; $\mathrm{HDL}=$ high-density lipoprotein; HOMA-IR = Homeostatic Model Assessement of Insulin Resistence; $\mathrm{LDL}=$ low-density

lipoprotein

ble-blind, placebo-controlled trial; 1961 adults with overweight/obesity, without diabetes, received once-weekly subcutaneous semaglutide $2.4 \mathrm{mg}$ or placebo, both plus lifestyle intervention, for 68 weeks. Primary endpoints were percent change in body weight and proportion of subjects achieving $\geq 5 \%$ body weight reduction. Secondary endpoints included change in cardiometabolic risk factors. Post-hoc analyses evaluated change in non high-density lipoprotein (non-HDL) cholesterol and Homeostatic Model Assessment of Insulin Resistance.

\section{RESULTS}

Estimated mean body weight reductions with semaglutide vs placebo were -14.9 vs $-2.4 \%$ (estimated treatment difference [ETD], $-12.44 ; \mathrm{p}<0.0001)$. More subjects achieved $\geq$ $5 \%$ body weight reduction with semaglutide vs placebo (86.4 vs $31.5 \%$; ETD, 11.22; $\mathrm{p}<0.0001$ ). Semaglutide im- proved cardiometabolic risk factors vs placebo: waist circumference $(-13.54$ vs $-4.13 \mathrm{~cm})$, systolic $(-6.16$ vs -1.06 $\mathrm{mmHg})$ and diastolic blood pressure $(-2.83$ vs -0.42 $\mathrm{mmHg}$ ), triglycerides (-22 vs $-7 \%)$, non HDL cholesterol ( -6 vs $-1 \%)$, C reactive protein $(-53$ vs $-15 \%)$ and fasting plasma glucose (-8.35 vs $-0.48 \mathrm{mg} / \mathrm{dL}$ ) (table 2); $\mathrm{p}<0.0001$ for all ETDs/ratios.

\section{CONCLUSION}

Semaglutide $2.4 \mathrm{mg}$ once-weekly treatment for 68 weeks led to superior body weight reductions and greater improvements in cardiometabolic risk factors vs placebo, suggesting favourable cardiometabolic effects of semaglutide beyond body weight loss. 


\title{
Associations among treatment targets, social determinants of health and health care utilisation in Dutch people with type 2 diabetes and mental disorders : results of a linked observational dataset
}

\author{
Emma A. Nieuwenhuijse' ${ }^{1}$, Jeroen N. Struijs ${ }^{1,2}$, Mattijs E. Numans ${ }^{1}$, Rimke C. Vos ${ }^{1}$ \\ ${ }^{1}$ Department of Public Health and Primary Care, Leiden University Medical Center, The Hague, The Netherlands; ${ }^{2}$ National Institute for Public Health and \\ Environment, Bilthoven, The Netherlands \\ E-mail:e.a.nieuwenhuijse@lumc.nl
}

\section{BACKGROUND}

We aimed to determine the impact of mental illness, on reaching diabetes type 2 (T2DM) treatment targets, taking into account effects of health utilisation and social determinants of health (SDOH).

\section{METHODS}

An observational retrospective cross-sectional study based on routine primary care data from The Hague and vicinity, linked to socio-economic and claims data of Statistics Netherlands. Main outcomes were reaching treatment targets for HbAlc, systolic blood pressure (SBP) and LDL-cholesterol in 2017 and were analysed by multivariate logistic regression. Medical determinants included mental illness (based on primary care registration or treated via specialist' mental health care), reimbursed health care costs, medication usage, body mass index, comorbidity and smoking. SDOH included income and Dutch versus non-Dutch origin.

\section{RESULTS}

Overall, 2862 adults (18-80 years) (men: 54.3\%; mean age: 61.8 SD \pm 10.6$)$ were included. Treatment target was reached in 1831 (64.0\%) for HbA1c, 1863 (65.1\%) for SBP and $1516(53.0 \%)$ for LDL-cholesterol. Almost a quarter (22.5\%) had mental illness, of which 323 (50.2\%) received specialist' care. Compared to those without, individuals with mental illness more often were non-Dutch (no mental illness 50.4\%; GP; 56.1\%: specialist care: 59.1\%), had lower income $(46.7 \%, 54.8 \%, 63.2 \%)$ received social benefits $(13.0 \%, 26 \%, 43.5 \%)$ and had higher health care costs (median (IQR) euros: 1851 (946-4153), 2217 (1063-5183), 3036 (1584-6150)). Adjusted for medical determinants and $\mathrm{SDOH}$, mental illness was associated with higher odds on reaching treatment target in adults $<65$ years for $\mathrm{HbAlc}$, (OR (95\% CI): treatment by GP: 1.46 (1.01-2.11); specialist' care: 1.61 (1.11-2.34)). Besides, migration background and income were independent risk factors for HbA1c-target. For SBP mental illness was associated with reaching the treatment target in men only (OR (95\% CI): 1.61 (1.092.40), 1.59 (1.09-2.45)). No association with mental illness was found for reaching LDL-cholesterol treatment targets.

\section{CONCLUSION}

Taking into account $\mathrm{SDOH}$ and health care utilisation, HbA1c targets were more often reached in adults with mental illness aged below 65 years and SBP targets were more often reached in men with mental illness. Mental illness did not affect LDL targets. So, if health care is well delivered to people with both T2DM as mental illness, diabetes control can be achieved, although health care costs are higher and social determinants play an independent role.

\section{3}

\section{Persistent pro-inflammatory effects of a single hypoglycaemic event in humans with and without type 2 diabetes}

\begin{abstract}
Julia I.P. van Heck ${ }^{1}$, Clementine E.M. Verhulst ${ }^{1}$, Therese W. Fabricius ${ }^{2}$, Rinke Stienstra ${ }^{1,3}$, Peter L. Kristensen ${ }^{2,4}$, Cees J. Tack ${ }^{1}$, Ulrik Pedersen-Bjergaard ${ }^{2,4}$, Bastiaan E. de Galan ${ }^{1,5}$ on behalf of the hypo-RESOLVE consortium

${ }^{1}$ Department of Internal Medicine, Radboudumc, Nijmegen, The Netherlands; ${ }^{2}$ Department of Endocrinology and Nephrology, Nordsjoellands Hospital, Hillerød, Denmark; ${ }^{3}$ Division of Human Nutrition and Health, Wageningen University, Wageningen, The Netherlands; ${ }^{4}$ Department of Clinical Medicine, Faculty of Health and Medical Sciences, University of Copenhagen, Denmark; ${ }^{5}$ Department of Internal Medicine, Maastricht University Medical Centre, MUMC+ Maastricht, The Netherlands
\end{abstract}

\section{ACKGROUND}

Hypoglycaemic episodes are the main side-effects of insulin treatment and are associated with increased cardiovascular mortality in patients with diabetes. Activated monocytes defined by enhanced secretion of pro-inflammatory cytokines are key contributors in developing cardiovascular complications. We determined the persistent effects of a single hypoglycaemic event on monocyte phenotype, function and metabolism, and 
circulating inflammatory proteins in humans with type 2 diabetes.

\section{METHODS}

5 adults with and 16 adults without type 2 diabetes (M/F $17 / 14$, age $59.6 \pm 7.1$ years, BMI $\left.28.5 \pm 4.3 \mathrm{~kg} / \mathrm{m}^{2}\right)$ underwent a hyperinsulinaemic-normoglycaemic $(5.3 \pm 0.3$ $\mathrm{mmol} / \mathrm{L})$ hypoglycaemic $(2.8 \pm 0.1 \mathrm{mmol} / \mathrm{L})$ glucose clamp. During euglycaemia, hypoglycaemia, 24 hours, 72 hours and one-week after the hypoglycaemic event blood was drawn to determine ex vivo monocyte phenotype, function and metabolism. Furthermore, 92 circulating inflammatory proteins were determined at all timepoints.

\section{RESULTS}

Hypoglycaemia acutely increased the number of CD16+ monocytes ( $\mathrm{p} \leq 0.001)$ and reduced CD14+ monocytes ( $\mathrm{p} \leq$ 0.001 ). In healthy subjects, this shift persisted up to 72 hours.
Regarding function, hypoglycaemia increased production of TNF- $\alpha$ and IL- $1 \beta(\mathrm{p}<0.05)$, and decreased production of IL-10 $(\mathrm{p}<0.05)$ upon ex vivo stimulation, which normalized after 7 days. Measurements of monocyte glycolysis defining inflammatory potential revealed decreased basal and maximal glycolytic rate during hypoglycaemia $(\mathrm{p}=0.048$ and $\mathrm{p}=$ 0.041 ) that normalized at later timepoints. Hypoglycaemia acutely increased levels of 12 inflammatory proteins $(\mathrm{p}<$ 0.001), such as IL6, IL10 and FGF-21. CSF-1, uPA and Flt3L remained significantly higher up to 7 days $(\mathrm{p}<0.001)$.

\section{CONCLUSION}

One hypoglycaemic event induced pro-inflammatory alterations in monocyte phenotype and function up to 1 week. Besides an acute pro-inflammatory effect, we found persistent inflammation following hypoglycaemia. Our study contributes to unravelling the role of hypoglycaemia in the development of inflammation related complication such as CVD and atherosclerosis.

\title{
24
}

\section{Association between de novo lipogenesis susceptibility genes and coronary artery disease}

\author{
Pomme I.H.G. Simons, Olivier Valkenburg, Coen D.A. Stehouwer, Martijn C.G.J. Brouwers \\ Maastricht University/Maastricht University Medical Centre, Maastricht, The Netherlands \\ E-mail:p.simons@maastrichtuniversity.nl
}

\section{BACKGROUND}

It remains unclear whether de novo lipogenesis (DNL) - a major pathway leading to non-alcoholic fatty liver disease - has a causal effect on the risk of coronary artery disease (CAD). Therefore, this study aimed to assess the association between DNL susceptibility genes and CAD.

\section{METHODS}

DNL susceptibility genes were selected using three approaches: 1) selection of overlapping susceptibility genes for serum triglycerides and sex hormone-binding globulin, both downstream consequences of DNL (unbiased approach), 2) selection of genes based on their known role in DNL (biased approach), and 3) selection of genes that associate with fatty acids, which have previously been used as a proxy of DNL. Gene-CAD effect estimates were retrieved from the meta-analysis of CARDIoGRAM and the UK Biobank including $\sim 76,014$ cases and $\sim 264,785$ controls. Effect estimates were clustered using a fixed-effects meta-analysis. RESULTS
The unbiased approach identified twenty-two DNL susceptibility genes, while the biased approach identified nine, including GCK, GCKR, GPAM, INSR, MLXIPL, PNPLA3, PTEN, SCAP and TRIB1. Seven genes associated with plasma fatty acids. Clustering of genes selected in the unbiased and biased approach showed a statistically significant association with CAD (OR: 1.016, 95\% CI: 1.012 ; 1.020 and OR: 1.013 , 95\% CI: $1.007 ; 1.020$, respectively), while clustering of fatty acid genes did not statistically significantly associate with CAD (OR: 1.004, 95\% CI: 0.996-1.011).

\section{CONCLUSION}

The results suggest that DNL susceptibility genes, but not fatty acid genes, may increase the risk of CAD. These findings warrant further research assessing the beneficial effects of interventions reducing DNL on cardiometabolic disease. 


\title{
The association between social jetlag and (parameters of) the Metabolic Syndrome and Type 2 Diabetes: a systematic review and meta-analysis
}

\author{
Emma J. Bouman ${ }^{1}$, Joline W.J Beulens ${ }^{1,2}$, Lenka Groeneveld ${ }^{1}$, Rozemarijn S. de Kruijk ${ }^{1}$, Linda Schoonmade ${ }^{3}$, Sharon Remmelzwaal ${ }^{1}$, Petra J.M. Elders ${ }^{4}$, \\ Femke Rutters ${ }^{1}$ \\ ${ }^{1}$ Amsterdam UMC, location VUmc, Department of Epidemiology and Data Science, Amsterdam Public Health Research Institute, Amsterdam, \\ The Netherlands; ${ }^{2}$ Julius Centre for Health Sciences and Primary Care, University Medical Centre Utrecht, The Netherlands; ${ }^{3}$ University Library, VU, \\ Amsterdam, The Netherlands; 4Amsterdam UMC, location VUmc, Department of General practice, Amsterdam Public Health Research Institute, \\ Amsterdam, The Netherlands
}

\section{BACKGROUND}

We aimed to determine the association between social jetlag and (parameters of) the Metabolic Syndrome and Type 2 Diabetes (T2D) in a systematic review and meta-analysis.

\section{METHODS}

A systematic literature search was conducted in PubMed/ Embase/Scopus until September 2020. Included studies described an association between social jetlag and (parameters of) the Metabolic Syndrome and/or T2D, were available full text and were written in English or Dutch. Data extraction and quality assessment was performed on pre-piloted forms independently by two reviewers. If three or more studies reported the same outcome, results were meta-analyzed using random-effects analysis.

\section{RESULTS}

5434 titles/abstracts were screened, 133 papers were read full-text, and 46 studies were included. One study was rat- ed as low quality, 22 were moderate quality, and 23 were high methodological quality. The high quality studies showed that having social jetlag compared to no social jetlag was significantly associated with higher levels of BMI $0.39 \mathrm{~kg} / \mathrm{m}^{2}$ (95\% confidence interval $0.01 ; 0.76 ; \mathrm{I}^{2}=100 \%$ ) in 12 studies, higher odds of obesity $1.13\left(1.07 ; 1.18 ; \mathrm{I}^{2}=\right.$ $0 \%)$ in seven studies, higher waist circumference $0.78 \mathrm{~cm}$ $\left(0.22 ; 1.35 ; \mathrm{I}^{2}=7 \%\right)$ in six studies, higher levels of HbA1c $0.65 \%\left(0.12 ; 1.18 ; \mathrm{I}^{2}=100\right)$ in five studies, and lower odds of hypertension $0.56\left(0.17 ; 0.95 ; \mathrm{I}^{2}=53 \%\right)$ in four studies. No statistically significant associations were observed for body fat, abdominal obesity, HDL levels, triglycerides, blood pressure, fasting glucose levels, Metabolic Syndrome or T2D. Sensitivity analyses on health status and statistical quality did not reduce heterogeneity.

\section{CONCLUSION}

Social jetlag is associated with parameters of the Metabolic Syndrome and T2D. However, high heterogeneity for some parameters complicate the interpretation of our results.

\section{6}

\section{ER-mitochondria cross-talk during stress activates innate immune response in beta-cell and contributes to immune cell recruitment}

\author{
Saurabh Vig ${ }^{1}$, Joost M. Lambooij ${ }^{1,2}$, Bruno Guigas ${ }^{2}$, Arnaud Zaldumbide ${ }^{1}$ \\ ${ }^{1}$ Department of Cell and Chemical Biology, Leiden University Medical Center, Leiden, The Netherlands; ${ }^{2}$ Department of Parasitology, Leiden University \\ Medical Center, Leiden, The Netherlands \\ E-mail: S.Vig@lumc.nl
}

\section{BACKGROUND}

Beta-cell destruction in type 1 diabetes (T1D) results from the combined effect of inflammation and recurrent autoimmunity. Accumulating evidence suggest a participation of cellular stress during the initial stage of the disease, preceding destruction and triggering immune cell infiltration. While the role of the endoplasmic reticulum (ER) in this process has been largely described, the participation of the other cellular organelles, particularly the mitochondria that are central mediator for beta-cell survival and function, remains poorly investigated. Here, we have explored the contribution of mitochondria permeability, as consequence of ER stress, in activating the IFN signaling and innate immune response.

\section{METHODS}

Human beta-cell line EndoC- $\beta \mathrm{H} 1$ were exposed to Thapsigargin (TG) or Tunicamycin (TN) to induce ER stress. Seahorse assays were used to analyze mitochondrial fitness and real-time PCR (RT-qPCR) was used to measure the expression of IFN-stimulated genes (ISGs). Cytosolic mitochondria dsDNA (mtDNA) and dsRNA were detected by immunofluo- 
rescence staining and quantified by RT-qPCR. Migration assays were performed to assess neutrophil chemotaxis.

RESULTS

We observed that ER and mitochondrial stress induced by TG and TN correlated with a significant increase expression of ISGs (IFN $\beta(p \leq 0.01)$ and IL8 $(p \leq 0.05))$, a reduced extracellular acidification rate $(\mathrm{p} \leq 0.05)$ and oxygen consumption rate and increased accumulation of cytosolic mtDNA ( $p \leq 0.05$ ) in human beta-cell. We demonstrated that silencing of the cGAS-STING pathway by specific siRNA rescued stress induced ISGs activation and that conditioned medium of stressed beta-cell increased neutrophils migration $(\mathrm{p} \leq 0.05)$.

\section{CONCLUSION}

Our study demonstrates that beta-cell ER stress is associated with mitochondrial dysfunction and increased mtDNA leakage, leading to innate immune response activation via the cGAS-STING pathway. These results provide new mechanistic insights on how ER stress can trigger insulitis and may ultimately allow the identification of potential targets to protect beta-cell from autoimmune destruction.

\title{
27 \\ Clinically-Relevant Weight Loss is Achieved Independently of Early Weight Loss Response to Once-Weekly Subcutaneous Semaglutide 2.4 MG (STEP 4)
}

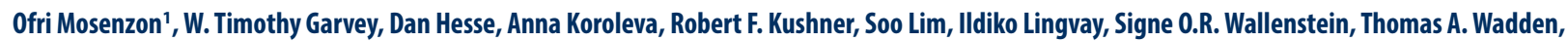 \\ Carel W. Le Roux \\ Presenting author: Diederik Smit ${ }^{2}$ \\ ${ }^{1}$ Diabetes Unit, Department of Endocrinology and Metabolism, Hadassah Medical Center, Faculty of Medicine, Hebrew University of Jerusalem, Israel; \\ ${ }^{2}$ Elisabeth-TweeSteden Ziekenhuis, Tilburg, The Netherlands \\ E-mail: ofrim@hadassah.org.il
}

\section{BACKGROUND}

A post-hoc analysis of the STEP 4 trial (NCT03548987) was conducted to identify whether early weight loss (WL) is predictive of later WL with maintenance once-weekly subcutaneous semaglutide $2.4 \mathrm{mg}$ in people with overweight/obesity.

\section{METHODS}

Adults with $\mathrm{BMI} \geq 27 \mathrm{~kg} / \mathrm{m} 2$ with $\geq 1$ weight-related comorbidity or BMI $\geq 30 \mathrm{~kg} / \mathrm{m} 2$, without T2DM, underwent a 20 -week run-in period with semaglutide $2.4 \mathrm{mg}$. Participants reaching the maintenance dose of once-weekly semaglutide $2.4 \mathrm{mg}$ at week 20 were randomized $2: 1$ to semaglutide $2.4 \mathrm{mg}$ or placebo, including lifestyle intervention, for another 48 weeks. Participants were considered responders if they achieved $\geq 5 \% \mathrm{WL}$ at week 20 . Whether the week 20 response to semaglutide predicted achievement of clinically-relevant WL ( $\geq 5 \%$ ) by week 68 was also assessed.
RESULTS

803 participants were randomized at week 20 (semaglutide: $\mathrm{n}$ $=535$, placebo: $\mathrm{n}=268)$. For responders $(88.0 \%)$ at week 20 , mean body weight change from week 0 to 68 was $-19.7 \%$. For non-responders at week 20 , mean body weight change was $-6.4 \%$ with continued semaglutide vs $-0.3 \%$ with switch to placebo. Of all participants randomized to semaglutide, $86.2 \%$ achieved a clinically-relevant WL $(\geq 5 \%)$ at week 68 . Positive predictive value for responders at week 20 was $96.4 \%$.

CONCLUSION

The majority of participants who were randomized to the maintenance dose of once-weekly semaglutide $2.4 \mathrm{mg}$ at week 20 had lost $\geq 5 \%$ body weight by week 68 , with most achieving this by week 20 . Overall WL with semaglutide was greater among early responders, but non-responders also achieved a clinically-relevant WL by week 68 if semaglutide treatment was continued.

\section{Diet-induced Weight Loss Lowers Plasma Complement C3 via Reduction of Visceral Adipose Tissue: a Randomized Controlled Trial in Abdominally Obese Men}

\author{
Shunxin Jin ${ }^{1}$, Yvo H.A.M. Kusters ${ }^{1,3}$, Alfons J.H.M. Houben ${ }^{1}$, Jogchum Platt ${ }^{2,3}$, Peter J. Joris ${ }^{2,3}$, Ronald P. Mensink ${ }^{2,3}$, Casper G. Schalkwijk ${ }^{1,3}$, \\ Coen D.A. Stehouwer' ${ }^{1}$, Marleen M.J. van Greevenbroek' \\ ${ }^{1}$ Department of Internal Medicine, CARIM, ${ }^{2}$ Department of Nutrition and Movement Sciences, NUTRIM, Maastricht University and Medical Center, \\ Maastricht, The Netherlands; ${ }^{3}$ Top Institute of Food and Nutrition, Wageningen, The Netherlands
}




\section{BACKGROUND}

The alternative complement pathway is identified as risk factor for cardiometabolic diseases. Concentrations of the central complement component C3 and other alternative pathway components are higher in obese persons. We investigated if weight loss affects plasma factor D (also known as adipsin), complement C3, and the alternative pathway anaphylatoxin $\mathrm{C} 3 \mathrm{a}$ and whether these effects, if any, were explained by changes in specific fat depots.

\section{METHODS}

Abdominally obese men $(\mathrm{n}=52,51.8$ years [IQR 45.7; 60.7]) were randomly allocated to a weight stable control group or to an 8-week dietary weight loss intervention. C3, factor D and C3a concentrations were measured in fasting EDTA plasma. Visceral and subcutaneous adipose tissue and intrahepatic lipid (VAT, SAT, IHL, respectively) were measured by MRI. Effect of weight loss was evaluated by one-factor analysis of covariance. Multiple mediator analysis was used to study whether changes in specific fat depots mediated the association of the intervention with changes in complement. Two-tailed $\mathrm{p}<0.05$ was considered statistically significant.

\section{RESULTS}

Diet-induced weight loss was $-10.3 \mathrm{~kg}$ (95\% confidence interval $[-11.4 ;-9.2])$. Changes in fat depots were $-0.81 \mathrm{~L}$ [-0.93;-0.69] for SAT, $-0.85 \mathrm{~L}[-0.10 ;-0.66]$ for VAT and $-0.42 \%$ [-0.57;-0.27] for IHL (all $\mathrm{p}<0.001$ ). Weight loss significantly reduced C3 $(-0.15 \mathrm{~g} / \mathrm{L}[-0.23 ;-0.07], \mathrm{p}<0.001)$, but not factor $\mathrm{D}$ or $\mathrm{C} 3 \mathrm{a}$. Multiple mediation analysis showed that this intervention-induced reduction in C3 (total effect: $-0.117 \mathrm{~g} / \mathrm{L}$ [-0.206;-0.027]) was fully explained by the VAT reduction (mediated effect: $-0.147 \mathrm{~g} / \mathrm{L},[-0.285$;$0.001]$ ) but not by reduction in SAT or IHL.

\section{CONCLUSION}

Diet-induced weight loss in abdominally obese men significantly reduced $\mathrm{C} 3$ concentration, but not factor D or C3a. This weight loss-induced C3 reduction was fully explained by the VAT loss.

\title{
29
}

\section{Cadmium, Active Smoking and Renal Function Deterioration in Patients with Type 2 Diabetes}

\author{
Milou M. Oosterwijk' ${ }^{1}$ Ilse J.M. Hagedoorn' ${ }^{1}$, Ronald G.H.J. Maatman² , Stephan J.L. Bakker ${ }^{3}$ Gozewijn D. Laverman,4 \\ ${ }^{1}$ Department of Internal Medicine/Diabetes, Ziekenhuisgroep Twente, Almelo, The Netherlands; ${ }^{2}$ Clinical Chemistry, Medlon BV, Enschede, \\ The Netherlands; ${ }^{3}$ Department of Internal Medicine/Nephrology, UMCG, Groningen, The Netherlands; ${ }^{4}$ Biomedical Signals and Systems, \\ University of Twente, Enschede, The Netherlands \\ E-mail:mi.oosterwijk@zgt.nl
}

\section{BACKGROUND}

Cadmium is an established nephrotoxin and present in cigarette smoke. We assessed blood cadmium concentrations in active, former and never smokers, and we investigated the hazards of blood cadmium concentration and smoking status on renal function deterioration.

\section{METHODS}

Prospective analyses were performed in data of 226 patients of the DIAbetes and LifEstyle Cohort Twente-1 (DIALECT-1). Cadmium concentrations were determined from EDTA whole blood. Smoking status was collected by a self-administered questionnaire. Renal function deterioration was defined as need for renal replacement therapy or a persistent increase of $\geq 50 \%$ in serum creatinine from baseline visit for at least 3 months. Univariable and multivariable Cox proportional hazards models were used to calculate hazard ratios (HR) for the association between cadmium level, smoking status and renal function deterioration.

\section{RESULTS}

Median [interquartile range] whole blood cadmium level was 2.9 [1.9-5.1] nmol/L. Current smokers had significantly higher cadmium levels (7.4 [3.3-11.7]) compared to patients who never smoked (2.6 [1.6-4.2]) and former smokers (2.8 [1.8-4.8] nmol/L). During median follow-up for 6 [5-8] years, renal function deterioration occurred in 34 persons (15\%). Each doubling of cadmium concentration was associated with an increased hazard for renal function deterioration (HR 1.50, 95\% CI 1.06-2.12); for active smoking the increased HR for renal function deterioration was 5.64 (95\% CI 1.85-17.24). In a multivariable model with both smoking status and cadmium level included, active smokers have an increased hazard for renal function deterioration (HR 4.15, 95\% CI 1.19-14.53) while the association between cadmium and renal function deterioration lost statistical significance $(\mathrm{p}=0.32)$. 
CONCLUSION

Whole blood cadmium level is associated with progressive kidney disease, predominantly determined by smoking behaviour. The main recommendation regarding kidney health is to reduce cadmium exposure via cigarette smoking to a minimum, since the risk of renal function deterioration in patients with T2D might be of concern.

30

\title{
Habitual intake of dietary dicarbonyls is associated with higher insulin sensitivity and lower odds of type 2 diabetes: The Maastricht Study
}

\author{
K. Maasen ${ }^{1}$, S.J.P. Eussen ${ }^{2}$, P.C. Dagnelie ${ }^{2}$, C.D.A. Stehouwer ${ }^{1}$, A. Opperhuizen ${ }^{3}$, M.M.J. van Greevenbroek ${ }^{2}$, C.G. Schalkwijk ${ }^{2}$ \\ ${ }^{1}$ Department of Internal Medicine, School for cardiovascular diseases (CARIM), Maastricht University, The Netherlands; ${ }^{2}$ Department of Epidemiology, \\ Care and Public Health Research Institute (CAPHRI), Maastricht University, The Netherlands; ${ }^{3}$ NUTRIM School of Nutrition and Translational Research \\ in Metabolism, Maastricht University, The Netherlands; Office for Risk Assessment and Research, Food and Consumer Product Safety Authority, Utrecht, \\ The Netherlands \\ E-mail:kim.maasen@maastrichtuniversity.nl
}

\section{BACKGROUND}

Dicarbonyls are major precursors of advanced glycation endproducts (AGEs). Dicarbonyls are formed endogenously and during food processing. Circulating dicarbonyls are positively associated with insulin resistance and type 2 diabetes (T2DM), but consequences of dietary dicarbonyls intake are unknown. Therefore, we examined the associations of dietary dicarbonyls intake with glucose metabolism status (GMS), insulin sensitivity, and beta-cell function.

\section{METHODS}

In 6276 participants ( $60 \pm 9$ yrs, $50 \%$ men, $23 \%$ T2DM) of The Maastricht Study, we estimated habitual intake of the dicarbonyls methylglyoxal (MGO), glyoxal (GO), and 3-deoxyglucosone (3-DG) by linking Food Frequency Questionnaires to our food composition database containing MGO, GO, and 3-DG content of $>200$ foods.

GMS, insulin sensitivity, and beta-cell function were measured by a seven-point oral-glucose-tolerance test. Insulin sensitivity was assessed as Matsuda index (available for $\mathrm{n}=2384)$. Beta-cell function was assessed as C-peptidogenic-index $(n=2381)$. Cross-sectional associations of dietary dicarbonyls with GMS, insulin sensitivity, and beta-cell function were examined using logistic or linear regression, adjusting for age, sex, GMS (except when GMS was outcome), BMI, energy intake, smoking, alcohol, physical activity, education, history of CVD, triglycerides, LDL, cholesterol-to-HDL-ratio, medication, and insulin sensitivity (models with beta-cell function as outcome only).

\section{RESULTS}

Dietary MGO and 3-DG were positively associated with (log-transformed) insulin sensitivity after full adjustment (Std. $\beta[95 \% \mathrm{CI}]=0.07[0.03,0.12]$ and $0.09[0.04,0.13]$, respectively). Dietary MGO, GO, and 3-DG were inversely associated with odds of T2DM after full adjustment (MGO: OR[95\% CI $]=0.77 \quad[0.66,0.90] . \quad$ GO: 0.77 [0.64,0.92]. 3-DG: $0.74[0.61,0.88])$. MGO, GO, and 3-DG intakes were not associated with prediabetes or beta-cell function.

\section{CONCLUSION}

Higher habitual intakes of MGO, GO and 3-DG were associated with lower odds of T2DM. Higher MGO and 3-DG intakes were additionally associated with higher insulin sensitivity. This suggests that food-derived dicarbonyls may play a protective role in T2DM progression. These novel observations require further exploration in prospective cohort and intervention studies.

\section{1}

\section{Pregestational hyperglycemia associates with lower offspring survival rates and accelerates maternal NAFLD development in mouse model for gestational diabetes}

\author{
A.J.C. Tol' ', K. Hribar' ${ }^{1}$, A. De Bruin'², M.H. Oosterveer', E.M. Van der Beek' \\ ${ }^{1}$ Department of Pediatrics, University Medical Center Groningen, Groningen, The Netherlands; ${ }^{2}$ Dutch Molecular Pathology Center, Utrecht University, \\ Utrecht, The Netherlands \\ E-mail:a.j.c.tol@umcg.nl
}




\section{BACKGROUND}

Gestational hyperglycemia either results from pre-existing diabetes (PDM) or develops during pregnancy, the latter referred to as gestational diabetes mellitus (GDM). Both PDM and GDM negatively affect maternal and offspring outcomes. The relation between hyperglycemic exposure and early markers of metabolic dysfunction in the mothers have, however, not been investigated in detail. This study characterized the short-term maternal and offspring consequences of PDM and GDM using a double-hit mouse model.

\section{METHODS}

Female C57BL/6N mice were challenged with a high fat diet (HF) and low-dose streptozotocin (STZ) injections prior to mating to induce gestational hyperglycemia. Controls received HF only. Blood glucose levels at gestational day (GD) 0 and an oral glucose tolerance test (OGTT) at GD15 were used to assess PDM and GDM. Dams and offspring were sacrificed at GD18 or postnatal day (PN)15.
HF-STZ treatment resulted in PDM in $34 \%$ and GDM in $66 \%$ of dams. At GD0, glycemia was comparable in GDM and control dams $(9.6 \pm 1.3 \mathrm{mM}$ vs $8.2 \pm 0.9 \mathrm{mM})$ while PDM dams showed hyperglycemia $(15.5 \pm 1.9 \mathrm{mM}, \mathrm{p}<$ 0.0001). Glucose excursion during OGTT was more severely impaired in PDM as compared to GDM dams (AUC $3035 \pm$ 361 and $2394 \pm 307$ respectively, $\mathrm{p}<0.0001$ ). Maternal PDM, but not GDM, positively correlated to a higher number of resorptions at GD18 $(\mathrm{p}<0.01)$, and reduced litter size $(\mathrm{p}<$ $0.001)$. NAFLD activity score (NAS; $\mathrm{p}<0.05$ ) and plasma ALT $(\mathrm{p}<0.05)$ were increased in PDM dams only. By PN15, GDM dams also showed an increase in NAS (3.6 \pm 1.7 vs 1.2 $\pm 1.0, \mathrm{p}<0.05)$ and hepatic triglyceride content $(102 \pm 25 \mathrm{vs}$ $56 \pm 24 \mathrm{nmol} / \mathrm{mg}, \mathrm{p}<0.01)$ compared to controls.

\section{CONCLUSION}

An earlier onset and more severe hyperglycemia in PDM versus GDM associates with lower offspring survival rates and earlier signs of maternal NAFLD development in mice. Our preclinical approach will be instrumental to dissect the postpartum consequences of GDM and PDM, and to establish the potential of interventions to improve pregnancy and long-term outcomes.

\title{
32
}

\section{Once-weekly semaglutide use in type 2 diabetes: real-world data from the SURE Netherlands observational study}

\author{
Jaap van Soest ${ }^{1}$, Michel P Brugts, Andrei-Mircea Catarig, Alice Clark, Umut Erhan, Aloysius G. Lieverse, Bruce H.R. Wolffenbuttel ${ }^{2}$ \\ ${ }^{1}$ Huisartsenmaatschap MCN, Nijverdal, The Netherlands; ${ }^{2}$ Department of Endocrinology, University of Groningen, University Medical Center Groningen, \\ Groningen, The Netherlands
}

\section{BACKGROUND}

Once-weekly semaglutide demonstrated clinically relevant HbAlc and bodyweight reductions vs comparators in the SUSTAIN randomised controlled type 2 diabetes (T2D) trials. SURE Netherlands (NCT03929679), a multicentre, prospective, non-interventional study, evaluated semaglutide in $\mathrm{T} 2 \mathrm{D}$ routine clinical practice.

\section{METHODS}

Adults ( $\geq 18$ years) with T2D were enrolled. The primary and secondary endpoints were, respectively, change from baseline to end of study (EOS; 30 weeks) in HbAlc and in BW and waist circumference (WC). Proportions of patients achieving predefined $\mathrm{HbAlc}$ targets and weight-loss responses at EOS, safety and patient-reported outcomes were also assessed.

\section{RESULTS}

In total, 211 patients (mean age 60.5 years, diabetes dura- tion 13.3 years) initiated semaglutide; most were receiving metformin $(82.9 \%)$ or basal insulin $(59.2 \%)$ at baseline, and $6.2 \%$ switched from another glucagon-like peptide-1 receptor agonist. In the $186(88.2 \%)$ patients still receiving semaglutide at EOS, treatment was associated with significant improvements in endpoints (table 3). One serious adverse drug reaction (cholecystitis) was reported. Eight patients (all receiving concomitant insulin) experienced severe or documented hypoglycaemia.

\section{CONCLUSION}

Patients with T2D initiating once-weekly semaglutide in routine clinical practice in the Netherlands experienced clinically significant reductions in $\mathrm{HbAlc}$, bodyweight and WC.

Sponsored by Novo Nordisk A/S. 
Table 3. Results on the use of once-weekly semaglutide in patients with type 2 diabetes in SURE Netherlands

\begin{tabular}{|c|c|c|c|c|}
\hline Endpoints measured at baseline and EOS & Baseline value (SD) & Patients analysed, $n$ & $\begin{array}{c}\text { Change from baseline } \\
\text { to EOS (SD) }\end{array}$ & p-value \\
\hline \multicolumn{5}{|l|}{ HbA1C, } \\
\hline$\% / \%$-point & $8.6(1.4)$ & 174 & $-1.2(0.1)$ & $<0.0001$ \\
\hline $\mathrm{mmol} / \mathrm{mol}$ & $70(14)$ & 174 & $-13(0.8)$ & $<0.0001$ \\
\hline Bodyweight, $\mathrm{kg}$ & $105.2(19.0)$ & 160 & $-7.8(0.5)$ & $<0.0001$ \\
\hline Waist circumference, $\mathrm{cm}$ & $119(12)$ & 82 & $-9(0.8)$ & $<0.0001$ \\
\hline \multicolumn{5}{|l|}{ 36-Item Short-Form Health Survey version 2 score } \\
\hline \multicolumn{5}{|l|}{ Physical component summary } \\
\hline \multirow[t]{2}{*}{ Mental component summary } & & 175 & $2.1(0.5)$ & $<0.0001$ \\
\hline & & 175 & $1.9(0.6)$ & 0.002 \\
\hline Diabetes Treatment Satisfaction Questionnaire status version score & $27.9(6.1)^{*}$ & 163 & $3.7(0.3)$ & $<0.0001$ \\
\hline Endpoints measured at EOS only & & & Estimated mean at EOS (SD) & p-value \\
\hline Diabetes Treatment Satisfaction Questionnaire change version score & N/A & 170 & $13.2(0.4)$ & $<0.0001$ \\
\hline Treatment targets/responses at baseline and EOS & $\begin{array}{c}\text { Number at baseline, } \\
\mathbf{n}(\%)\end{array}$ & & Number at EOS, $n(\%)$ & p-value \\
\hline \multicolumn{5}{|l|}{ HbA1C } \\
\hline$<8.0 \%(64 \mathrm{mmol} / \mathrm{mol})$ & $72(34.1)$ & 176 & $124(70.5)$ & NR \\
\hline$<7.5 \%(59 \mathrm{mmol} / \mathrm{mol})$ & $39(18.5)$ & 176 & $95(54.0)$ & NR \\
\hline$<7.0 \%(53 \mathrm{mmol} / \mathrm{mol})$ & $13(6.2)$ & 176 & $65(36.9)$ & NR \\
\hline \multicolumn{5}{|l|}{ Bodyweight reduction } \\
\hline$\geq 3.0 \%$ & $N / A$ & 160 & $123(76.9)$ & NR \\
\hline$\geq 5.0 \%$ & $N / A$ & 160 & $103(64.4)$ & NR \\
\hline $\begin{array}{l}\text { Composite endpoint (reduction in HbATC } \geq 1.0 \% \text {-point and bodyweight } \geq \\
3.0 \% \text { ) }\end{array}$ & $N / A$ & 164 & $73(44.5)$ & NR \\
\hline
\end{tabular}

*Estimated mean. Baseline values $n=211$ (initiated semaglutide), EOS values $n=186$ (still receiving semaglutide at EOS), analysed using an adjusted analysis of covariance model, with change from baseline in the relevant parameter as individual dependent variable. Analysis of primary endpoint adjusted for covariates. EOS, end of study; N/A, not applicable; NR, not reported; SD, standard deviation.

\title{
Distinct associations of plasma methionine and cysteine with fat depots: CODAM and The Maastricht Study
}

\author{
E.C. Tore', M.C.G.J. Brouwers' ${ }^{1}$, P.C. Dagnelie' ${ }^{1}$, A. Elshorbagy' ${ }^{2}$, S.J.P.M. Eussen ${ }^{3}$, J.F.A. Jansen ${ }^{4}$, C.J.H. van der Kallen ${ }^{1}$, M.E. Kooi ${ }^{4}$, S.J.R. Meex ${ }^{5}$, T. Olsen ${ }^{6}$, H. \\ Refsum $^{6}$, C.G. Schalkwijk' ${ }^{1}$, C.D.A. Stehouwer' ${ }^{1}$ K. Vinknes ${ }^{6}$, M.M.J. van Greevenbroek ${ }^{1}$
}

\begin{abstract}
${ }^{1}$ Department of Internal Medicine, CARIM School for Cardiovascular Disease, Maastricht University, Maastricht, The Netherlands; ${ }^{2}$ Department of Pharmacology, University of Oxford, Oxford, United Kingdom; ' ${ }^{3}$ epartment of Epidemiology, CAPHRI Care and Public Health Research Institute, Maastricht University, Maastricht, The Netherlands; ${ }^{4}$ Department of Radiology \& Nuclear Medicine, Maastricht University Medical Center, Maastricht, The Netherlands; ${ }^{5}$ Central Diagnostic Laboratory, Maastricht University Medical Center, Maastricht, The Netherlands; ${ }^{6}$ Department of Nutrition, Institute of Basic Medical Sciences, University of Oslo, Oslo, The Netherlands
\end{abstract}

\section{BACKGROUND}

Sulfur amino acids (SAAs), including the essential amino acid methionine and its derivative cysteine, have been associated with obesity and related metabolic diseases, including type 2 diabetes (T2DM). However, it is not known whether SAAs are related to specific fat depots.

\section{METHODS}

We examined the relationships of fasting methionine and total cysteine, measured in EDTA plasma using LC-MS/ MS, with measures of obesity, body fat distribution and liver fat in two cross-sectional studies enriched with (pre)diabetic individuals: CODAM $(n=470,61.5 \%$ men, $67 \pm 7$ years) and The Maastricht Study (DMS; $\mathrm{n}=371,54.7 \%$ 
men, $61.6 \pm 8.7$ years). Outcomes included BMI, body-fat percentage, waist circumference, trunk subcutaneous or visceral fat (CODAM and DMS); DXA-derived body composition (DMS); ultrasound-derived fatty liver (CODAM) and MRI-derived liver-fat percentage (DMS). Associations were examined with linear or logistic regressions, as appropriate, with $\mathrm{z}$-standardized primary exposures and outcomes. Adjustment was made for relevant confounders related to cohort structure, demographics, lifestyle (including protein intake and physical activity) and in DMS, also for lean mass (not available in CODAM).

\section{RESULTS}

Methionine was associated with fatty liver in CODAM (OR $=1.49,1.19-1.88)$ and liver-fat percentage in DMS $(\beta=0.13$,
0.01-0.26), but not with any measure of obesity. Cysteine was associated with BMI (CODAM: $\beta=0.19 ; 0.09-0.28$; DMS: $\beta$ $=0.11,0.02-0.320)$, body-fat percentage (CODAM: $\beta=0.13$, $0.06-0.19$; DMS: $\beta=0.11,0.40-0.18$ ) and limb fat mass (DMS: $\beta=0.14,0.06-0.23$ ), but not with liver fat.

\section{CONCLUSION}

Methionine and cysteine showed distinct associations with different fat depots, which were similar between studies. Methionine was associated with liver fat, implicated in the development of insulin resistance and T2DM. By contrast, cysteine was associated with BMI and peripheral fat depot. These results call for further investigation on the role of SAAs in regional fat distribution, and their relationship with T2DM.

\title{
34
}

\section{The glycolytic by-product methylglyoxal is present in very high concentrations in leukocytes and increases after an oral glucose tolerance test.}

\author{
Xiaodi Zhang ${ }^{1}$, Jaycey Kelly ${ }^{1}$, Jean Scheijen ${ }^{1}$, Matthew Streeter ${ }^{2}$, David Spiegel ${ }^{2}$, Casper Schalkwijk ${ }^{1}$, Kristiaan Wouters ${ }^{1}$ \\ ${ }^{1}$ Department of Internal Medicine, School for Cardiovascular Diseases (CARIM), Maastricht University, Maastricht, the Netherlands; ${ }^{2}$ Department of \\ Chemistry, Yale University, New Haven, United States
}

\section{BACKGROUND}

Immune cell activation has been identified as a key driver of diabetic complications. Activation of immune cells is linked with increased glycolysis. The dicarbonyl compound methylglyoxal (MGO) is formed as a byproduct of glycolysis. Plasma MGO levels peak after a glucose-load in individuals with diabetes. It is currently unknown what the levels of MGO are in circulating immune cell subsets and whether MGO accumulates in these cells after a glucose load. We therefore investigated levels and accumulation of MGO in circulating immune cells.

\section{METHODS}

MGO was quantified in human blood fractions using ultra-performance liquid chromatography-tandem mass spectrometry and in lymphocytes, granulocytes, and monocytes isolated from healthy donors using fluorescence-activated cell sorting (FACS). Potential peroxidase interference and consequent $\mathrm{MGO}$ formation during pre-analytical procedure was avoided by performing the derivatization directly in perchloric acid at a low $\mathrm{pH}$. Next, we estimated MGO content in the specific circulating immune cell subsets with flow cytometry using a fluorescent probe that detects MGO. MGO concentrations are present- ed as mean \pm standard deviation.

\section{RESULTS}

MGO levels were much higher in whole blood than in plas$\mathrm{ma}(3.31 \pm 0.54 \mu \mathrm{M}$ vs $0.076 \pm 0.023 \mu \mathrm{M})$. Leukocyte and erythrocyte fractions contained comparable amounts of MGO and contributed to the majority of whole blood MGO. Further measurements in purified leukocyte subsets showed that intracellular MGO concentrations were extremely high in lymphocytes $(2698 \pm 1888 \mu \mathrm{M})$, granulocytes $(1706 \pm 925 \mu \mathrm{M})$, and monocytes $(2873 \pm 427 \mu \mathrm{M})$. Intracellular MGO concentrations were 1000 -fold lower in erythrocytes $(2.53 \pm 1.46 \mu \mathrm{M})$. During an oral glucose tolerance test, the glucose load increased intracellular MGO levels in granulocytes with $6.5 \%$, in monocytes with $18 \%$ and in lymphocytes with $26 \%$.

\section{CONCLUSION}

MGO is present in a very high concentration in leukocytes and is increased in leukocytes after a glucose load in abdominally obese individuals. 


\title{
Development of a new Phosphorus Magnetic Resonance Technique to detect NAD-metabolites in skeletal muscle
}

\author{
Julian Mevenkamp ${ }^{1}$, Yvonne M.H. Bruls ${ }^{1,2}$, Robin A. de Graaf ${ }^{3}$, Joachim E. Wildberger ${ }^{1}$, Matthijs K.C. Hesselink², Patrick Schrauwen ${ }^{2}$, L. Lindeboom ${ }^{1,2}$, \\ Vera B. Schrauwen-Hinderling ${ }^{1,2}$ \\ ${ }^{1}$ Departments of Radiology and Nuclear Medicine, NUTRIM School for Nutrition and Translational Research in Metabolism, Maastricht University \\ Medical Center, Maastricht, The Netherlands; ${ }^{2}$ Department of Nutrition and Movement Sciences, NUTRIM School for Nutrition and Translational \\ Research in Metabolism, Maastricht University Medical Center, Maastricht, The Netherlands; ${ }^{3}$ Department of Radiology and Biomedical Imaging, Yale \\ School of Medicine, New Haven, USA
}

\section{BACKGROUND}

Nicotinamide adenosine dinucleotide (NAD) plays an important role in energy metabolism. NAD+ forms a redox couple with NADH and a high concentration of $\mathrm{NAD}^{+}$as well as a high $\mathrm{NAD}^{+} / \mathrm{NADH}$ ratio are strongly associated with metabolic health. In contrast, decreased $\mathrm{NAD}^{+}$bioavailability is reported in both ageing and obese humans as well as in diabetic mice. Currently, assessment of NAD metabolites in muscle relies on (invasive) biopsies. Determination of NAD metabolites using standard ${ }^{31} \mathrm{P}$-MRS is not possible in muscle, since the resonance of a-ATP is overlapping and thereby masking the NAD resonances. Here, we developed a non-invasive phosphorus magnetic resonance spectroscopy $\left({ }^{31} \mathrm{P}-\mathrm{MRS}\right)$ technique to determine NAD metabolites in vivo. The newly developed MRS sequence was validated in vivo by measuring the $\mathrm{NAD}^{+} / \mathrm{H}$ levels in resting state and during ischemia, as it is well known that ischemia decrease $\mathrm{NAD}^{+} / \mathrm{NADH}$ levels.

\section{METHODS}

We applied a homonuclear BIRD (HB) filter to suppress the $\alpha$-ATP resonance, allowing quantification of $\mathrm{NAD}^{+} /$ $\mathrm{NADH}$ (figure 1). For validation, we included 8 young healthy lean participants and $\mathrm{NAD}^{+} / \mathrm{NADH}$ were measured in the lower leg in rest and during eight minutes of ischemia using this new ${ }^{31} \mathrm{P}-\mathrm{MRS}$ technique. Ischemia was induced by inflating an upper leg cuff to a pressure of 50 $\mathrm{mmHg}$ above systolic blood pressure.

\section{RESULTS}

The HB filter succeeded in suppressing the a-ATP resonance by $85 \%$ and allowed quantification of $\mathrm{NAD}^{+} /$
Figure 1. Mean total scores (maximum score is 105) of the GSRS questionnaire, divided per group and dose.

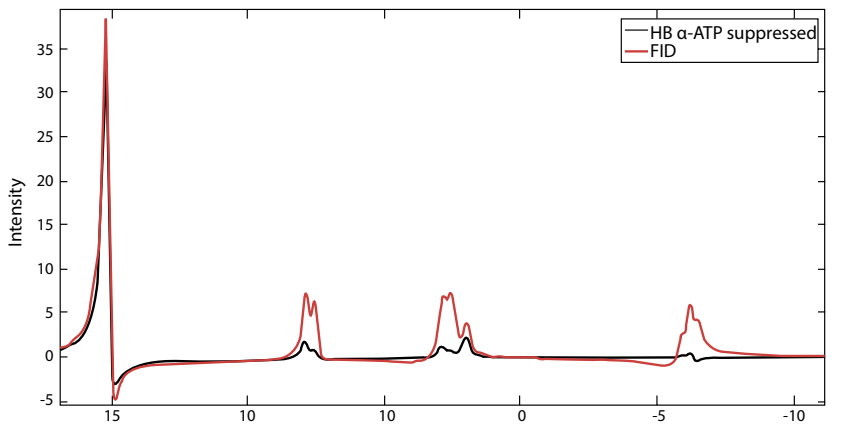

MRS FID acquisitions with (black line) and without (red line) homonuclear BIRD filter of an ATP + NAD phantom. a-ATP is at approximately $3.0 \mathrm{ppm}$ and NAD at $2.7 \mathrm{ppm}$ in the acquired spectra. An $75 \%$ reduction of the a-ATP resonance by the homonuclear BIRD filter can be observed. Signal loss due to $T_{2}$ effects of the filter reduces NAD amplitude by ca. 30\%

NADH levels. Validation measurements with acute ischemia revealed an acute reduction in $\mathrm{NAD}^{+}$signal intensity (from $93 \pm 4$ to $78 \pm 4$ [AU], $\mathrm{p}<0.05$ ) and tended to reduce the ratio $\mathrm{NAD}^{+} / \mathrm{NADH}$ (from $3.41 \pm 0.71$ to $2.21 \pm 0.29, \mathrm{p}=0.08$ ), in line with previous research in muscle biopsies.

\section{CONCLUSION}

We developed a state-of-the-art ${ }^{31} \mathrm{P}-\mathrm{MRS}$ technique enabling non-invasive quantification of NAD metabolites. Applying this technique during ischemia, we could detect the expected changes in $\mathrm{NAD}^{+}$and $\mathrm{NADH}$, showing that physiological changes in NAD metabolites can be detected by dedicated ${ }^{31} \mathrm{P}$-MRS. This provides new non-invasive means for future metabolic research on the relevance of NAD metabolism in type 2 diabetes.

\section{6 \\ Kidney Function Measures and Cardiovascular Outcomes in People with Diabetes: the Hoorn Diabetes Care System cohort}

\author{
E. Dal Canto ${ }^{1,2}$, A. A. van der Heijden ${ }^{1}$, A.J. van Ballegooijen' ${ }^{1}$, B.I. Lissenberg-Witte' ${ }^{1}$, F. Rutters' ${ }^{1}$ P. Elders ${ }^{2}$, J.W.J. Beulens ${ }^{1,3}$
}

${ }^{1}$ Department of Epidemiology and Data Science, Amsterdam University Medical Center, Amsterdam, The Netherlands; ${ }^{2}$ Department of General Practice and Elderly Care Medicine, Amsterdam University Medical Center, Amsterdam, The Netherlands; ${ }^{3} J u l i u s$ Center for Health Sciences and Primary Care, University Medical Center Utrecht, Utrecht, The Netherlands 


\section{BACKGROUND}

Both estimated glomerular filtration rate (eGFR) and urinary albumin-to-creatinine ratio (UACR) are strongly associated to cardiovascular disease (CVD) risk in adults with diabetes. It is however unclear whether each manifestation of kidney disease in diabetes could contribute to increase the risk of specific CVD subtypes. Accordingly, we aimed to assess the prospective association between longitudinally repeated measures of eGFR and UACR and the occurrence of myocardial infarction (MI), coronary heart disease (CHD), stroke, heart failure (HF) and cardiovascular mortality in adults with diabetes.

\section{METHODS}

13,657 patients with T2D from The Hoorn Diabetes Care System cohort, a population-based study, were followed-up annually since 1998. Multivariate time-dependent Cox regression models adjusted for common cardiovascular risk factors, were used to estimate hazard ratios (HRs) and 95\% CI.

\section{RESULTS}

among the 13,657 patients $(53.6 \%$ males, mean[SD] age 62.3 [12.1] years), during a median follow-up time of 7 years (interquartile range 3-12) event rates per 1000 per- son-years were 3.08 for MI, 3.72 for CHD, 1.12 for HF, 0.84 for stroke and 6.25 for cardiovascular mortality. Moderately (60-90) and severely reduced eGFR $(<59)$ was prospectively and independently associated with a higher risk of MI $(\mathrm{HR}=1.53,95 \% \mathrm{CI}: 1.10 ; 2.13$ and $\mathrm{HR}=1.80,95 \% \mathrm{CI}$ : $1.15 ; 2.81)$, $\mathrm{CHD}(\mathrm{HR}=1.68,95 \% \mathrm{CI}: 1.24 ; 2.28$ and $\mathrm{HR}=$ $2.05,95 \% \mathrm{CI}: 1.37 ; 3.08)$ and stroke $(\mathrm{HR}=2.54,95 \% \mathrm{CI}$ : $1.28 ; 5.05$ and $\mathrm{HR}=2.51,95 \% \mathrm{CI}: 1.00 ; 6.30)$, compared to eGFR $>90 \mathrm{~mL} / \mathrm{min} / 1.73 \mathrm{~m} 2$. Conversely, moderately (330) and severely increased ( $>30$ ) UACR was prospectively associated with a higher risk of cardiovascular mortality in men and women ( $\mathrm{HR}=1.99,95 \% \mathrm{CI}: 1.50 ; 2.63$ and $\mathrm{HR}=$ $3.16,95 \%$ CI: 2.03 ; 4.92) and with a higher risk of HF in women only $(\mathrm{HR}=2.78,95 \% \mathrm{CI}: 1.50 ; 5.20)$, compared to $\mathrm{UACR}<3 \mathrm{mg} / \mathrm{mmol}$.

\section{CONCLUSION}

This study showed that a longitudinal decline of eGFR associated to a higher risk of atherosclerotic CVD, while increased UACR associated with higher risk of HF and cardiovascular mortality. The observed differential associations between each manifestation of kidney disease and CVD suggest a regular monitoring of kidney function over time could help identifying diabetes patients at higher risk of specific cardiovascular events.

\title{
37
}

\section{Momentary Assessment of Type 1 Diabetes patient's Experiences in Glucose Variability and Mood in Real life (MERITS): First findings}

\author{
M. de Wit, E. Serné, D. van Raalte, F.J. Snoek \\ Amsterdam UMC, Amsterdam, The Netherlands \\ E-mail:m.dewit@amsterdamumc.nl
}

\section{BACKGROUND}

Blood glucose excursions are commonly reported as a source of diabetes-distress. Research is inconclusive about the associations between momentary glucose parameters and mood. We aimed to assess the relationship between real-time glucose variability and glycemic control with current mood in adults with type 1 diabetes, taking into
Figure 2. Mean total diarrhea scores (maximum score is 21) of the GSRS questionnaire, divided per group and dose.

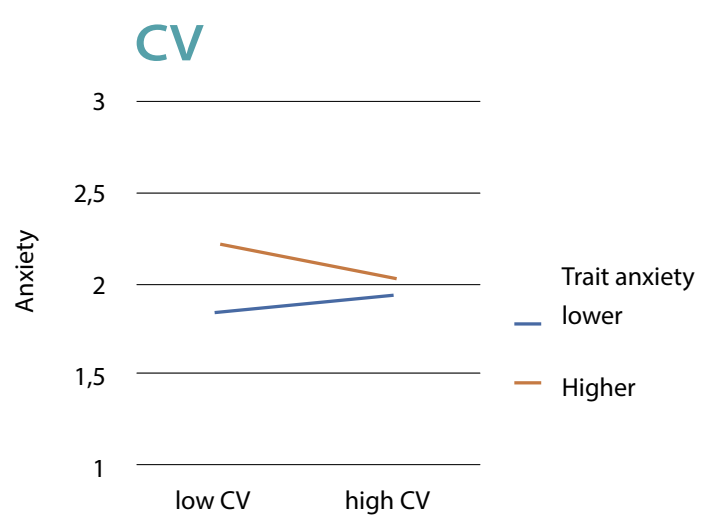

CV*GAD-7 : Estimate $=.0 .122, \mathrm{p}<0.001$

\section{Time below Range}

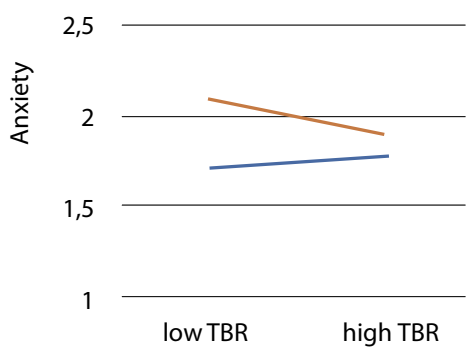

TBR $^{*}$ GAD-7 : Estimate $=.0 .11, \mathrm{p}<0.05$ 
account inter-individual differences.

\section{METHODS}

For 2 weeks, participants wore a blinded CGM and received a questionnaire on their smartphones 6 times a day about their current mood (Profile Of Mood States (POMS; Anxiety, Depressive mood, Anger, Fatigue, Vigor). Mixed model analyses examined associations between glucose parameters within one day (Covariance (CV), Time In Range ( $\geq 3.9$ TIR $\leq 10 \mathrm{mmol} / \mathrm{l})$, Time Above Range (TAR, $>10 \mathrm{mmol} / \mathrm{l})$ and Time Below Range (TBR, < $3.9 \mathrm{mmol} / \mathrm{l})$ ) and POMS dimensions. Interaction terms with baseline trait anxiety (GAD-7), emotional well-being (WHO-5), fatigue (CIS-8) and impaired awareness of hypoglycemia (IAH; Gold $\geq 4$ ) examined inter-individual differences.
$\mathrm{N}=22$ participants. Trends showed a higher CV to be associated with more Fatigue, and more TIR with more vigor, less depressive mood and less anger (all p $<0.1$ ). CV and TBR were significantly associated with POMS anxiety with an interaction with the GAD-7 score (CV: $p=0.001$; TBR: $\mathrm{p}=0.03$ ): Only for those with higher trait anxiety at baseline, lower $\mathrm{CV}$ and less TBR were associated with higher momentary anxiety (figure 2). Increased TAR was associated with more momentary anxiety, especially in those with higher trait anxiety at baseline $(\mathrm{p}<0.05)$ and with IAH $(\mathrm{p}<0.05)$.

\section{CONCLUSION}

Our findings show a complex interaction of glucose parameters and mood with inter-individual differences. The direction of the association between real-time mood and different glucose parameters warrants further investigation.

\title{
38 \\ Immunoproteasome expression correlates with beta cell immunogenicity and accelerates processing of DRiP epitopes
}

\author{
Sofia Thomaidou' ${ }^{\text {, Amadeo Munoz Garcia }}{ }^{2}$, Arno van der Slik ${ }^{1}$, Bart 0. Roep ${ }^{2}$, Francoise Carlotti and Arnaud Zaldumbide $^{1}$ \\ ${ }^{1}$ Department of Cell and Chemical Biology, ${ }^{2}$ Department of Internal Medicine, Leiden University Medical Center, Leiden, The Netherlands \\ E-mail: s.thomaidou@lumc.nl
}

\section{ACKGROUND}

In autoimmune disease, impaired thymic education or low affinity T-cells are believed to be responsible of the immune attack directed against native self-proteins. Yet, there is increasing evidence that local inflammation or other forms of stress combined with genetic predisposition leads to the generation and accumulation of aberrant or modified proteins to which central tolerance is lacking. We and others have shown that these neo epitopes can be generated through disturbance of the transcriptional, post-transcriptional/translational processes. Similar to transcription and translation, protein degradation is not a static process and can be regulated in response to extracellular signals to ensure homeostasis, survival or apoptosis. In tumors, several specific epitopes were shown to be derived from the immunoproteasome or its intermediate conformations and not by constitutive proteasome. Learning from tumor immunology, we evaluated the participation of the immunoproteasome to islet immunogenicity in T1D.

\section{METHODS}

We use single cell RNA sequencing to characterize changes in the antigen processing machinery in human beta cells and evaluate peptide presentation to specific T1D patient-derived CD8 T cells directed against insulin defective ribosomal product (Kracht et al. Nat Med 2017) after IFN $\gamma$ and IFNa stimulation.

\section{RESULTS}

Single cell seq RNA analyses performed on primary human islet cells exposed to proinflammatory cytokines revealed upregulation of genes involved in antigen processing and in particular genes encoding the catalytic subunits of the immunoproteasome. We confirmed gene and protein expression of PSMB8, PSMB9 and PSMB10 in human beta cell line and primary human islets after exposure to inflammatory cytokines and demonstrated that specific knock down of the PSMB8 subunit by siRNA limits processing and presentation of insulin DRiP to specific CD8 T cells.

\section{CONCLUSION}

Our data demonstrate the importance of the degradation machinery in beta cell immunogenicity and position the immunoproteasome catalytic subunits as potential therapeutic targets to limit neoantigens processing and beta cell destruction. 


\title{
Effectiveness of a stepped-care approach versus immediate continuous glucose monitoring-based technologies in hypoglycaemia-prone patients with type 1 diabetes (ECSPECT-HYPO)
}

\author{
E.H. Serné, D.H. van Raalte, M. de Wit, F.J. Snoek \\ Amsterdam UMC, Amsterdam, The Netherlands
}

\section{BACKGROUND}

Guidelines suggest a stepped-care approach in the management of patients with impaired awareness of hypoglycaemia (IAH), initially with structured psycho-educational programs based on Blood Glucose Awareness Training (BGAT), progressing to diabetes technology in those with persisting need. We examined the clinical effectiveness of a stepped-care approach starting with HypoAware (adaptation of BGAT) and adding continuous glucose monitoring (CGM) as needed, versus immediate CGM in type 1 diabetes patients with IAH (Goldscore $\geq 4$ ).

\section{METHODS}

A pragmatic prospective, parallel-group, multicentre, cluster-randomised controlled trial. The intervention group attended HypoAware. If IAH was still present after 6 months or a severe hypoglycaemic event (SHE) had occurred, CGM was offered. Primary endpoint was the number of participants with self-reported SHE. Secondary outcomes, evaluated with mixed-model-analyses at 6 and 12 months, were $\mathrm{HbA1c}$, the number of participants with $\mathrm{IAH}$, time in clinically significant hypoglycaemia $(<3.0 \mathrm{mmol} / \mathrm{L} ; \mathrm{TCSH})$.

\section{RESULTS}

At 6 months, the number of patients reporting SHE had decreased significantly more in the CGM group compared to the stepped-care group:-63\% $(-14 / 22 ; \mathrm{n}=0$ medical assistance $[\mathrm{MA}])$ vs $-27 \%(-8 / 30 ; 4 \mathrm{MA})(\mathrm{p}<0.05)$. HbAlc decreased more in the CGM group compared to the stepped-care group $(-0.37$ percentage points $[-0.49$ to $-0.25], \mathrm{p}<0.05)$. The number of patients without IAH increased in both groups $(+33 \%$ vs $+32 \%)$. TCSH was higher in the stepped-care group compared to the CGM group (3.0 (0.2-9.4)\% vs $0.6(0-1.8) \%$; $\mathrm{p}<0.05)$. In the steppedcare group $\mathrm{n}=17$ started CGM, $\mathrm{n}=11$ started intermittently scanned CGM (isCGM), and $\mathrm{n}=2$ patients started neither CGM nor isCGM. At 12 months the number of patients reporting SHE was still higher in the stepped-care group compared to the CGM group ( $\mathrm{p}<0.05)$, especially after subsequent start of isCGM compared to CGM.

\section{CONCLUSION}

In individuals with type 1 diabetes with IAH and a high risk of SHE immediate start of CGM is more effective in reducing SHE risk. Subsequent start of isCGM in the stepped care group seems less effective compared to the start of CGM.

\section{ECG abnormalities and major adverse cardiac events in people with type 2 diabetes: The Hoorn Diabetes Care System cohort}

Peter P. Harms' ${ }^{1}$, Petra. J.M. Elders, Femke Rutters, Birgit I. Lissenberg-Witte, Hanno L. Tan, Joline W.J. Beulens, Giel Nijpels, Amber A. van der Heijden for ESCAPE-NET

${ }^{1}$ Amsterdam UMC, The Netherlands

E-mail:p.p.harms@amsterdamumc.nl

\section{BACKGROUND}

dentifying early signs of cardiovascular disease in people with type 2 diabetes (T2D) is important, because timely treatment may prevent complications. Electrocardiogram (ECG) abnormalities are common in people with T2D without symptoms of cardiovascular disease, and can be early signs of impending major adverse cardiac events (MACE). We aimed to investigate the association of (changes in) six types of ECG abnormalities with incident MACE, in people with T2D without pre-existing cardiovascular disease.

\section{METHODS}

A prospective study of 11,993 people with T2D without known cardiovascular disease from the Hoorn Diabetes Care System cohort. Annually repeated measurements (1998-2018) included cardiovascular risk factors, an ECG, and self-reported cardiovascular events. ECG abnormalities were classified according to the Minnesota Classification as prolonged PR duration, prolonged QRS duration, left QRS-axis, QS pattern, ST-segment/T-wave abnormalities, or tall R-wave. The association of ECG abnormalities with MACEs was assessed using time-dependent Cox-regression models, adjusted for cardiovas- 
cular risk factors and medication use (Hazzard Ratios with 95\%CIs).

\section{RESULTS}

During a median follow-up of 6.6 (IQR, 3.1-10.7) years, 5445 (45.4\%) of the participants had an ECG abnormality (prevalent or incident) at any of the median 6 (IQR, 3-10) annual ECG recordings, and 905 people (7.5\%) had a MACE (529 coronary heart disease, 250 heart failure, 126 were sudden cardiac arrest). After adjustment, prolonged QRS duration (HR, 2.12 (95\%CI, 1.66-2.70)), QS pattern (2.85 (1.47-5.52)), ST-segment/T-wave abnormalities (2.44
(1.83-3.26)), and tall R-wave (1.38 (1.01-1.89)), were associated with MACE, predominantly heart failure. The associations were not modified by age, sex, hypertension or estimated cardiovascular disease risk.

\section{CONCLUSION}

In people with $\mathrm{T} 2 \mathrm{D}$ without pre-existing cardiovascular disease, ECG abnormalities are early signs of emerging cardiovascular disease that convey an up to twofold increased risk of MACE, specifically heart failure. All people with T2D should be periodically screened for cardiovascular disease with an ECG.

\title{
41
}

\section{Markers of residual beta-cell function are associated with more time in euglycaemic range in type 1 diabetes}

\author{
Coco M. Fuhri Snethlage ${ }^{1}$, Elena Rampanelli' , Bart 0. Roep ${ }^{2}$, Catherine B. Brouwer ${ }^{3}$, Suat Simsek ${ }^{4}$, Daniël H. van Raalte ${ }^{1}$, Max Nieuwdorp ${ }^{1}$, \\ Nordin M.J. Hanssen ${ }^{1}$ \\ ${ }^{1}$ Amsterdam Diabetes Centrum, Amsterdam UMC, location AMC/VUmc, Amsterdam, The Netherlands; ${ }^{2}$ City of Hope National Medical Center, \\ Los Angeles, USA; ${ }^{3}$ Onze Lieve Vrouwen Gasthuis, location Oost, Amsterdam, The Netherlands; ${ }^{4}$ Noord West Ziekenhuisgroep, Alkmaar, The Netherlands \\ E-mail: c.m.fuhrisnethlage@amsterdamumc.nl
}

\section{BACKGROUND}

Preservation of residual beta-cell function in type 1 diabetes (T1D) is known to reduce long term complications. It is conceivable this is due to improved daily glucose control, but the association between residual beta-cell function and improved continuous glycaemic control is unknown. We investigated whether residual beta-cell function is associated with more time spent in euglycaemic range in $\mathrm{T} 1 \mathrm{D}$.

\section{METHODS}

146 individuals with T1D were included in this cross-sectional study (age $41 \pm 15$ years, $58 \%$ female, HbAlc: $53 \pm 12$ $\mathrm{mmol} / \mathrm{mol}$, diabetes duration median 12 years (interquartile range (IQR): 5-27years)). Fasting plasma C-peptide concentrations $(n=146)$ and postprandial urinary C-peptide/creatinine ratios (subset of $n=76$ ) were measured as markers of residual beta-cell function. Time in euglycaemic range (glucose concentration of $3.9-10 \mathrm{mmol} / \mathrm{L}$ ) and hypoglycaemic range $(<3.9 \mathrm{mmol} / \mathrm{L})$ were monitored 14 days prior to the study visit, with a continuous glucose monitoring device. Spearman correlations were calculated in SPSS version 27.0.

\section{RESULTS}

Median fasting plasma C-peptide concentrations were 0.05 $\mathrm{nmol} / \mathrm{mmol}$ (IQR: $0.05-0.13$ ) and the median postprandial urinary C-peptide/creatinine ratio was 0.03 (IQR: 0.00$0.88) \mathrm{nmol} / \mathrm{mmol}$. Higher fasting plasma C-peptide concentrations correlated with more time in euglycaemic range ( $\mathrm{r}=0.459, \mathrm{p}=0.001)$, and lower mean glucose concentrations $(r=-0.320, p=0.001)$ and less time in hypoglycaemic range $(r=-0.443, p=0.001)$. In addition, higher postprandial urinary $\mathrm{C}$-peptide/creatinine ratios correlated with more time in euglycaemic range $(r=0.443, p=0.001)$, lower mean glucose $(\mathrm{r}=-0.318, \mathrm{p}=0.005)$ and less time in hypoglycaemic range $(r=-0.325, p=0.004)$. Results remained overall similar in linear regression models adjusted for sex, age, plasma glucose and duration of diabetes.

\section{CONCLUSION}

Both plasma and urinary beta-cell function markers correlated with more time in euglycaemic range and less time in hypoglycaemic range in individuals with T1D. Beneficial effects of beta-cell preservation in T1D may therefore be attributable to less intermittent glucotoxicity and hypoglycaemic episodes. Acknowledgments: This research was supported by a DNF DON grant 2020, number 2020.10.002. 


\title{
Gestational Diabetes Mellitus perturbes offspring glucose excursion at young adult age
}

\author{
K. Hribar' ${ }^{1}$, E.M. van der Beek ${ }^{1}$, M.H. Oosterveer ${ }^{1}$ \\ ${ }^{1}$ Department of Pediatrics, University Medical Center Groningen, Groningen, The Netherlands \\ E-mail:k.hribar@umcg.nl
}

\section{BACKGROUND}

Gestational diabetes mellitus (GDM) increases the risk for the mother and child to develop obesity and type 2 diabetes (T2D) in later life. To define preventive or curative therapies, the course and mechanisms underyling T2D development in GDM offspring need to be established. We wanted to assess glucose homeostasis in young adult offspring born from GDM pregnancies.

\section{METHODS}

Female C57BL/6N mice were challenged with a high fat diet (HF) and low-dose streptozotocin (STZ) injections prior to mating to induce GDM. Control dams received HF or low fat (LF) diets. Glucose excursion in female and male offspring was assessed around postnatal day 100 (PN100) using oral glucose tolerance (OGTT) and meal tolerance tests (MTT). A separate cohort of female offspring was mated around postnatal day 95 and subjected to an OGTT during gestational day 14 (GD14) of pregnancy.

\section{RESULTS}

showed earlier and higher OGTT peak blood glucose levels compared to LF and HF controls (14.9 vs $12.4 \mathrm{mM}$; p = 0.0014 ), while male GDM and HF offspring showed higher OGTT peak glucose levels compared to LF offspring (15.2 and 15.0 vs $11.5 \mathrm{mM} ; \mathrm{p}=0.0003$ and $\mathrm{p}=0.0150)$. Similar, but more modest effects on glucose excursion were observed during the MTTs in both sexes. GDM Females 13.9 vs LF $11.6 \mathrm{mM}(\mathrm{p}=0.0249)$ and GDM Males at 14.8 vs $11.8 \mathrm{mM}$ $(\mathrm{p}=0.0191)$. Body weight and adipose tissue mass were comparable in GDM and control offspring of both sexes at PN95. Pregnant female GDM offspring showed impaired glucose tolerance compared to LF and HF controls (14.1 vs 13.0 and $12.1 \mathrm{mM})$.

\section{CONCLUSION}

Exposure to maternal GDM induces modest and distinct changes in glucose excursion in male and female mouse offspring at young adult age. These findings indicate a programming effect induced by gestational hyperglycemia and mark an early stage of metabolic disease development in GDM offspring.

Fasting glycemia did not differ. Female GDM offspring

43

\section{A novel in vitro model of metabolically-activated human macrophages for studying adipocyte-macrophage cross-talk during obesity}

\author{
Joost M. Lambooij ${ }^{1,2}$, Juliet Schaapherder ${ }^{1}$, Julia Bremser ${ }^{1}$, Frank 0 tto $^{1}$, Arnaud Zaldumbide ${ }^{2}$, Bruno Guigas ${ }^{1}$ \\ Departments of ${ }^{1}$ Parasitology, and ${ }^{2}$ Cell and Chemical Biology, Leiden University Medical Center, Leiden, The Netherlands \\ E-mail: j.m.lambooij@lumc.nl
}

\section{BACKGROUND}

Macrophages are highly plastic myeloid cells present as resident cells in many tissues or recruited upon inflammatory stimuli. Adipose tissue macrophages (ATMs) play a central role in metabolic homeostasis and obesity-induced changes in their polarization are mainly driven by tissue microenvironment. Macrophage polarization has historically been divided into pro- (M1) and anti-inflammatory (M2), but recent studies showed that obesity promotes a specific, metabolically-activated ATM phenotype that differs from this M1/M2 paradigm. Here we aimed to develop an in vitro model of human macrophages that more closely resembles the obesity-induced ATMs.

\section{METHODS}

Monocytes isolated from human PBMCs were differentiated towards macrophages using M-CSF and stimulated with a mixture of saturated- and unsaturated fatty acids in presence or not of insulin and glucose for 24 hours. Macrophage phenotype was assessed by cell-surface marker expression (flow-cytometry), gene expression (qPCR), and cellular metabolic rates (Seahorse).

\section{RESULTS}

Lipid stimulation induced a dose-dependent increase in cell-surface expression of CD40 $(\mathrm{p}=0.06)$ and CD86 $(\mathrm{p} \leq$ 0.05 ) and decrease in CD209 and CD206 ( $\leq 0.05)$. In addi- 
tion, a significant increase in the gene expression of the secreted pro-inflammatory factors IL- $1 \beta$, CCL2 and TNFa ( $p$ $\leq 0.05)$ and decrease in the expression of IL-10 ( $\mathrm{p} \leq 0.05)$ were observed, a phenotype resembling M1-like macrophages. However, we also found an increase in the expression of genes related to metabolic activation, such as CD9, Plin2, LAMP1 and SPP1 (all p $\leq 0.05$ ), indicating a distinct activation compared to M1-like macrophages. Lipid-stimulated cells also displayed increased oxidative phosphorylation and glycolysis ( $\mathrm{p} \leq 0.05)$, confirming an overall increase in cellular metabolism. These changes were unaffected by concomitant exposure to insulin and glucose.

\section{CONCLUSION}

Our study establishes a new in vitro model of metabolically-activated human monocyte-derived macrophages that may be considered more physiologically relevant for studying macrophage-adipocyte cross-talk in the context of obesity. Additional studies are ongoing to further characterize the transcriptomic signature, secretome and phagocytic functions of these peculiar macrophages.

\title{
44 \\ Three weeks of time restricted eating improves fasting glucose in type 2 diabetes patients but does neither increase nocturnal fat oxidation nor insulin sensitivity
}

\author{
C. Andriessen, C. Fealy, A. Veelen, K. Roumans, E. Kornips, N. Connell, J. Hoeks, P. Schrauwen \\ Department of Nutrition and Movement Sciences, Maastricht University, Maastricht, The Netherlands
}

\section{BACKGROUND}

Time restricted eating (TRE) is a novel strategy to improve metabolic health by limiting the daily eating time window and consequently prolonging the overnight fast. This prolonged fast increases energy storage utilization and might improve insulin sensitivity due to an increased need to replenish nutrient storages. Here we examined if 3 weeks of TRE could improve nocturnal fat oxidation and metabolic health of overweight/obese adults with T2D.

\section{METHODS}

In a randomized controlled cross-over trial, $14 \mathrm{~T} 2 \mathrm{D}$ patients were instructed to eat either within $10 \mathrm{hrs}$ during daytime (TRE) or to spread food intake over at least $14 \mathrm{hrs}$ per day (CON), for 3 weeks, without altering habitual energy intake. Outcome parameters included nocturnal energy expenditure and substrate oxidation assessed by wholeroom calorimetry, insulin sensitivity measured by a 2 -step hyperinsulinemic-euglycemic clamp, and fasting blood metabolites.

\section{RESULTS}

Patients lost more weight after 3 weeks TRE compared to the weight-stable CON $(-1.0 \pm 1.1 \mathrm{~kg}$ vs $-0.3 \pm 1.0 \mathrm{~kg}, \mathrm{p}<0.05)$. Fasting glucose levels were lower after TRE compared to CON ( $7.6 \pm 1.3 \mathrm{mmol} / \mathrm{l}$ vs $8.6 \pm 1.5 \mathrm{mmol} / \mathrm{l}, \mathrm{p}<0.05)$. Sleeping metabolic rate tended to be lower in TRE compared to CON $(4.66 \pm 0.51 \mathrm{~kJ} / \mathrm{min}$ vs $4.77 \pm 0.66 \mathrm{~kJ} / \mathrm{min}, \mathrm{p}=0.06)$ without concomitant changes in substrate utilization. Peripheral insulin sensitivity did not differ between TRE and CON (rate of disappearance: $20.0 \pm 5.6 \mu \mathrm{mol} / \mathrm{kg} / \mathrm{min}$ vs. $19.2 \pm 5.9 \mu \mathrm{mol} / \mathrm{kg} / \mathrm{min}$, respectively; $\mathrm{p}=0.59$ ).

\section{CONCLUSION}

Three weeks of TRE improved fasting glucose levels and decreased body weight in T2D patients. These effects were not accompanied by changes in peripheral insulin sensitivity and/or changes in nocturnal substrate oxidation. These data suggest that TRE may hold promise for long-term glucose homeostasis in type 2 diabetes patients but further research is needed.

\section{5}

\section{Novel biomarkers for glycaemic deterioration in type 2 diabetes: an IMI-RHAPSODY study}

\begin{abstract}
Roderick C. Slieker, Louise A. Donnelly, Livia Lopez-Noriega, Giuseppe N. Giordano, Mikael Åkerlund, Alexander Efanov, Valeriya Lyssenko, Cristina Legido Quigley, Leif Groop, Mark Ibberson, Joline W.J. Beulens, Leen M. 't Hart, Ewan R. Pearson, Guy A. Rutter
\end{abstract}

\section{IMI-RHAPSODY}

\section{BACKGROUND}

Type 2 diabetes is a progressive multifactorial disease which presently affects $>400 \mathrm{~m}$ worldwide, with numbers expected to increase to $>700 \mathrm{~m}$ by 2045. Biomarkers for the disease, which provide a deeper understanding of the disease process, are therefore eagerly sought. 


\section{METHODS}

We have deployed a multi-omics approach in large cohorts of patients with existing type 2 diabetes to identify biomarkers for disease progression across three molecular classes, metabolites, lipids and proteins. A Cox regression analysis for association with time to insulin requirement in 2,973 patients in the DCS, ANDIS and GoDARTS cohorts identified homocitrulline, isoleucine and 2-aminoadipic acid, as well as the bile acids glycocholic and taurocholic acids, as predictive of more rapid deterioration. Increased levels of eight triacylglycerol species, and lowered levels of the sphingomyelin SM 42:2;2 were also predictive of disease progression.

\section{RESULTS}

Of $\sim 1,300$ proteins examined in two cohorts, levels of GDF15/MIC1, IL-18RA, CRELD1, NogoR, FAS, and ENPP7 were associated with faster progression, whilst SMAC/DIABLO, COTL1, SPOCK1 and HEMK2 predicted lower progression rates. Strikingly, identified proteins and lipids were also associated with diabetes incidence and prevalence in external replication cohorts. Implicating roles in disease compensation, NogoR/RTN4R improved glucose tolerance in high fat-fed mice and tended to improved insulin signalling in liver cells whilst IL-18R antagonised inflammatory IL-18 signalling towards nuclear factor kappa-B in vitro. Conversely, high NogoR levels led to islet cell apoptosis.

\section{CONCLUSION}

This comprehensive, multidisciplinary approach thus identifies novel biomarkers with potential prognostic utility, provides evidence for new disease mechanisms, and identifies potential therapeutic avenues to slow diabetes progression.

\title{
46
}

\section{Single-cell RNA sequencing of human pancreatic islets reveals a role of pancreatic duct cells as mediator of the inflammation during the early stage of T1D}

\author{
Amadeo Muñoz García, Nathalie Groen, Floris Leenders, Arnaud Zaldumbide, Eelco de Koning, Françoise Carlotti
}

Department of Internal Medicine, LUMC, Leiden, The Netherlands

E-mail:a.munoz_garcia@lumc.nl

\section{BACKGROUND}

Type 1 diabetes (T1D) is an inflammatory disease that is characterized by the autoreactive destruction of pancreatic beta-cells by immune cells. The goal of this study is to investigate the response of human pancreatic cells to pathophysiological conditions associated with T1D, ultimately to unravel the molecular mechanisms driving loss of the functional beta-cell mass in T1D.

\section{METHODS}

Pancreatic islets from 3 non-diabetic donors were treated with inflammatory stressors (IL1 $\beta$ \& IFN $\gamma$ and IFNa) for 24 and $72 \mathrm{~h}$ to mimic the physiopathology scenarios that occurs in diabetes. After treatment, islets were processed for single-cell RNA sequencing. In addition, islets from 2 T1D donors and 3 non-diabetic controls were processed for single-cell RNA-seq. Finally, validation experiments were performed with human islets and EndoCBH1 cells treated with human recombinant IL8 or blocking IL8 antibody.

\section{RESULTS}

Proinflammatory conditions significantly compromised beta-cell identity and function. A subpopulation of beta-cells (HMOX1+,DDIT3+, SQSTM1+,SLC3A2+) presented lower levels of MHCs when exposed to IL1 $\beta$ \& IFN $\gamma$ treatment. Anti-oxidant defense is increased in islet cells, while duct cells show a clear pro-inflammatory profile. We confirmed the presence of a duct subpopulation presenting a proinflammatory signature in T1D, including increased expression of IL8. Furthermore, we show that IL8 activates NFkB in beta-cells. IL8 treatment compromises primary human beta-cell function, while blocking IL8 prevents cytokine-induced beta-cell failure.

\section{CONCLUSION}

We identified a beta-cell subpopulation that presents an adaptation to an inflammation environment that may represent a protective mechanism to T1D by lowering antigen-presentation capacity, and thereby reducing the risk of recognition by (auto-)immune cells. Furthermore, our data revealed a potential role of the duct cell compartment in the amplification of islet inflammation in T1D. Finally, we show that the pro-inflammatory cytokine IL8 can act directly on beta-cells and have a detrimental effect on beta-cell function. 


\section{Context of physical activity matters for risk of type 2 diabetes}

Jeroen van der Velde ${ }^{1}$, Esther Winters-van Eekelen ${ }^{1}$, Frits Rosendaal ${ }^{1}$, Renée de Mutsert ${ }^{1}$

${ }^{1}$ Department of Clinical Epidemiology, LUMC, Leiden, The Netherlands

E-mail: jeroen.vandervelde@lumc.nl

\section{BACKGROUND}

Physical activity is important in the prevention and treatment of type 2 diabetes. However, differential health effects have been reported depending on the context in which physical activity is performed. Therefore, we examined the risk of type 2 diabetes for different contexts of physical activity.

\section{METHODS}

In the Netherlands Epidemiology of Obesity study, context of physical activity (in METh/week): occupational (during

\section{RESULTS}

We analysed data of 3,766 participants (53\% men) with a mean (SD) BMI of $29.6(4.6) \mathrm{kg} / \mathrm{m}^{2}$ ), aged 54 (5) years. During a median follow-up of 6.7 years, 178 incident cases of type 2 diabetes were reported. Leisure time physical activity (HR: 0.66 [0.43-1.01] in highest quartile) and commuting physical activity (HR: 0.58 [0.370.91 ] in highest quartile) were associated with decreased risk for type 2 diabetes. Occupational physical activity was not associated with decreased risk for type 2 diabetes (table 4).

Table 4. risk of type 2 diabetes (hazard ratio (HR) and 95\% confidence intervals (CI)) for quartiles of occupational, leisure time, commute, and household physical activity

\begin{tabular}{|c|c|c|c|c|c|}
\hline & & Q 1 (lowest) & Q2 & Q3 & Q4 (highest) \\
\hline & & HR (95\% Cl) & HR (95\% Cl) & HR (95\% Cl) & HR (95\% Cl) \\
\hline \multirow[t]{2}{*}{ Occupational PA } & Adjusted model & 1 & $0.93(0.60-1.44)$ & $0.93(0.62-1.39)$ & $1.37(0.89-2.10)$ \\
\hline & + total body fat & 1 & $0.97(0.62-1.50)$ & $0.95(0.64-1.42)$ & $1.24(0.80-1.90)$ \\
\hline \multirow[t]{2}{*}{ Leisure time PA } & Adjusted model & 1 & $0.78(0.53-1.15)$ & $0.63(0.42-0.95)$ & $0.58(0.36-0.86)$ \\
\hline & + total body fat & 1 & $0.84(0.58-1.24)$ & $0.69(0.45-1.03)$ & $0.66(0.43-1.01)$ \\
\hline \multirow[t]{2}{*}{ Commute PA } & Adjusted model & $1^{*}$ & $0.82(0.57-1.18)$ & $0.49(0.31-0.77)$ & \\
\hline & + total body fat & $1^{*}$ & $0.87(0.61-1.26)$ & $0.58(0.37-0.91)$ & \\
\hline \multirow[t]{2}{*}{ Household PA } & Adjusted model & 1 & $0.57(0.36-0.89)$ & $0.81(0.53-1.24)$ & $0.87(0.54-1.41)$ \\
\hline & + total body fat & 1 & $0.58(0.37-0.90)$ & 0.88 (0.58-1.34) & $0.87(0.54-1.40)$ \\
\hline
\end{tabular}

$\mathrm{PA}=$ physical activity. Associations were adjusted for age, sex, ethnicity, educational background, Dutch healthy index, and smoking. ${ }^{*} 01$ and $\mathrm{Q} 2$ were combined due to number of participants who reported no commute PA.

work), commute (walking or cycling to work), leisure time, and household, was assessed at baseline by questionnaire. Incidence of type 2 diabetes was collected during 10 years of follow-up via electronic health records. In adults working $\geq 12 \mathrm{~h} /$ week, we calculated hazard ratios (HR) with $95 \%$ confidence intervals [CI] of type 2 diabetes for quartiles of context of physical activity using Cox regression analyses (with lowest quartile as reference). Results were adjusted for demographic and lifestyle variables and total body fat.

\section{CONCLUSION}

In middle-aged men and women, physical activity performed during commute or leisure time was associated with a decreased risk of type 2 diabetes, whereas occupational physical activity was not. Consequently, people with high occupational physical activity should be physically active apart from work-related physical activity. 


\title{
In patients with obesity, higher levels of psychological stress are associated with lower levels of the insulin-sensitizer FGF21
}

\author{
Susanne Kuckuck*1 ${ }^{\text {, Robin Lengton*1 }}{ }^{*}$ Eline S. van der Valk ${ }^{1}$, Anand M. Iyer ${ }^{1}$, Mostafa Mohseni ${ }^{1}$, Sjoerd A.A. van den Berg ${ }^{1,2}$, Elisabeth F.C. van Rossum ${ }^{1}$ \\ *Shared first authorship \\ ${ }^{1}$ Department of Internal Medicine, Division of Endocrinology, Erasmus MC, University Medical Center Rotterdam, Rotterdam, The Netherlands; \\ ${ }^{2}$ Department of Clinical Chemistry, Erasmus MC, Rotterdam, The Netherlands
}

\section{BACKGROUND}

An intact hormonal regulation of appetite is crucial for preventing weight gain in a world full of obesogenic temptations. Disrupted signaling of hormonal appetite regulators is often observed in individuals with obesity $\left(\mathrm{BMI} \geq 30 \mathrm{~kg} / \mathrm{m}^{2}\right)$ and related diseases, such as type 2 diabetes. Previous research indicates that such disturbances may be induced by weight gain itself, but also other factors such as glucocorticoid excess (e.g. due to stress). However, knowledge regarding the associations between hormonal appetite signals and biological or psychological stress measures is still limited.

\section{METHODS}

Data were collected from 67 patients with obesity of which $21 \%$ had type 2 diabetes. Psychological stress was assessed with the Perceived Stress Scale (PSS)-14 and biological stress was measured using the average of $24 \mathrm{~h}$ urine cortisol levels of two consecutive days. Moreover, levels of hormonal appetite regulators (leptin, adiponectin, insulin, FGF21, PP, GIP, PYY, CCK, AgRP and resistin) were measured in serum after an overnight fast. The cross-sectional associations between psy- chological/biological stress and hormonal appetite regulators were studied by means of linear regression analysis adjusted for pre-defined potential confounders (age, sex and BMI).

\section{RESULTS}

Most hormonal appetite regulators were not associated with PSS-14 or $24 \mathrm{~h}$ urine cortisol levels. However, there was a negative association between FGF21 and PSS-14 ( $\mathrm{r}=$ $-.312, \mathrm{p}=.019)$ which persisted after multivariable adjustment for the potential confounders.

\section{CONCLUSION}

In patients with obesity, higher levels of psychological stress are associated with lower levels of the insulin-sensitizer FGF21, independently of age, sex and BMI. In view of previous evidence showing that glucocorticoids reduce circulating levels of FGF21, we hypothesize that (long-term) psychological stress may interfere with FGF21 signaling via increased HPA-axis activity. Future studies should investigate the directionality of this relation and its potential role in the development of type 2 diabetes.

\section{9}

\section{Sex disparities in treatment transitions after metformin initiation among patients with type 2 diabetes}

\author{
Monika P. Oktora' ${ }^{1}$, Stijn de Vos, Sieta T. de Vries, Eelko Hak, Petra Denig ${ }^{1}$ \\ ${ }^{1}$ Department of Clinical Pharmacy and Pharmacology, University of Groningen, University Medical Center Groningen, Groningen, The Netherlands \\ E-mail:m.p.oktora@umcg.nl
}

\section{BACKGROUND}

Although guidelines for the treatment of diabetes recommend the same stepwise treatment for men and women, some disparities in prescribed medication have been observed. This study aims to assess sex differences among patients with type 2 diabetes (T2D) in 1) treatment transitions after metformin initiation, 2) time between metformin initiation and treatment intensification, and 3) $\mathrm{HbAlc} \mathrm{level} \mathrm{at} \mathrm{initiation} \mathrm{and} \mathrm{intensification.}$

\section{METHODS}

A cohort study was conducted using the Groningen Initia- tive to ANalyze Type 2 diabetes Treatment (GIANTT) database which contains electronic medical records from a dynamic cohort of T2D patients from the north of the Netherlands. Patients aged $\geq 18$ years who initiated metformin in the period 2004-2012, and had a follow-up period of 2-5 years were included. Markov models were developed to estimate transition probabilities. Chi-square tests were used to compare differences between sexes in the transition probabilities. Significant differences between sexes were measured using $\mathrm{p}$ value $<0.05$. Kaplan-Meier analyses were used to assess the time to event between metformin initiation to treatment intensification. HbA1c levels between the sexes were compared using Mann-Whitney U tests. 
1,374 days). Men had on average a higher HbA1c level at initiation (7.3 vs 7.1) and intensification (7.6 vs 7.5), most-

The most common transition after metformin initiation was a dose increase (55\%). Men received more often intensification, i.e. metformin dose increase, addition of another oral hypoglycemic-agent (OHA), and re-initiation of metformin after discontinuation. Women more often continued on metformin monotherapy, discontinued metformin, and initiated another OHA after discontinuation. The time between metformin initiation and treatment intensification did not differ between men and women $(1,363$ vs ly when they received additional OHA with/without higher metformin dose (7.8 vs 7.6).

\title{
CONCLUSION
}

The observed sex disparities in treatment transitions among T2D patients after metformin initiation suggest that men may be more in need of treatment intensification, whereas women may have more problems with tolerating metformin.

\section{0}

\section{The association of serum magnesium with cardiovascular complications in type 1 and 2 diabetes}

\author{
Lynette J. Oost ${ }^{1}$, Amber A.W.A. van der Heijden² ${ }^{2}$ Cees J. Tack ${ }^{3}$, Joline W.J. Beulens ${ }^{4}$, Jeroen H.F. de Baaij ${ }^{1}$
}

${ }^{1}$ Department of Physiology, Radboud Institute for Molecular Life Sciences, Radboud University Medical Center, Nijmegen, The Netherlands; ${ }^{2}$ Department of General Practice and Elderly Care Medicine, Amsterdam UMC - location VUmc, Amsterdam, The Netherlands; ${ }^{3}$ Department of Internal Medicine, Radboud Institute for Molecular Life Sciences, Radboud University Medical Center, Nijmegen, The Netherlands; ${ }^{4}$ Department of Epidemiology \& Data Science, Amsterdam Public Health, Amsterdam Cardiovascular Sciences research institutes Amsterdam UMC - location VUmc, Amsterdam, The Netherlands

\section{BACKGROUND}

We examined the prevalence of hypomagnesemia (magnesium blood levels $<0.7 \mathrm{mmol} / \mathrm{L}$ ) and the correlation of serum magnesium levels in individuals with type 1 and $2 \mathrm{di}-$ abetes.

\section{METHODS}

Serum $\mathrm{Mg}^{2+}$ was measured in two independent cohorts; the Diabetes Care System (DCS) ( $\mathrm{n}=4,348$ type 2 diabetes (T2D)) and DM300 $(\mathrm{n}=241$ type 1 diabetes (T1D)). We analysed whether serum $\mathrm{Mg}^{2+}$ was correlated with clinical data, laboratory measurements and macro- and microvascular complications using linear regression models.

RESULTS

In individuals with $\mathrm{T} 2 \mathrm{D}$, the average serum $\mathrm{Mg}^{2+}$ concentration was $0.80 \pm 0.08 \mathrm{mmol} / \mathrm{L}$, while $9.1 \%$ of the participants had hypomagnesemia. The prevalence of hypomagnesemia in individuals with T1D was much lower; only
$2.9 \%$ had hypomagnesemia with a mean serum $\mathrm{Mg}^{2+}$ of $0.84 \pm 0.10 \mathrm{mmol} / \mathrm{L}$. In T2D, serum $\mathrm{Mg}^{2+}$ is inversely associated with poor glycemic control, while in T1D this is limited to individuals that are insulin resistant. Serum $\mathrm{Mg}^{2+}$ was, prospectively, inversely, associated with heart failure (HF) 0.76 (95\% CI: 0.62; 0.93), atrial fibrillation (AF) 0.59 (95\% CI: $0.49 ; 0.72)$ and overall microvascular complications 0.85 (95\% CI: 0.78; 0.91) (including chronic kidney disease 0.89 (95\% CI: $0.82 ; 0.96$ ), diabetic retinopathy 0.77 (95\% CI: $0.61 ; 0.98)$ and diabetic foot 0.85 (95\% CI: 0.78 ; $0.92)$ in $\mathrm{T} 2 \mathrm{D}$. There was no association of serum $\mathrm{Mg}^{2+}$ with cardiovascular complications in individuals with T1D.

\section{CONCLUSION}

Serum $\mathrm{Mg}^{2+}$ is, inversely, associated with glycaemic control and the risk to develop HF, AF and the occurrence of microvascular endpoints, $\mathrm{CKD}$, diabetic retinopathy and foot complications, in T2D. The association of serum $\mathrm{Mg}^{2+}$ with glycaemic control is limited to T1D individuals that are insulin resistant, while there is no association with cardiovascular complications.

\section{1}

\section{Towards a GMP-Compliant Protocol for the Differentiation of Human Pluripotent Stem Cells to Beta-like Cells for the Treatment of Type 1 Diabetes}

\author{
Bahareh Rajaei ${ }^{1}$ Bas Brinkhof, Amadeo Muñoz García, Eelco de Koning, Françoise Carlotti ${ }^{1}$ \\ ${ }^{1}$ Department of Internal Medicine, Leiden University Medical Center, Leiden, The Netherlands
}

\section{BACKGROUND}

The generation of insulin-producing pancreatic $\beta$-cells from human pluripotent stem cells (hPSC) in vitro would provide an unlimited cell source for drug discovery and cell replacement therapy for diabetes. Here we aim to generate $\beta$-cells 
under GMP (Good Manufacturing Practice)-compliant conditions that can be used for clinical application.

\section{METHODS}

We applied a modified seven-stage (30-day) differentiation protocol to generate hPSC-derived insulin-producing $\beta$-like cells in a 3D microwell and scalable spinner flask culture system. $\beta$-cell function was evaluated in vitro by GSIS (Glucose-Stimulated Insulin Secretion), and in vivo by intraperitoneal glucose tolerance tests after transplantation of stage-7 cell clusters in immunodeficient mice and human C-peptide secretion was determined by ELISA. Single-cell RNA sequencing of stage 7 clusters was carried out. The expression profile of developmentally relevant genes and markers across the cell types was assessed.

\section{RESULTS}

In the microwell set-up, cells acquired a pancreatic progenitor phenotype at stage 4 (day 12), characterized by the co-expression of PDX1/NKX6.1 $(46.7 \% \pm 3.5 ; \mathrm{n}=3)$. At the end of stage 7 (day 30 ), C-peptide-positive $\beta$-like cells
$(49.5 \% \pm 10.5 ; \mathrm{n}=3)$ and glucagon-positive $\alpha$-like cells $(18.4 \% \pm 6.1 ; \mathrm{n}=3)$ were present. The stimulation index upon exposure to glucose was $4.4 \pm 3.1(\mathrm{n}=5)$. Following transplantation of day-30 clusters into mice, stimulated human C-peptide levels reached $57.2 \mathrm{pmol} / \mathrm{L}(\mathrm{n}=16), 80.5$ $\mathrm{pmol} / \mathrm{L}(\mathrm{n}=16)$ and $391.8 \mathrm{pmol} / \mathrm{L}(\mathrm{n}=9)$ at day 14,28 and 60 respectively, indicating further maturation of the cells in vivo. Comparable differentiation efficiency was obtained in disposable spinner flasks. Preliminary single-cell transcriptomics data allowed us to identify and characterize all cell types present in the stage 7 clusters.

\section{CONCLUSION}

hPSCs-derived islets are a promising future alternative to donor islets for the treatment of type 1 diabetes. We can generate functional hPSCs-derived $\beta$-like cells in GMP-compliant conditions in vitro. We are currently applying these protocols to a clinical-grade PSC line that we expanded and banked in the GMP facility of our institute.

\section{2}

\section{'I am my own doctor': a qualitative study of the perspectives and decision-making process of Muslims with diabetes on Ramadan fasting}

Siham Bouchareb ${ }^{1 *}$, Rabab Chrifou, Zohra Bourik, Giel Nijpels, Mohamed Hassanein, Marjan J. Westerman, Petra J.M. Elders ${ }^{1}$

${ }^{1}$ Department of General Practice, Amsterdam UMC (location VUmc), Amsterdam Public Health research institute, Amsterdam, The Netherlands

${ }^{*}$ Corresponding authors

E-mail: S.bouchareb@amsterdamumc.nl

\section{BACKGROUND}

Many Muslims with diabetes choose to fast against medical advice during Ramadan, potentially increasing their risk of acute complications. Patients are often reluctant to disclose fasting to their health care providers, and their needs regarding Ramadan are not met in consultations. For healthcare professionals to provide patient-centred care, it is important to gain more insight into patients' decision-making process. This study therefore aims to explore how Muslims with diabetes decide whether to fast during Ramadan.

\section{METHODS}

A qualitative study was conducted consisting of 15 focus groups with Muslims with diabetes. All focus groups were transcribed verbatim and analyzed using Braun and Clarke's thematic analysis.

\section{RESULTS}

Four themes were found to be important in the decision on whether to fast: (1) values and beliefs concerning Rama- dan, (2) experiences and emotions concerning Ramadan, (3) the perception of illness, and (4) advice from health care professionals, imams and family. Many participants indicated fasting against medical advice and trusting their subjective assessments on whether they could fast. Moreover, three main stages in the decision-making process for eventually refraining from fasting were identified: (1) the stage where positive experiences with fasting dominate, (2) the stage where one encounters challenges but their determination to fast prevails and (3) the stage where one decides to refrain from fasting after experiencing too many physical difficulties with fasting.

\section{CONCLUSION}

Muslims with diabetes experience autonomy in their decisions on Ramadan fasting. The decision to refrain from fasting often resulted from a difficult and dynamic decision-making process and was often made after participants reached their physical limits. These findings highlight the importance of not only shared decision-making to empower patients to make well-informed decisions on Ramadan fasting but also pre-Ramadan diabetes education to help patients fast safely. 


\title{
Methylprednisolone prevents insulin dysfunction and cell death in human islets exposed to proinflammatory cytokines
}

\author{
Miriam Paz Barba ${ }^{1}$, Françoise Carlotti ${ }^{1}$, Eelco de Koning ${ }^{1}$ \\ Nephrology, Leiden University Medical Centre, Leiden, The Netherlands \\ E-mail:M.Paz_Barba@lumc.nl
}

\section{BACKGROUND}

Methylprednisolone (MP), an immunosuppressive synthetic glucocorticoid that blocks immune cell activation, is widely used in autoimmune diseases like rheumatoid arthritis and more recently in severe COVID19 patients. MP is also given as immunosuppression treatment for type 1 diabetes (T1D) patients prior, during and after islet transplantation (3 days in total), in order to avoid organ rejection and inflammation. Even though the actions of corticosteroids have been associated to insulin resistance, we hypothesized that short-term methylprednisolone can have beneficial effects on human islets exposed to the inflammatory milieu which T1D patients are exposed in islet transplantation.

\section{METHODS}

Human pancreatic islets were incubated with low (L-MP $2,5 \mu \mathrm{M})$, high (H-MP $25 \mu \mathrm{M}$ ) or without (no-MP) methylprednisolone for $72 \mathrm{~h}$ in the presence of pro-inflammatory cytokines IFN $\gamma(50 \mathrm{ng} / \mathrm{ml})$ and IL- $1 \beta(1 \mathrm{ng} / \mathrm{ml})$. Islets were analysed for insulin secretion upon glucose stimuli (static GSIS), gene expression (qPCR) and cell viability (Caspase $3 / 7$ activity).

Preliminary data indicate that both low and high methylprednisolone-treated human islets exposed to cytokines for $72 \mathrm{~h}$ displayed an improved insulin secretion compared to cytokine control (Stimulation index of $1.5 \mathrm{x}, 2.5 \mathrm{x}$ and $6 \mathrm{x}$, for no-MP, L-MP and H-MP respectively). Cytokine-induced apoptosis was significantly decreased by both L-MP and H-MP treatments $(2.5 \mathrm{x}, 1.09 \mathrm{x}$ and $1.3 \mathrm{x}$, no-MP, L-MP and $\mathrm{H}-\mathrm{MP}$, respectively, $\mathrm{p}<0.05$ for both doses, $\mathrm{n}=3$ ). In addition, both methylprednisolone doses showed a trend towards reduced expression of pro-inflammatory cytokines such as IL-8 (Fold to control of $4.2 \mathrm{x}, 1.7 \mathrm{x}$ and $1 \mathrm{x}$, for no-MP, L-MP and H-MP respectively, $n=3$ ) and reduced ER-stress marker ATF3 (Fold to control of 2x, 1.7x and 1.1x, for no-MP, L-MP and H-MP respectively, $\mathrm{n}=2$ ). Moreover, we observed a trend towards blocking the cytokine-induced reduced insulin gene expression (Fold to control of $0.3 \mathrm{x}, 1 \mathrm{x}$ and $0.6 \mathrm{x}$ for no-MP, L-MP and H-MP respectively, $\mathrm{n}=3$ ).

\section{CONCLUSION}

Collectively our data indicate that methylprednisolone treatment inhibits cytokine-induced human beta-cell dysfunction and death, and it shows a trend towards decreasing pro-inflammatory and ER-stress related markers.

\section{4}

\section{The increase of the iron-transporting glycoprotein ceruloplasmin upon inflammatory stress is specific for the alpha cell population}

\author{
Fransje Boot ${ }^{1}$, Amadeo Muñoz Garcia ${ }^{1}$, Françoise Carlotti ${ }^{1}$, Eelco de Koning ${ }^{1}$ \\ ${ }^{1}$ Department of Internal Medicine, LUMC, Leiden, The Netherlands \\ E-mail:f.w.j.boot@lumc.nl
}

\section{BACKGROUND}

Type 1 diabetes is a beta-cell specific disease, leading us to the question what protective mechanisms alpha cells could have developed, that beta-cells do not have? Transcriptional characterization of pancreatic islet cells by single cell sequencing can help to define (sub)populations and individual gene. We analyzed data with a focus on alpha vs beta-cells after inflammation, looking for specific genes in the alpha cell population lacking in the beta cell population after this treatment.

\section{METHODS}

Intact human islets were incubated with pro-inflammatory conditions (IFN-y (50ng/mL) and IL-1b (1 ng/mL)) for $24 \mathrm{~h}$ and $72 \mathrm{~h}$ and analyzed by single-cell RNA sequencing. Alpha-TC cells (murine alpha-cell line) were incubated with pro-inflammatory conditions or the ER stressor Thapsigargin for $24 \mathrm{~h}$, and analyzed by qPCR and immunohistochemistry for glucagon and ceruloplasmin.

\section{RESULTS}

Single-cell transcriptomics data showed upregulation of ce- 
cCS bohn bohn
stafleu van loghum

SSS bohn

CL van loghum

SSS bohn

L van loghum

c) bohn stafleu

L van loghum

cer bohn stafleu

L van loghum

ceg bohn bohn
stafleu van loghum

c8h bohn stafleu van loghum

ç bohn stafleu van loghum

c) bohn stafleu van loghum

CS) bohn D) stafleu L. van loghum

cehn bohn stafleu van loghum
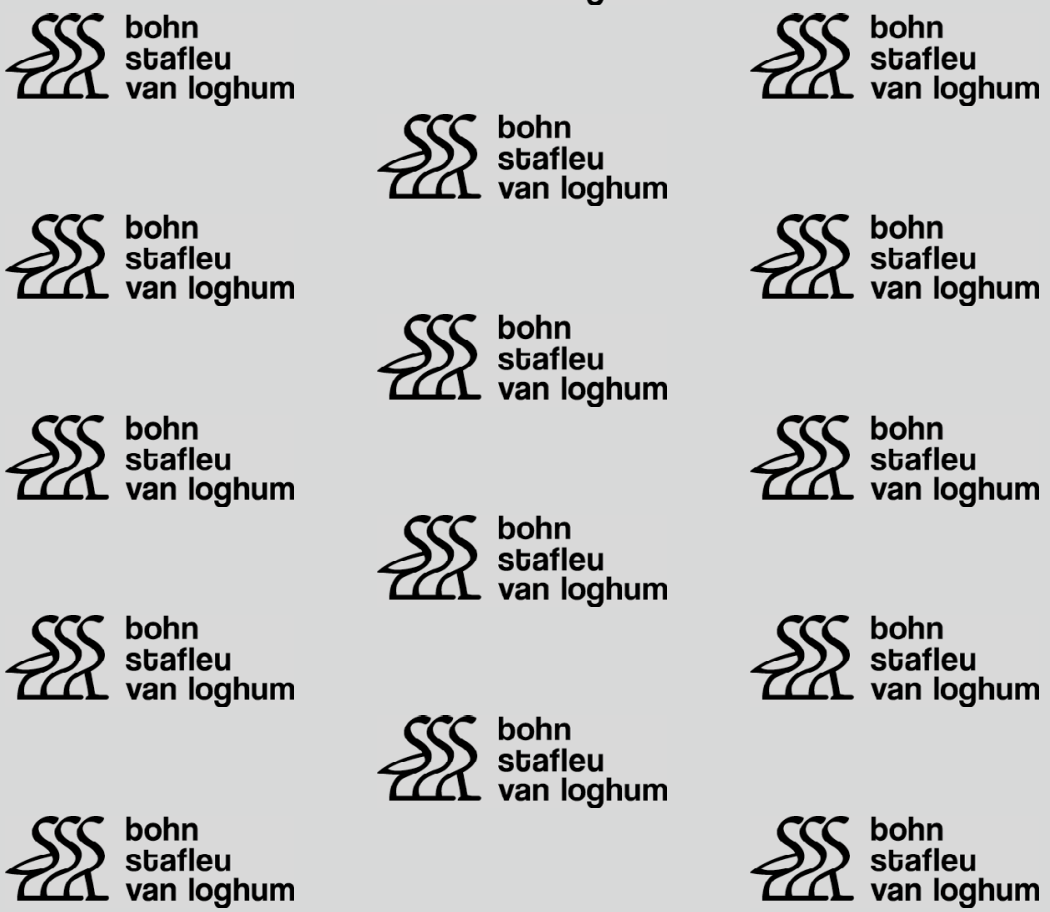

SS lohn

CI van loghum

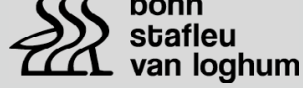

SSS bohn

SSS bohn

CSS bohn stafleu van loghum

SSS bohn stafleu van loghum

SSS bohn

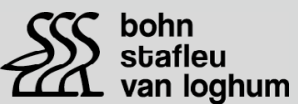

SSS bohn
SSS bohn stafleu van loghum
SSS lohn

SSS bohn

CL van loghum

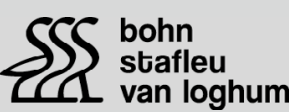

SSS bohn

CL van loghum

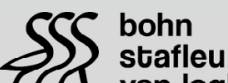

CL $\begin{aligned} & \text { stafleu } \\ & \text { van loghum }\end{aligned}$

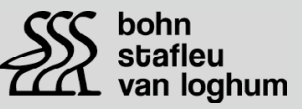

SSS bohn

IL van loghum

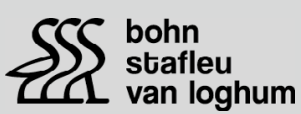

SSS bohn

CL van loghum

SSS lahn $\begin{aligned} & \text { bohn } \\ & \text { vanleu } \\ & \text { van loghum }\end{aligned}$

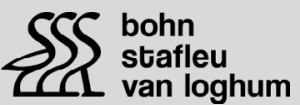

SSS bohn

CL van loghum bohn
stafleu Ll van loghum

SSS bohn II van loghum
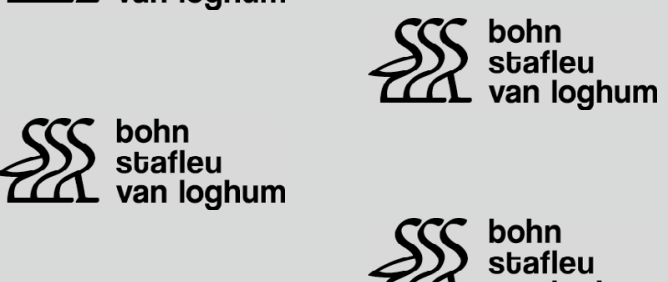
CL van loghum

CI van loghum

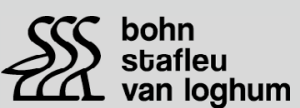

SSS bohn

Cl. van loghum
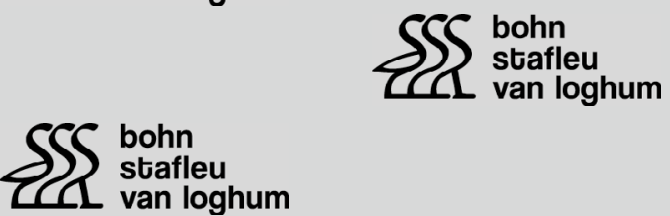

SSS bohn CL van loghum CL van loghum

SSS bohn

CL $\begin{aligned} & \text { stafleu } \\ & \text { van loghum }\end{aligned}$

SSL $\begin{aligned} & \text { bohn } \\ & \text { stafleu } \\ & \text { van loghum }\end{aligned}$

SSS bohn

CL van loghum

SSS bohn

CL van loghum

SSS bohn

CL van loghum

cSc bohn

$\sum$ stafleu

SSl bohn

van loghum

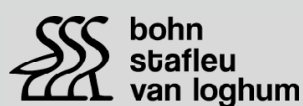

SCS bohn

$\sum \int$ stafleu

L van loghum

SSS bohn

CL van loghum

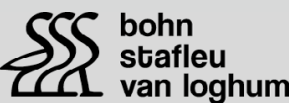

CL $\begin{aligned} & \text { stafleu } \\ & \text { van loghum }\end{aligned}$

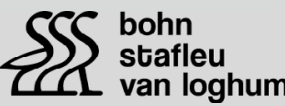

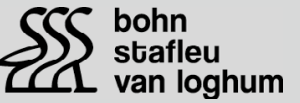
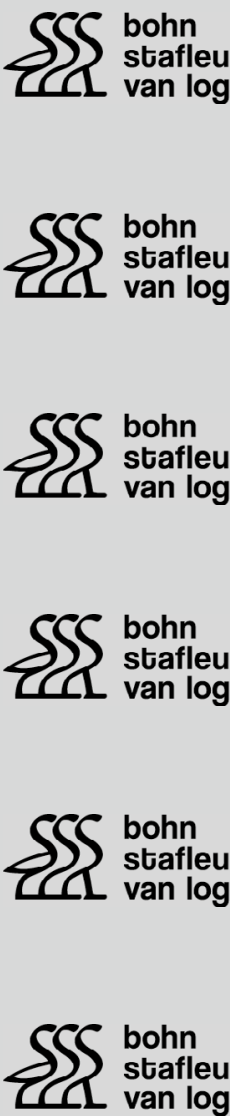

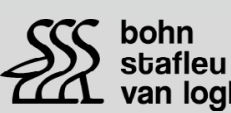

SSS $\begin{aligned} & \text { bohn } \\ & \text { stafleu } \\ & \text { van log }\end{aligned}$

SSS lohn

SSS bohn

ces bohn

CLL van log

L van log
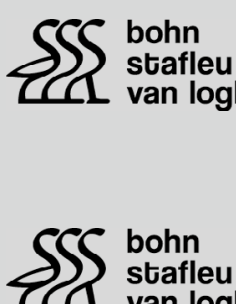

$3 \int \begin{aligned} & \text { bohn } \\ & \text { stafleu } \\ & \text { van log }\end{aligned}$

$\sum S \begin{aligned} & \text { bohn } \\ & \text { stafleu } \\ & \text { van log }\end{aligned}$ 
ruloplasmin (CP) after cytokine treatment in the alpha cells of human islets $(\mathrm{p}<0.0001, \mathrm{n}=3)$. Preliminary results show an increase for ceruloplasmin after cytokine or thapsigargin treatment in alpha cells on a gene expression level $(2 \mathrm{x}$ and $2.5 \mathrm{x}$ respectively) and protein level (1.6x for both).

\section{CONCLUSION}

Inflammation, as well as ER stress, show increased ceruloplasmin levels in alpha cells. Human beta cells are known to be susceptible for oxidative and ER stress in the development of diabetes, and a lack of ceruloplasmin production by the liver can cause dysregulation in insulin production, due to accumulation of iron in the cells. The increase of endogenous ceruloplasmin in the alpha cells, lacking in beta cells, possibly acts like an internal defense mechanism against ER and oxidative stress, helping the cells to function and survive during inflammation.

\section{5 \\ Clinical research for the usefulness of the Gastrointestinal Symptom Rating Scale (GSRS) to evaluate related side effects of metformin.}

O.W.F. Natan, R.J.A. Diepersloot, A.A.M. Franken, K.P. Bouter

\section{BACKGROUND}

Metformin is worldwide a first line prescribed drug for the treatment of Diabetes Mellitus type 2 (T2DM). Metformin is a proven effective and safe drug, but side effects and metformin intolerance occur. Intolerance to metformin is usually characterized by gastrointestinal (GI) side effects. Studies reported over 30\% GI side effects in patients starting with metformin therapy and 11.4-16.1\% discontinued their treatment within a year. ${ }^{1-2}$ Metformin intolerance has no official definition. L.J. McCreight defined metformin intolerance as those who had previously been treated with a maximum of $1000 \mathrm{mg}$ metformin daily for a maximum of 8 weeks, and discontinued treatment because of GI upset. Alternatively, intolerance was defined as inability to increase metformin dose above $500 \mathrm{mg}$ without experiencing GI side effects. ${ }^{3}$ The GSRS questionnaire is used for different indications like abdominal pain, irritable bowel, reflux, diarrhea and constipation syndrome and to compare influences of diets or medication on the gastrointestinal tract. ${ }^{4-6}$ We wanted to investigate the usefulness of the GSRS questionnaire in diabetic patients with side effects and possible intolerance for metformin.

\section{METHODS}

The PubMed database was screened using metformin, side effects, intolerance and GSRS as search items. In addition, references in the selected publications were screened for relevant studies on the subject. With the information from the literature, a clinical research was established. Diabetic patients, who are on metformin therapy (500-3000 mg/ day) were interviewed with the questions of the GSRS. Most of the included patients were already treated for a longer period based on titration. The GSRS questionnaire has 15 questions to evaluate side effects focused on: diarrhea, constipation, abdominal pain, digestion and reflux symptoms. The use of a score of 50 (105 total) as a standardized cut off point would enable researchers to compare the outcome of their clinical trials in patients with metformin intolerance.
Figure 3.

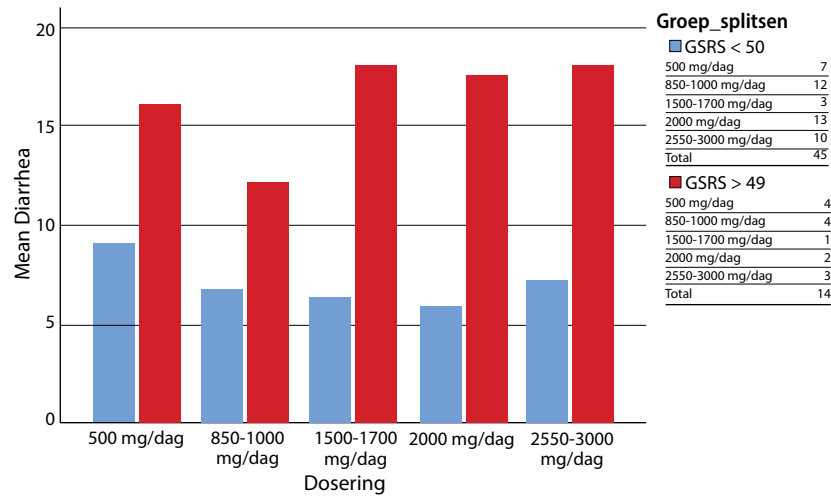

Figure 4.

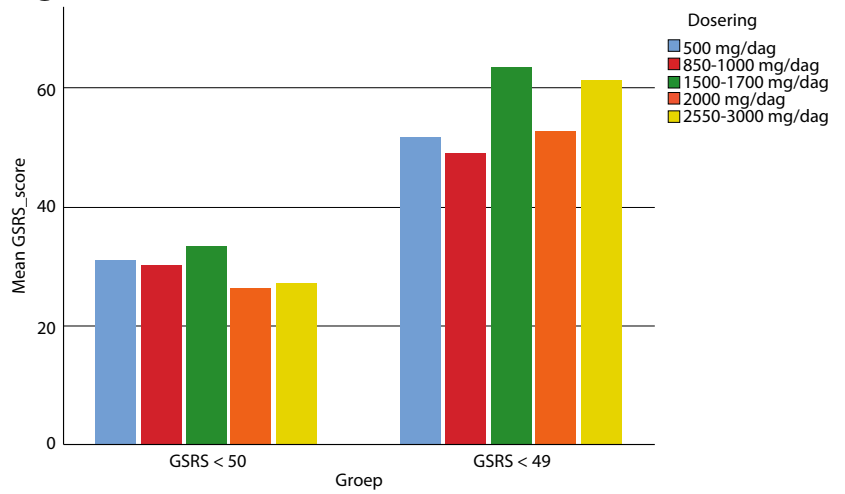

Table 5.

\begin{tabular}{lcc} 
Number of patients & Group 1; GSRS $<\mathbf{5 0}$ & Group 2; GSRS $>$ 49 \\
\hline $500 \mathrm{mg} / \mathrm{day}$ & 7 & 4 \\
\hline $850-1000 \mathrm{mg} / \mathrm{day}$ & 12 & 4 \\
\hline $1500-1700 \mathrm{mg} / \mathrm{day}$ & 3 & 1 \\
\hline $2000 \mathrm{mg} / \mathrm{day}$ & 13 & 2 \\
\hline $2550-3000 \mathrm{mg} /$ day & 10 & 3 \\
\hline
\end{tabular}

\section{RESULTS}

In this study 59 patients were interviewed ( 43 male, 16 female), with the mean age of 63 years old. During this 
study 2 groups were observed. In group 1. 45 patients with a GSRS score $<50$ and in group 2. 14 patients with a GSRS score $>49$. The most important GSRS scores were shown in figure 3 and 4 . Based on the cut-off $23,7 \%$ of the included patients should have an intolerance for metformin.

\section{CONCLUSION}

During this study, the GSRS questionnaire seems to be a good questionnaire to evaluate the most important side effects of metformin. A cut-off point of 50 gives a good overview of patients who are intolerant of metformin. With a score $>49$, it is recommendable to adjust the metformin treatment. For example: lowering the dose of metformin, switch to an extended-release metformin formulation or discuss alternative medicines. Despite most of the included patients are already titrated on the optimal dose versus side effects, patients seems to be loyal to the prescription from their doctor.

\section{REFERENCES}

1 De Jong L, Harmark L, Van Puijenbroek E, et al. Time course, outcome and management of adverse drug reactions associated with metformin from patient's perspective: a prospective, observational cohort study in the Netherlands. 2016, European Journal of Clinical Pharmacology, pp. 72(5): 615-622.

2 Plat A, Penning-van Beest F, Kessabi S, Groot M, et al. Change of initial oral antidiabetic therapy in type 2 diabetic patients. 2009, Pharmacy World and Science, pp. 31(6): 622-626.

3 McCreight LJ, Tore B, Connelly P, et al. Pharmacokinetics of metformin in patients with gastrointestinal intolerance. 2018, Diabetes, Obesity and Metabolism, pp. 20: 1593-1601.

4 Souza GS, Sarda FAH, Giuntini EB, et al. Translation and validation of the brazilian portuguese version of the gastrointestinal symptom rating scale (GSRS) questionnaire. 2016, Arquivos de Gastroenterologia, volume 53 no 3.

5 Kisler BM, Biruete A, Chapman-Novakofski K, et al. The relationship between intradialytic nutrition and gastrointestinal symptoms using a modified version of the gastrointestinal symptom rating scale. 2018, Journal of Renal Nutrition, volume 28 no 2, pp 129-134.

6 Halmos EP, Power VA, Shepherd SJ, et al. A diet low in FODMAPs reduces symptoms of irritable bowel syndrome. 2014, Gastroenterology, 146: 67-75.

\section{6}

\section{New perspectives in metformin intolerance.}

\section{K.P. Bouter, O.W.F. Natan, A.A.M. Franken, R.J.A. Diepersloot}

\section{BACKGROUND}

Because of metformin's safety profile, low cost, weight loss and cardiovascular benefits metformin has been included as the first line drug in several major guidelines on the treatment of Diabetes Mellitus type 2 and prediabetes. Intolerance to metformin is usually characterized by gastrointestinal (GI) adverse events. Studies reported over 30\% GI adverse events in patients starting with metformin therapy and 11.4-16.1\% discontinued their treatment within a year. $^{1-2}$

Aims: To investigate the prevalence and mechanism of metformin intolerance and to present alternative strategies in the treatment of patient with diabetes.

\section{METHODS}

The PubMed database was screened using metformin and intolerance as search items. In addition, references in the selected publications were screened for relevant studies on the subject

\section{RESULTS}

To prevent metformin adverse events, it is recommended to take the medication with a meal and to titrate the dose based on the occurrence of GI side effects. Doses above $2000 \mathrm{mg}$ per day should be avoided because they hardly lead to a further lowering of glucose. Occasionally, loperamide or cholestyramine is prescribed to prevent the adverse events. GI intolerance plays an important role in the decision to switch to other oral glucose-lowering drugs or to discontinue the treatment with metformin. GI intolerance to metformin remains difficult to understand and has been the subject of much scientific research. The results of these studies are sometimes confusing or contradictory: the prevalence of GI adverse events ranges from $0-60 \%$, complaints are linked to both lower prevalence of coronary heart disease and higher LDL cholesterol levels. In addition, elevated levels of Serotonin, Bile Acids, Lactate, and Glucagon-Like-Peptide-1 (GLP-1) have been suggested as a possible explanation without convincing clinical evidence. Recent research does come up with better substantiated explanations: reduced metformin transport and thus increased metformin concentrations in the gut is linked to reduced functionality alleles in OCT-1. Patients who carry two or more of these alleles are at increased risk for metformin intolerance. ${ }^{(3-5)}$ In addition, metformin has been shown to have an antibiotic effect on common bacterial pathogens and the microbiome, linked to GI intolerance. Clinical follow-up cohort studies suggest a better therapy compliance in patients that started on metformin extended release (MER). 


\section{CONCLUSION}

If a patient wishes to discontinue treatment due to metformin intolerance, the nocebo effect could be discussed with the patient and treatment resumed after a short interval. If this strategy is not successful, a trial treatment with MER would be warranted before switching to other glucose-lowering drugs.

\section{REFERENCES}

1 De Jong L, Harmark L, Van Puijenbroek E, et al. Time course, outcome and management of adverse drug reactions associated with metformin from patient's perspective: a prospective, observational cohort study in the Netherlands. 2016, European Journal of Clinical Pharmacology, pp. 72(5): 615-622.
2 Plat A, Penning-van Beest F, Kessabi S, Groot M, et al. Change of initial oral antidiabetic therapy in type 2 diabetic patients. 2009, Pharmacy World and Science, pp. 31(6): 622-626.

3 Dujic T, Zhou K, Donnelly LA, Roger Tavendale R, et al. Association of Organic Cation Transporter 1 With Intolerance to Metformin in Type 2 Diabetes: A GoDARTS Study. Diabetes. 2015, pp. 64(5): 1786-1793.

4 Stage TB, Lee MP, Hallas J, et al. Early discontinuation of metformin in individuals treated with inhibitors of transporters of metformin. Basic and Clinical Pharmacology and Toxicology. 2016, pp. 118: 487-495.

5 Dujic T, Causevic A, Bego T, Malenica M, et al. Organic cation transporter 1 variants and gastrointestinal side effects of metformin in patients with Type 2 diabetes. Diabetes Medicines. 2016, pp. 33(4): 511-514.

\title{
57 \\ Effect of lowering branched-chain amino acid levels in patients with type 2 diabetes using sodium-phenylbutyrate
}

\author{
F. Vanweert, E. Tapia, T. van de Weijer, J. Hoeks, V.B. Schrauwen-Hinderling, P. Schrauwen, M.K.C. Hesselink, M. Blair, M. Neinast, Z. Arany, E. Phielix \\ ${ }^{1}$ Department of Nutrition and Movement Sciences, NUTRIM School of Nutrition and Translational Research in Metabolism, Maastricht University, \\ Maastricht, The Netherlands
}

\section{BACKGROUND}

Patients with type 2 diabetes (T2D) are characterized by elevated branched-chain amino acid (BCAA) levels in plasma, which associate with insulin resistance. In the present study we aimed to pharmacologically accelerate BCAA oxidation in patients with T2D with use of sodium-phenylbutyrate $(\mathrm{NaPB})$ and evaluated the effect on patients' metabolic health.

\section{METHODS}

Sixteen men and women with T2D (BMI: $29.6 \pm 3.3 \mathrm{~kg} / \mathrm{m}^{2}$, age: $66 \pm 6 \mathrm{y}, 13 \mathrm{~m} / 3 \mathrm{f})$ underwent a 2-week NaPB $(4.8 \mathrm{~g} /$ $\mathrm{m}^{2}$ /day) treatment in a randomized, placebo-controlled, double-blind cross-over design with a wash-out period of 6-8 weeks. The primary outcome was peripheral insulin sensitivity, expressed as the change in insulin-stimulated glucose disposal rate minus baseline $(\Delta \mathrm{Rd})$. Secondary outcomes were metabolic flexibility, defined as the change in insulin-stimulated respiratory exchange ratio minus baseline $(\triangle \mathrm{RER})$, and ex vivo mitochondrial oxidative capacity in skeletal muscle expressed as $\mathrm{O}_{2}$-flux.

\section{RESULTS}

Fasting plasma BCAA levels (in $\mu \mathrm{mol} / \mathrm{l}$ ) decreased with $10 \%(\mathrm{p}=0.03)$ and fasting glucose levels tended to be lower
$(7.8 \pm 0.4$ vs. $8.2 \pm 0.5 \mathrm{mmol} / \mathrm{l}, \mathrm{p}=0.06)$ after $\mathrm{NaPB}$ treatment compared to placebo. Peripheral insulin-sensitivity increased with $27 \%$ ( $\Delta \mathrm{Rd}$ : $13.2 \pm 1.8$ vs. $9.6 \pm 1.8$ to $\mu \mathrm{mol} /$ $\mathrm{kg} / \mathrm{min}, \mathrm{p}=0.02$ ), primarily accounted for by a higher peripheral insulin-mediated glucose oxidation $(11.9 \pm 0.7$ vs. $10.8 \pm 0.6 \mu \mathrm{mol} / \mathrm{kg} / \mathrm{min}, \mathrm{p}=0.03$ ) with $\mathrm{NaPB}$ compared to placebo. Metabolic flexibility tended to be higher after $\mathrm{NaPB}$ compared to placebo $(\triangle \mathrm{RER}: 0.09 \pm 0.01$ vs. $0.08 \pm$ $0.01, \mathrm{p}=0.10$ ). Also, mitochondrial oxidation, fueled by the carbohydrate-derived substrate pyruvate, inceased $10 \%$ after $\mathrm{NaPB}$ compared to placebo (O2-flux: $74.0 \pm 4.1$ vs. $\left.67.1 \pm 4.3 \mathrm{pmol} /\left(\mathrm{s}^{\star} \mathrm{mg}\right), \mathrm{p}=0.05\right)$.

\section{CONCLUSION}

NaPB treatment for two weeks effectively reduced plasma BCAA levels in patients with $\mathrm{T} 2 \mathrm{D}$, paralleled by a higher peripheral insulin sensitivity and glucose oxidation. Our findings encourage future research to investigate the underlying mechanisms and whether stimulating BCAA oxidation could be a potential new treatment in $\mathrm{T} 2 \mathrm{D}$.

Clinical Trial Registration Number: NTR7426

Supported by: Diabetes Fonds Nederland 